\title{
On the Uplink Max-Min SINR of Cell-Free Massive MIMO Systems
}

\author{
Manijeh Bashar ${ }^{\circledR}$, Student Member, IEEE, Kanapathippillai Cumanan ${ }^{\circledR}$, Member, IEEE, \\ Alister G. Burr ${ }^{\circledR}$, Senior Member, IEEE, Mérouane Debbah, Fellow, IEEE, \\ and Hien Quoc $\mathrm{Ngo}^{\mathbb{1}}$, Member, IEEE
}

\begin{abstract}
A cell-free massive multiple-input multiple-output system is considered using a max-min approach to maximize the minimum user rate with per-user power constraints. First, an approximated uplink user rate is derived based on channel statistics. Then, the original max-min signal-to-interferenceplus-noise ratio problem is formulated for the optimization of receiver filter coefficients at a central processing unit and user power allocation. To solve this max-min non-convex problem, we decouple the original problem into two sub-problems, namely, receiver filter coefficient design and power allocation. The receiver filter coefficient design is formulated as a generalized Eigenvalue problem, whereas the geometric programming (GP) is used to solve the user power allocation problem. Based on these two sub-problems, an iterative algorithm is proposed, in which both problems are alternately solved while one of the design variables is fixed. This iterative algorithm obtains a globally optimum solution, whose optimality is proved through establishing an uplink-downlink duality. Moreover, we present a novel sub-optimal scheme which provides a GP formulation to efficiently and globally maximize the minimum uplink user rate. The numerical results demonstrate that the proposed scheme substantially outperforms the existing schemes in the literature.
\end{abstract}

Index Terms-Cell-free massive MIMO, max-min resource allocation, geometric programming, uplink-downlink duality, convex optimization, generalized eigenvalue problem.

Manuscript received November 20, 2017; revised May 11, 2018 and August 12, 2018; accepted December 17, 2018. The research described in this paper was supported in part by the European Horizon 2020 Programme under MCSA-ITN-2016-SPOTLIGHT (Grant 722788) and MSCA-RISE-2015-ATOM (Grant 690750), and in part through a University of York scholarship. This work was also supported in part by the European Commission under the 5GPPP project 5GXcast (H2020-ICT-2016-2 call, grant number 761498). The views expressed in this contribution are those of the authors and do not necessarily represent the project. The associate editor coordinating the review of this paper and approving it for publication was B. Natarajan. (Corresponding author: Manijeh Bashar.)

M. Bashar is with the Department of Electronic Engineering, University of York, York YO10 5DD, U.K., and also with the 5G Innovation Centre, Institute for Communication Systems, University of Surrey, Guildford GU2 7XH, U.K. (e-mail: mb1465@york.uc.ak; m.bashar@surrey.ac.uk).

K. Cumanan and A. G. Burr are with the Department of Electronic Engineering, University of York, York YO10 5DD, U.K. (e-mail: kanapathippillai.cumanan@york.ac.uk; alister.burr@york.ac.uk).

M. Debbah is with the Large Networks and Systems Group, CentraleSupelec, Universite Paris-Saclay, 91192 Gif-sur-Yvette, France, and also with the Mathematical and Algorithmic Sciences Lab, Huawei Technologies Co., Ltd., 92100 Boulogne-Billancourt, France (e-mail: merouane.debbah@ centralesupelec.fr).

H. Q. Ngo is with the School of Electronics, Electrical Engineering and Computer Science, Queen's University Belfast, Belfast BT3 9DT, U.K. (e-mail: hien.ngo@qub.ac.uk).

Color versions of one or more of the figures in this paper are available online at http://ieeexplore.ieee.org.

Digital Object Identifier 10.1109/TWC.2019.2892463

\section{INTRODUCTION}

$\mathbf{F}$ UTURE fifth generation (5G) wireless communication networks will deliver a wide range of new user services and dramatically increased data rates. Massive multiple-input multiple-output (MIMO) has been recognized as one of the key elements of $5 \mathrm{G}$ systems, due to its potential for extremely high spectral efficiency [1]-[3]. This paper considers cell-free Massive MIMO which has received much attention recently because of its potential to ensure uniformly good service throughput for all users [4]-[8]. Cell-free Massive MIMO is a combination of distributed MIMO and Massive MIMO, and there is no cell boundary [4]. It is a scalable version of network MIMO which is also called coordinated multipoint processing (CoMP) [9], [10]. The distributed access points (APs) are connected to a central processing unit (CPU) via high capacity backhaul links [4]. Cell-free Massive MIMO is thus also a scalable version of the cloud radio access network (CRAN). In CRAN, there are heavy communication burdens on the backhaul, and computation burdens on the CPU, as all signal processing is performed at the CPU [11]. The fog radio access network (FRAN) [12] can overcome some of the problems of CRAN. It moves some signal processing functionalities from the CPU back to the AP, where in this case the APs can also perform part of the signal processing. Hence, the tasks required of the CPU can also be reduced. The more processing is moved to the AP, the less is the burden imposed on the CPU.

In [4], [6], and [13], the authors propose that the APs design the linear receivers based on the estimated channels, and that this is carried out locally at the APs. Hence, the CPU exploits only the statistics of the channel for data detection. However, in this paper, we propose to exploit a new receiver filter at the CPU to improve the performance of cell-free Massive MIMO systems. The coefficients of the proposed receiver filter are designed based on only the statistics of the channel, which is different from the linear receiver at the APs. The proposed receiver filter provides more freedom in the design parameters, and hence significantly improves the performance of the uplink of cell-free Massive MIMO. In other words, the receiver filter coefficients are designed after exploiting linear detection at the CPU. Therefore, the uplink problem in the present paper is different from the problem studied in [4], as in [4], the authors do not consider the receiver filter coefficients. 
In this paper, we investigate an uplink max-min signalto-interference-plus-noise ratio (SINR) problem in a cell-free Massive MIMO system. In particular, we propose a new approach to solve this max-min problem. A similar max-min SINR problem based on SINR known as SINR balancing in the literature has been considered for cognitive radio networks in [14]-[16]. In [17] and [18], the authors consider MIMO systems and study the problem of max-min user SINR to maximize the smallest user SINR. Note that the same maxmin problem is investigated in an uplink cell-free Massive MIMO systems in [4] where user power allocation is utilized by using a bisection search approach. However, the maxmin SINR problem considered in this paper is different from the scheme in [4] due to the design parameters (in terms of receiver filter coefficients and user power allocation) and solution approach. In particular, the receiver filter coefficients and power allocation are optimized in the proposed approach whereas the work in [4] only considered user power allocations. First, we derive the average SINR of the user by incorporating a matched filtering receiver and formulate the corresponding max-min SINR problem. This original maxmin problem in terms of receiver filter coefficients and power allocations is not jointly convex. To circumvent this nonconvexity issue, we decompose the original problem into two sub-problems, namely, receiver filter coefficient design, and power allocation. It is shown that the receiver filter coefficient design problem can be solved through a generalized eigenvalue problem [19] whereas the user power allocation problems can be formulated using standard geometric programming (GP) [20], [21]. An iterative procedure is proposed whereby at each iteration, one of the sub-problems is solved while the other design variable is fixed. To validate the optimality of the proposed scheme, we show that there exists an equivalent downlink problem to realize the same user rate in the uplink with an equivalent total power constraint and the same receiver filter coefficients. By solving this equivalent problem, the optimality of the proposed scheme in the uplink is proved. The problem of uplink-downlink duality has been investigated in [17] and [22]-[25]. Simulation results are provided to demonstrate the performance of the proposed scheme which confirms that the proposed scheme outperforms the scheme in [4] in terms of achieved user rate. In addition, we propose a new sub-optimal max-min SINR scheme using a GP formulation which does not require any iterative approach as in [4]. The contributions and results are as follows:

1. To improve the performance of the system, we propose to use a novel receiver filter, operating at the CPU, which can be designed based only on the statistics of the channel. Note that this is different from the linear matched filtering receiver in [4].

2. The uplink user throughput using the proposed filter is derived based on channel statistics and taking into account the effects of channel estimation errors and the effect of pilot sequences. We propose a novel approach to solve the uplink max-min SINR problem, decoupling the original problem into two sub-problems, which are solved using an iterative algorithm. These sub-problems are

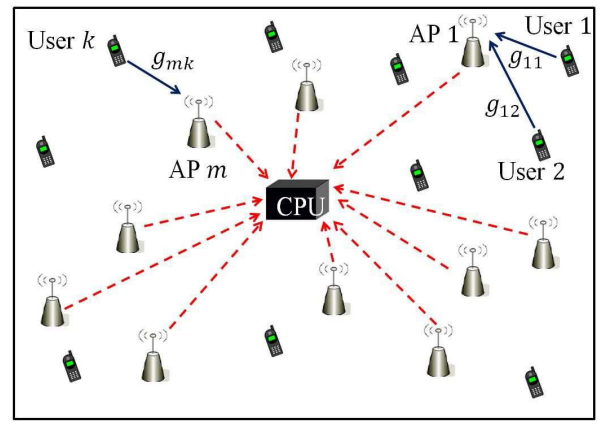

Fig. 1. The uplink of a cell-free Massive MIMO system with $K$ singleantenna users and $M$ APs. The solid lines denote the uplink channels and the dashed lines present the backhaul links from the APs to the CPU.

formulated as GP and a generalized eigenvalue problem, and both sub-problems are solved at each iteration.

3. We prove that the proposed iterative algorithm provides the globally optimal solution for the original non-convex max-min SINR problem. The optimality of the proposed algorithm is proved through establishing the uplinkdownlink duality for cell-free Massive MIMO.

4. We present a sub-optimal max-min SINR scheme by formulating it into a standard GP which does not require an iterative approach and shows the same performance as in [4].

5. We present the complexity analysis of different schemes.

6. We present numerical results supporting the convergence analysis and the theoretical derivations of the optimality of the proposed schemes.

\section{A. Outline}

The rest of the paper is organized as follows. Section II describes the system model, and Section III provides performance analysis. The proposed max-min SINR scheme is presented in Section IV and the convergence analysis is provided in Section V. The optimality of the proposed scheme is proved in Section VI. Section VII investigates a sub-optimal max-min SINR scheme. Complexity analysis and a proposed user assignment scheme are presented in Section VIII and Section IX, respectively. Finally, Section X provides numerical results while Section XI concludes the paper.

\section{B. Notation}

The following notations are adopted in the rest of the paper. Uppercase and lowercase boldface letters are used for matrices and vectors, respectively. The notation $\mathbb{E}\{\cdot\}$ denotes expectation. $|\cdot|$ stands for absolute value. The conjugate transpose of vector $\mathbf{x}$ is $\mathbf{x}^{H}$, and $\mathbf{X}^{T}$ denotes the transpose of matrix $\mathbf{X}$. In addition, $x \sim \mathcal{C N}\left(0, \sigma^{2}\right)$ represents a zeromean circularly symmetric complex Gaussian random variable with variance $\sigma^{2}$.

\section{System Model}

We consider uplink transmission in a cell-free Massive MIMO system with $M$ single-antenna APs and $K$ randomly distributed single-antenna users in the area, as shown in Fig. 1. 
The channel coefficient between the $k$ th user and the $m$ th AP, $g_{m k}$, is modeled as [4]

$$
g_{m k}=\sqrt{\beta_{m k}} h_{m k},
$$

where $\beta_{m k}$ denotes the large-scale fading and $h_{m k} \sim$ $\mathcal{C N}(0,1)$ represents small-scale fading between the $k$ th user and the $m$ th AP.

\section{A. Uplink Channel Estimation}

In order to estimate channel coefficients in the uplink, the APs employ an minimum mean-square error (MMSE) estimator. During the training phase, all $K$ users simultaneously transmit their pilot sequences of length $\tau$ symbols to the APs. Let $\sqrt{\tau} \phi_{k} \in C^{\tau \times 1}$, where $\left\|\phi_{k}\right\|^{2}=1$, be the pilot sequence assigned to the $k$ th user. Then, the received signal at the $m$ th AP is given by

$$
\mathbf{y}_{m}^{p}=\sqrt{\tau p_{p}} \sum_{k=1}^{K} g_{m k} \boldsymbol{\phi}_{k}+\mathbf{w}_{m}^{p},
$$

where vector $\mathbf{w}_{m}^{p} \in \mathbb{C}^{\tau \times 1}$ is the noise whose elements are i.i.d $\mathcal{C N}(0,1)$. Next, the APs exploit the pilot sequence $\phi_{k}$ to correlate the received signal with the pilot sequence as follows [4]:

$$
\check{\mathbf{y}}_{m, k}^{p}=\phi_{k}^{H} \mathbf{y}_{m}^{p}=\sqrt{\tau p_{p}} g_{m k}+\sqrt{\tau p_{p}} \sum_{k^{\prime} \neq k}^{K} g_{m k^{\prime}} \boldsymbol{\phi}_{k}^{H} \phi_{k^{\prime}}+\dot{w}_{m k}^{p},
$$

where $\dot{w}_{m k}^{p} \triangleq \phi_{k}^{H} \mathbf{w}_{m}^{p}$. The linear MMSE estimate of $g_{m k}$ is

$$
\begin{aligned}
\hat{g}_{m k} & =\frac{\mathbb{E}\left\{g_{m k} \check{\mathbf{y}}_{m, k}^{p}\right\}}{\mathbb{E}\left\{\left|\check{\mathbf{y}}_{m, k}^{p}\right|^{2}\right\}} \check{\mathbf{y}}_{m, k}^{p} \\
& =c_{m k}\left(\sqrt{\tau p_{p}} g_{m k}+\sqrt{\tau p_{p}} \sum_{k^{\prime} \neq k}^{K} g_{m k^{\prime}} \phi_{k}^{H} \boldsymbol{\phi}_{k^{\prime}}+\dot{w}_{m k}^{p}\right),
\end{aligned}
$$

where $c_{m k}$ is obtained as [4]

$$
c_{m k}=\frac{\sqrt{\tau p_{p}} \beta_{m k}}{\tau p_{p} \sum_{k^{\prime}=1}^{K} \beta_{m k^{\prime}}\left|\phi_{k}^{H} \boldsymbol{\phi}_{k^{\prime}}\right|^{2}+1} .
$$

Note that, as in [4], we assume that the large-scale fading, $\beta_{m k}$, is known. The estimated channels in (3) are used by the APs to design the receiver filter coefficients and determine power allocations at users to maximize the minimum rate of the users. In this paper, we investigate the cases of both random pilot assignment and orthogonal pilots in cell-free Massive MIMO. Here the term "orthogonal pilots" refers to the case where unique orthogonal pilots are assigned to all users, while in "random pilot assignment" each user is randomly assigned a pilot sequence from a set of orthogonal sequences of length $\tau(<K)$, following the approach of [4] and [26].

\section{B. Uplink Transmission}

In this subsection, we consider the uplink data transmission, where all users send their signals to the APs. The transmitted signal from the $k$ th user is represented by

$$
x_{k}=\sqrt{\rho q_{k}} s_{k},
$$

where $s_{k}\left(\mathbb{E}\left\{\left|s_{k}\right|^{2}\right\}=1\right)$ and $q_{k}$ denote the transmitted symbol and the transmit power from the $k$ th user, respectively. Moreover, $\rho$ refers to the normalized uplink SNR. The received signal at the $m$ th AP from all users is given by

$$
y_{m}=\sqrt{\rho} \sum_{k=1}^{K} g_{m k} \sqrt{q_{k}} s_{k}+n_{m},
$$

where $n_{m} \sim \mathcal{C N}(0,1)$ is the noise at the $m$ th AP. In addition, a matched filtering approach is employed at the APs, in that the received signal is weighted appropriately. More precisely, the received signal at the $m$ th AP, $y_{m}$, is first multiplied by $\hat{g}_{m k}^{*}$. The resulting $\hat{g}_{m k}^{*} y_{m}$ is then forwarded to the CPU for signal detection. In order to improve achievable rate, the forwarded signal is further multiplied by a receiver filter coefficient at the CPU. The aggregated received signal at the CPU can be written as

$$
\begin{aligned}
r_{k} & =\sum_{m=1}^{M} u_{m k} \hat{g}_{m k}^{*} y_{m} \\
& =\sqrt{\rho} \sum_{k^{\prime}=1}^{K} \sum_{m=1}^{M} u_{m k} \hat{g}_{m k}^{*} g_{m k^{\prime}} \sqrt{q_{k^{\prime}}} s_{k^{\prime}}+\sum_{m=1}^{M} u_{m k} \hat{g}_{m k}^{*} n_{m} .
\end{aligned}
$$

By collecting all the coefficients $u_{m k}, \forall m$ corresponding to the $k$ th user, we define $\mathbf{u}_{k}=\left[u_{1 k}, u_{2 k}, \cdots, u_{M k}\right]^{T}$ and without loss of generality, it is assumed that $\left\|\mathbf{u}_{k}\right\|=1$. The optimal solution for $\mathbf{u}_{k}, q_{k}, \forall k$ for the considered maxmin SINR approach is investigated in Section IV. Similar to [4], [6], and [13], we assume that the APs are connected to the CPU via perfect backhaul connections. Such perfect backhaul links might be established through fiber links between the APs and the CPU. Moreover, based on [27], copperbased backhaul links can provide a capacity of $750 \mathrm{Mbits} / \mathrm{s}$ for a maximum distance of $1.5 \mathrm{~km}$ between the APs and the CPU. In [28]-[30], the authors show that exploiting optimal uniform quantization and wireless microwave links with capacity $100 \mathrm{Mbits} / \mathrm{s}$ [31], the performance of limitedbackhaul cell-free Massive MIMO system closely approaches the performance of cell-free Massive MIMO with perfect backhaul links.

\section{iII. Performance Analysis}

In this section, we derive the achievable rate for the considered system model by following a similar approach to [4]. Note that the main difference between the proposed approach and the scheme in [4] is the new set of receiver filter coefficients which are introduced at the CPU to improve the achievable user rate. The benefits of the proposed approach in terms of the achievable uplink rate are demonstrated by the numerical results in Section V. In deriving the achievable rate of each user, it is assumed that the CPU exploits only the knowledge 
of channel statistics between the users and APs in detecting data from the received signal in (7). Without loss of generality, the aggregate received signal in (7) can be written as

$$
\begin{aligned}
& =\underbrace{\sqrt{\rho \mathbb{E}}\left\{\sum_{m=1}^{M} u_{m k} \hat{g}_{m k}^{*} g_{m k} \sqrt{q_{k}}\right\}}_{\mathrm{DS}_{k}} s_{k} \\
& +\underbrace{\sqrt{\rho}\left(\sum_{m=1}^{M} u_{m k} \hat{g}_{m k}^{*} g_{m k} \sqrt{q_{k}}-\mathbb{E}\left\{\sum_{m=1}^{M} u_{m k} \hat{g}_{m k}^{*} g_{m k} \sqrt{q_{k}}\right\}\right)}_{\mathrm{BU}_{k}} s_{k} \\
& +\sum_{k^{\prime} \neq k}^{K} \underbrace{\sqrt{\rho} \sum_{m=1}^{M} u_{m k} \hat{g}_{m k}^{*} g_{m k^{\prime}} \sqrt{q_{k^{\prime}}}}_{\mathbf{I U}_{k k^{\prime}}} s_{k^{\prime}}+\underbrace{\sum_{m=1}^{M} u_{m k} \hat{g}_{m k}^{*} n_{m}}_{\mathrm{TN}_{k}},
\end{aligned}
$$

where $\mathrm{DS}_{k}$ and $\mathrm{BU}_{k}$ denote the desired signal (DS) and beamforming uncertainty (BU) for the $k$ th user, respectively, and $\mathrm{IUI}_{k k^{\prime}}$ represents the inter-user-interference (IUI) caused by the $k^{\prime}$ th user. In addition, $\mathrm{TN}_{k}$ accounts for the total noise (TN) following the matched filtering. The corresponding SINR of the received signal in (8) can be defined by considering the worst-case of the uncorrelated Gaussian noise as follows [4]:

$$
\operatorname{SINR}_{k}^{\mathrm{UP}}=\frac{\left|\mathrm{DS}_{k}\right|^{2}}{\mathbb{E}\left\{\left|\mathrm{BU}_{k}\right|^{2}\right\}+\sum_{k^{\prime} \neq k}^{K} \mathbb{E}\left\{\left|\mathrm{IUI}_{k k^{\prime}}\right|^{2}\right\}+\mathbb{E}\left\{\left|\mathrm{TN}_{k}\right|^{2}\right\}}
$$

Based on the SINR definition in (9), the achievable uplink rate of the $k$ th user is defined in the following theorem:

Theorem 1: By employing the matched filtering approach at the APs, the achievable uplink rate of the kth user in the cell-free Massive MIMO system with $K$ randomly distributed single-antenna users and $M$ single-antenna APs is given by (10), shown at the bottom of this page. Note that in (10), we have

$$
\begin{aligned}
\boldsymbol{\Gamma}_{k} & =\left[\gamma_{1 k}, \gamma_{2 k}, \cdots, \gamma_{M k}\right]^{T} \\
\boldsymbol{u}_{k} & =\left[u_{1 k}, u_{2 k}, \cdots, u_{M k}\right]^{T}, \\
\boldsymbol{\Delta}_{k k^{\prime}} & =\left[\frac{\gamma_{1 k} \beta_{1 k^{\prime}}}{\beta_{1 k}}, \frac{\gamma_{2 k} \beta_{2 k^{\prime}}}{\beta_{2 k}}, \cdots, \frac{\gamma_{M k} \beta_{M k^{\prime}}}{\beta_{M k}}\right]^{T}, \\
\boldsymbol{R}_{k} & =\operatorname{diag}\left[\gamma_{1 k}, \gamma_{2 k}, \cdots, \gamma_{M k}\right], \\
\boldsymbol{D}_{k k^{\prime}} & =\operatorname{diag}\left[\beta_{1 k^{\prime}} \gamma_{1 k}, \beta_{2 k^{\prime}} \gamma_{2 k}, \cdots, \beta_{M k^{\prime}} \gamma_{M k}\right] .
\end{aligned}
$$

Proof: Please refer to Appendix A.

Note that the achievable rate in (10) is a function of only large-scale fading which changes less often than the actual channel. Hence, the rate formula and accordingly the power coefficients only need to be calculated when the large-scale fading changes. Therefore, the APs do not need frequently to update the CPU with the instantaneous channel state and the user rates will change only when the positions of the users change. Moreover, in cell-free Massive MIMO, due to the channel hardening property, detection using only the channel statistics is nearly optimal [4].

\section{Proposed MaX-Min SINR Scheme}

In this section, we formulate the max-min user-fairness problem in the cell-free Massive MIMO, where the minimum uplink rates of all users is maximized while satisfying the per-user power constraint. This max-min rate problem can be formulated as the following optimization framework:

$$
\begin{aligned}
P_{1}: & \max _{q_{k}, \mathbf{u}_{k}} \min _{k=1, \cdots, K} R_{k}^{\mathrm{UP},} \\
\text { s.t. } & \left\|\mathbf{u}_{k}\right\|=1, \quad \forall k, \\
& 0 \leq q_{k} \leq p_{\max }^{(k)}, \quad \forall k,
\end{aligned}
$$

where $p_{\max }^{(k)}$ is the maximum transmit power available at user $k$. From (10), it can be observed that in the denominator of the expression for the uplink SINR, the power coefficients $q_{k^{\prime}}, k^{\prime} \neq k$ are coupled with the receiver filter $\mathbf{u}_{k}$. Therefore, it is not possible to define a new variable $\mathbf{w}_{k}=$ $\sqrt{q_{k}} \mathbf{u}_{k}$, and solve the problem jointly in terms of $\mathbf{u}_{k}$ and $q_{k}$. As a result, Problem $P_{1}$ is not jointly convex in terms of $\mathbf{u}_{k}$ and power allocation $q_{k}, \forall k$. Therefore, this problem cannot be directly solved through existing convex optimization software. To tackle this non-convexity issue, we decouple the original problem $P_{1}$ into two sub-problems: receiver filter coefficient design (i.e., $\mathbf{u}_{k}$ ) and the power allocation problem. To obtain a solution for Problem $P_{1}$, these sub-problems are alternately solved as explained in the following subsections.

\section{A. Receiver Filter Coefficient Design}

In this subsection, we solve the receiver coefficient design problem to maximize the uplink rate of each user for a given set of transmit power allocations at all users. By following the analysis in [17], [22], and [23], the receiver filter coefficients (i.e., $\mathbf{u}_{k}, \forall k$ ) can be obtained by independently maximizing the uplink SINR of each user. Therefore, the optimal receiver filter coefficients for all users for a given set of transmit power allocations can be determined by solving the following optimization problem (13a) and (13b), shown at the bottom of this page.

$$
\begin{gathered}
R_{k}^{\mathrm{UP}}=\log _{2}\left(1+\frac{\mathbf{u}_{k}^{H}\left(q_{k} \boldsymbol{\Gamma}_{k} \boldsymbol{\Gamma}_{k}^{H}\right) \mathbf{u}_{k}}{\mathbf{u}_{k}^{H}\left(\sum_{k^{\prime} \neq k}^{K} q_{k^{\prime}}\left|\boldsymbol{\phi}_{k}^{H} \boldsymbol{\phi}_{k^{\prime}}\right|^{2} \boldsymbol{\Delta}_{k k^{\prime}} \boldsymbol{\Delta}_{k k^{\prime}}^{H}+\sum_{k^{\prime}=1}^{K} q_{k^{\prime}} \mathbf{D}_{k k^{\prime}}+\frac{1}{\rho} \mathbf{R}_{k}\right) \mathbf{u}_{k}}\right) . \\
P_{2}: \max _{\mathbf{u}_{k}} \frac{\mathbf{u}_{k}^{H}\left(q_{k} \boldsymbol{\Gamma}_{k} \boldsymbol{\Gamma}_{k}^{H}\right) \mathbf{u}_{k}}{\mathbf{u}_{k}^{H}\left(\sum_{k^{\prime} \neq k}^{K} q_{k^{\prime}}\left|\boldsymbol{\phi}_{k}^{H} \boldsymbol{\phi}_{k^{\prime}}\right|^{2} \boldsymbol{\Delta}_{k k^{\prime}} \boldsymbol{\Delta}_{k k^{\prime}}^{H}+\sum_{k^{\prime}=1}^{K} q_{k^{\prime}} \mathbf{D}_{k k^{\prime}}+\frac{1}{\rho} \mathbf{R}_{k}\right) \mathbf{u}_{k}} \\
\text { s.t. }\left\|\mathbf{u}_{k}\right\|=1, \quad \forall k .
\end{gathered}
$$




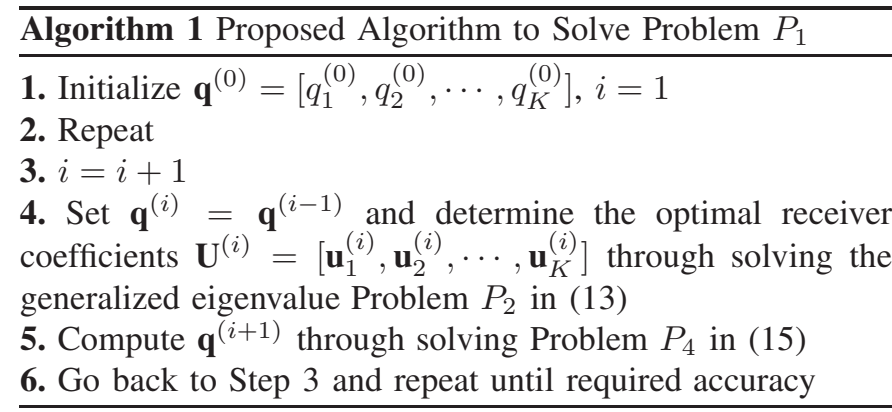

Problem $P_{2}$ is a generalized eigenvalue problem [19], where the optimal solutions can be obtained by determining the generalized eigenvalue of the matrix pair $\mathbf{A}_{k}=q_{k} \boldsymbol{\Gamma}_{k} \boldsymbol{\Gamma}_{k}^{H}$ and $\mathbf{B}_{k}=\sum_{k^{\prime} \neq k}^{K} q_{k^{\prime}}\left|\boldsymbol{\phi}_{k}^{H} \boldsymbol{\phi}_{k^{\prime}}\right|^{2} \boldsymbol{\Delta}_{k k^{\prime}} \boldsymbol{\Delta}_{k k^{\prime}}^{H}+\sum_{k^{\prime}=1}^{K} q_{k^{\prime}} \mathbf{D}_{k k^{\prime}}+\frac{1}{\rho} \mathbf{R}_{k}$ corresponding to the maximum generalized eigenvalue.

\section{B. Power Allocation}

In this subsection, we solve the power allocation problem for a given set of fixed receiver filter coefficients which can be formulated as the following max-min problem:

$$
\begin{gathered}
P_{3}: \max _{q_{k}} \min _{k=1, \cdots, K} \operatorname{SINR}_{k}^{\mathrm{UP}}, \\
\text { s.t. } 0 \leq q_{k} \leq p_{\max }^{(k)} .
\end{gathered}
$$

Without loss of generality, Problem $P_{3}$ can be rewritten by introducing a new slack variable as

$$
\begin{aligned}
P_{4}: \quad \max _{t, q_{k}} t, & \\
\text { s.t. } & 0 \leq q_{k} \leq p_{\max }^{(k)}, \quad \forall k, \\
& \operatorname{SINR}_{k}^{\mathrm{UP}} \geq t, \quad \forall k .
\end{aligned}
$$

Proposition 1: Problem $P_{4}$ can be formulated into a standard GP.

Proof: Please refer to Appendix B.

Therefore, this problem can be efficiently solved through existing convex optimization software. Based on these two sub-problems, an iterative algorithm is developed by alternately solving each sub-problem at each iteration. The proposed algorithm is summarized in Algorithm 1.

\section{CONVERGEnCE ANALYSis}

In this section, the convergence analysis of the proposed Algorithm 1 is provided. Two sub-problems are alternately solved to determine the solution to Problem $P_{1}$. At each iteration, one of the design parameters is determined by solving the corresponding sub-problem while other design variable is fixed. Note that each sub-problem provides an optimal solution for the other given design variable. At the $i$ th iteration, the receiver filter coefficients $\mathbf{u}_{k}^{(i)}, \forall k$ are determined for a given power allocation $\mathbf{q}^{(i)}$ and similarly, the power allocation $\mathbf{q}^{(i+1)}$ is updated for a given set of receiver filter coefficients $\mathbf{u}_{k}^{(i)}, \forall k$. The optimal power allocation $\mathbf{q}^{(i+1)}$ obtained for a given $\mathbf{u}_{k}^{(i)}$ achieves an uplink rate greater than or equal to that of the previous iteration. In addition, the power allocation $\mathbf{q}^{(i)}$ is also a feasible solution in determining $\mathbf{q}^{(i+1)}$ as the receiver filter coefficients $\mathbf{u}_{k}^{(i+1)}, \forall k$ are determined for a given $\mathbf{q}^{(i)}$. This reveals that the achieved uplink rate monotonically increases with each iteration, which can be also observed from the simulation results presented in Figs. 8 and 9. As the achievable uplink max-min rate is upper bounded by a certain value for a given set of per-user power constraints, the proposed algorithm converges to a particular solution. Fortunately, the proposed Algorithm 1 converges to the optimal solution, as we will prove by establishing the uplink-downlink duality in the following section.

\section{Optimality of the Proposed MAX-MIN SINR ALGORITHM}

In this section, we prove the optimality of the proposed maxmin SINR scheme in Algorithm 1. In general, converting the original non-convex problem into two sub-problems would remove the global optimality. However, the global optimality of the proposed Algorithm 1 can be proved as follows: first, we show that the solution of the original max-min Problem $P_{1}$ can be obtained by solving an uplink problem with an equivalent total power constraint instead of the peruser power constraint. Then, an uplink-downlink duality is established by proving that the same SINRs can be achieved in both the uplink and the downlink with an equivalent total power constraint. In other words, the same SINRs in the uplink Problem $P_{1}$ can be realized by solving an equivalent downlink problem. Finally, we present a bisection approach to determine the optimal solution of the equivalent downlink problem. Since both the uplink Problem $P_{1}$ and the equivalent downlink problem achieve the same SINRs and the solution of the downlink problem is optimal, it is straightforward to conclude that Algorithm 1 yields the optimal solution for the considered uplink max-min SINR problem in $P_{1}$. The details of the proof are provided in the following subsections.

\section{A. Equivalent Max-Min Uplink Problem}

In this subsection, we show that both Problem $P_{1}$ with peruser power constraint and the uplink max-min fairness problem with the total power constraint achieve the same user rate. In the total power constraint, the maximum available transmit power is defined as the summation of all users' transmit power from the solution of Problem $P_{1}$, which can be written as follows:

$$
P_{\mathrm{tot}}^{c}=\sum_{k=1}^{K} q_{k}^{*},
$$

where $q_{k}^{*}$ is the power allocated to the $k$ th user obtained by solving problem $P_{1}$ (Algorithm 1). The equivalent uplink maxmin problem with this total power constraint can be formulated as follows:

$$
\begin{gathered}
P_{5}: \max _{q_{k}, \mathbf{u}_{k}} \min _{k=1, \cdots, K} R_{k}^{\mathrm{UP}}, \\
\text { s.t. }\left\|\mathbf{u}_{k}\right\|=1, \quad \forall k, \\
\sum_{k=1}^{K} q_{k} \leq P_{\text {tot }}^{c} .
\end{gathered}
$$

Similar to the original Problem $P_{1}$, Problem $P_{5}$ is not jointly convex in terms of receiver filter coefficients $\mathbf{u}_{k}$ and power allocation $q_{k}, \forall k$. However, we modify Algorithm 1 to incorporate the total power constraint in Problem $P_{5}$. Similar to the 
alternate optimization approach for Problem $P_{1}$, Problem $P_{5}$ is decoupled into receiver filter coefficient design and power allocation sub problems. The same generalized eigenvalue problem in Problem $P_{2}$ is solved to determine the receiver filter coefficients whereas the GP formulation in $P_{4}$ is adapted to incorporate the total power constraint (17c). This is a convex constraint (posynomial function in terms of power allocation) and the power allocation problem (GP) with the equivalent total power constraint remains as a convex problem.

Lemma 1: Both the original Problem $P_{1}$ and Problem $P_{5}$ yield the same solution with per-user power constraint and equivalent total power constraint.

Proof: Please refer to Appendix C.

\section{B. Uplink-Downlink Duality for Cell-Free Massive MIMO}

In this subsection, we establish an uplink-downlink duality for cell-free Massive MIMO systems. In particular, it is shown that the same SINRs (or rate regions) can be realized for all users in the uplink and the downlink with the equivalent total power constraints, respectively [22]-[24], [32]. In other words, the same set of filter coefficients can be utilized in the uplink and the downlink to achieve the same SINRs for all users with different user power allocations. The following theorem defines the achievable downlink rate for cell-free Massive MIMO systems:

Theorem 2: By employing conjugate beamforming at the APs, the achievable downlink rate of the kth user in the cell-free Massive MIMO system with $K$ randomly distributed single-antenna users and $M$ single-antenna APs is given by (18), shown at the bottom of this page.

Proof: This can be derived by following the same approach as for the uplink in Theorem 1.

Note that the symbol $\boldsymbol{\Lambda}_{k^{\prime} k}$, in (18), is defined as $\boldsymbol{\Lambda}_{k^{\prime} k}=\left[\frac{\gamma_{1 k^{\prime}} \beta_{1 k}}{\beta_{1 k^{\prime}}}, \frac{\gamma_{2 k^{\prime}} \beta_{2 k}}{\beta_{2 k^{\prime}}}, \cdots, \frac{\gamma_{M k^{\prime}} \beta_{M k}}{\beta_{M k^{\prime}}}\right]^{T}$, and $\boldsymbol{\Upsilon}_{k^{\prime} k}$ denotes the diagonal matrix whose diagonal entries are $\left[\gamma_{1 k^{\prime}} \beta_{1 k}, \gamma_{2 k^{\prime}} \beta_{2 k}, \cdots, \gamma_{M k^{\prime}} \beta_{M k}\right]$. In addition, $p_{k}, \forall k$ denotes the downlink power allocation for the $k$ th user. Moreover, the uplink SINR is given in (19), shown at the bottom of this page. The following Theorem provides the required condition to establish the uplink-downlink duality for cell-free Massive MIMO systems:

Theorem 3: By employing matched filtering in the uplink and conjugate beamforming in the downlink, to realize the same SINR tuples in both the uplink and the downlink of a cell-free Massive MIMO system, with the same filter coefficients and different transmit power allocations, the following condition should be satisfied:

$$
\sum_{m=1}^{M} \sum_{k=1}^{K} \gamma_{m k}\left|w_{m k}\right|^{2}=\sum_{k=1}^{K} q_{k}^{*}=P_{t o t}^{c},
$$

where $w_{m k}$ denotes the $(m, k)$-th entry of matrix $\boldsymbol{W}$ which is defined as follows:

$$
\boldsymbol{W}=\left[\sqrt{p_{1}} \boldsymbol{u}_{1}, \sqrt{p_{2}} \boldsymbol{u}_{2}, \cdots, \sqrt{p_{K}} \boldsymbol{u}_{K}\right] .
$$

Proof: Please refer to Appendix D.

\section{Equivalent Max-Min Downlink Problem}

In this subsection, we present an optimal approach to solve the max-min SINR downlink problem with the equivalent total power constraint. This user-fairness problem can be formulated as follows:

$$
\begin{gathered}
P_{6}: \max _{p_{k}, \mathbf{u}_{k}} \min _{k=1, \cdots, K} R_{k}^{\mathrm{DL}}, \\
\text { s.t. }\left\|\mathbf{u}_{k}\right\|=1, \quad \forall k, \\
\sum_{k=1}^{K} p_{k} \leq P_{\text {tot }}^{c},
\end{gathered}
$$

where $R_{k}^{\mathrm{DL}}=\log _{2}\left(1+\operatorname{SINR}_{k}^{\mathrm{DL}}\right)$, and $\operatorname{SINR}_{k}^{\mathrm{DL}}$ is defined in (18). This problem is difficult to jointly solve in terms of transmit filter coefficients $\mathbf{u}_{k}$ 's and power allocations $p_{k}$ 's. However, similar to [4], it can be reformulated by introducing a new variable by coupling both of these variables as follows:

$$
\begin{aligned}
P_{7}: & \max _{\mathbf{W}} \min _{k=1, \cdots, K} R_{k}^{\mathrm{DL}}, \\
& \text { s.t. } \sum_{m=1}^{M} \sum_{k=1}^{K} \gamma_{m k}\left|w_{m k}\right|^{2} \leq P_{\text {tot }}^{c} .
\end{aligned}
$$

$$
\begin{array}{ll}
\text { s.t. } & \frac{\mathbf{w}_{k}^{H}\left(\boldsymbol{\Gamma}_{k} \boldsymbol{\Gamma}_{k}^{H}\right) \mathbf{w}_{k}}{\sum_{k^{\prime} \neq k}^{K} \mathbf{w}_{k^{\prime}}^{H}\left|\boldsymbol{\phi}_{k^{\prime}}^{H} \boldsymbol{\phi}_{k}\right|^{2} \boldsymbol{\Lambda}_{k^{\prime} k} \boldsymbol{\Lambda}_{k^{\prime} k}^{H} \mathbf{w}_{k^{\prime}}+\sum_{k^{\prime}=1}^{K} \mathbf{w}_{k^{\prime}}^{H} \mathbf{\Upsilon}_{k^{\prime} k} \mathbf{w}_{k^{\prime}}+\frac{1}{\rho}} \geq t, \\
& \sum_{m=1}^{M} \sum_{k=1}^{K} \gamma_{m k}\left|w_{m k}\right|^{2} \leq P_{\text {tot }}^{c},
\end{array}
$$


It can be easily shown that Problem $P_{7}$ is quasi-convex, therefore a bisection approach can be exploited to obtain the optimal solution for the original Problem $P_{7}$ by sequentially solving the following power minimization problem for a given target SINR $t$ at all users (24a)-(24c), shown at the bottom of this page, where $\mathbf{w}_{k}$ represents the $k$ th column of the matrix $\mathbf{W}$ defined in (21). Second order cone programming (SOCP) can be exploited to reformulate Problem $P_{8}$ as a convex one. More precisely, for a given $t$, Problem $P_{8}$ can be reformulated as follows:

$$
\begin{aligned}
P_{8}^{\text {rewrite }}: \min _{\mathbf{W}} & \sum_{m=1}^{M} \sum_{k=1}^{K} \gamma_{m k}\left|w_{m k}\right|^{2}, \\
\text { s.t. } & \left\|\mathbf{z}_{k}\right\| \leq \frac{\sum_{m=1}^{M}\left[\boldsymbol{\Gamma}_{k}\right]_{m} w_{m k}}{\sqrt{t}}, \forall k, \\
& \sum_{m=1}^{M}\left[\boldsymbol{\Lambda}_{k^{\prime} k}\right]_{m} w_{m k^{\prime}} \leq \chi_{k^{\prime} k}, \forall k^{\prime} \neq k, \\
& \sum_{m=1}^{M}\left[\mathbf{\Upsilon}_{k^{\prime} k}\right]_{m} w_{m k^{\prime}}^{2} \leq \psi_{k^{\prime} k}^{2}, \forall k, \\
& \sum_{m=1}^{M} \sum_{k=1}^{K} \gamma_{m k}\left|w_{m k}\right|^{2} \leq P_{\text {tot }}^{c},
\end{aligned}
$$

where $\chi_{k^{\prime} k}$ and $\psi_{k^{\prime} k}^{2}$ are slack variables, and $[\mathbf{x}]_{n}$ represents the $n$th element of vector $\mathbf{x}$. Moreover, we have

$$
\begin{array}{r}
\mathbf{z}_{k} \triangleq\left[\chi_{1 k} \boldsymbol{\phi}_{1}^{H} \boldsymbol{\phi}_{k}, \cdots, \chi_{(k-1) k} \boldsymbol{\phi}_{k-1}^{H} \boldsymbol{\phi}_{k}, \chi_{(k+1) k} \boldsymbol{\phi}_{k+1}^{H} \boldsymbol{\phi}_{k}, \cdots,\right. \\
\left.\chi_{K k} \boldsymbol{\phi}_{K}^{H} \boldsymbol{\phi}_{k}, \psi_{1 k}, \cdots, \psi_{K k}, \frac{1}{\sqrt{\rho}}\right] . \quad(26)
\end{array}
$$

It can be seen that (25b) represents second order cone (SOC) [33]. Hence, Problem $P_{8}^{\text {rewrite }}$ is a SOCP.

Therefore, the optimal solution for Problem $P_{6}$ can be derived by extracting the normalized transmit filter coefficients $\mathbf{u}_{k}$ 's and power allocations $p_{k}$ 's as

$$
\begin{aligned}
& p_{k}^{*}=\left\|\mathbf{w}_{k}^{*}\right\|^{2}, \quad \forall k, \\
& \mathbf{u}_{k}^{*}=\frac{\mathbf{w}_{k}^{*}}{\left\|\mathbf{w}_{k}^{*}\right\|}, \quad \forall k,
\end{aligned}
$$

where $\mathbf{w}_{k}^{*}$ 's are the optimal solution of Problem $P_{7}$. Note that constraint (23b) is an equivalent total power constraint to the per-user power constraint in the original uplink maxmin SINR problem in $P_{1}$, which is a more relaxed constraint than the per-user power constraint in $P_{1}$. However, it is already shown in the previous sub-section that the same SINRs can be realized in both the uplink and the downlink with peruser and the equivalent total power constraints. In addition, the SINRs achieved in the downlink problem in $P_{7}$ are optimal and therefore the SINRs achieved in Problem $P_{1}$ is optimal. Next, let us again consider the uplink max-min SINR Problems $P_{1}$ and $P_{5}$. After solving the uplink maxmin SINR with total power (with the maximum available power $P_{\text {tot }}^{c}=\sum_{k=1}^{K} q_{k}^{*}$ defined in Problem $P_{5}$ ), and solving the uplink max-min SINR with per-user power constraints (Problem $P_{1}$ ), we observe that the obtained power allocation for all users $\left(q_{k}, \forall k\right)$ after solving Problem $P_{1}$ and Problem $P_{5}$ are exactly the same. Moreover, after solving Problem $P_{5}$ using the proposed Algorithm 1, it is observed that at least one of the users always consumes the maximum power (i.e., there always exists one user with $\left.q_{k}^{*}=p_{\max }^{(k)}\right)$. However, it is easy to prove that it is not possible to improve the max-min rate of the system by increasing the power of other users since in this case we would have to decrease the power of user with $q_{k}^{*}=p_{\max }^{(k)}$, which decreases the rate of this user, and hence the max-min rate. This validates the optimality of the proposed max-min SINR scheme in Algorithm 1.

\section{Sub-Optimal UPLINK MAX-MIN SINR}

In this section, we revisit the bisection search based uplink max-min SINR scheme presented in [4]. First, this bisection scheme is summarized and then, we propose another approach to solve this max-min SINR problem by formulating it into a convex optimization framework. This scheme is developed by appropriately allocating transmit powers at each user with an matched filtering technique at the APs. However, no receiver filter coefficient design has been considered at the CPU to enhance the uplink rate as in the previous section. The achievable rate of the $k$ th user is derived in (28), shown at the bottom of this page, where $\eta_{k}$ is the allocated transmit power at user $k$ [4]. For this scenario, the uplink max-min SINR problem can be formulated as the following max-min problem:

$$
\begin{aligned}
P_{9}: & \max _{\eta_{k} \geq 0} \min _{k} R_{k}^{\mathrm{UP}}, \\
& \text { s.t. } 0 \leq \eta_{k} \leq p_{\max }^{(k)} .
\end{aligned}
$$

\section{A. Bisection Search Method}

In this subsection, we present the bisection search method for this quasi-linear problem. As this problem cannot be directly solved in this present form, a series of power minimization problems is solved by setting the same target rate for all users and the corresponding target rate is modified in the next iteration according to the feasibility or infeasibility of the power minimization problem at each iteration. The feasibility of the following power minimization problem is verified for a given target SINR $t$ at all users in each iteration of the bisection search [4]:

$$
\begin{aligned}
P_{10}: & \min _{\eta_{k}} \sum_{k=1}^{K} \eta_{k}, \\
\text { s.t. } & 0 \leq \eta_{k} \leq p_{\max }^{(k)}, \quad \forall k,
\end{aligned}
$$

$$
\rho \sum_{k^{\prime} \neq k}^{K} \eta_{k^{\prime}}\left(\sum_{m=1}^{M} \gamma_{m k} \frac{\beta_{m k^{\prime}}}{\beta_{m k}}\right)^{2}\left|\boldsymbol{\phi}_{k}^{H} \boldsymbol{\phi}_{k^{\prime}}\right|^{2} t
$$

$$
R_{k}^{\mathrm{UP}}=\left(1+\frac{\rho \eta_{k}\left(\sum_{m=1}^{M} \gamma_{m k}\right)^{2}}{\rho \sum_{k^{\prime} \neq k}^{K} \eta_{k^{\prime}}\left(\sum_{m=1}^{M} \gamma_{m k} \frac{\beta_{m k^{\prime}}}{\beta_{m k}}\right)^{2}\left|\boldsymbol{\phi}_{k}^{H} \boldsymbol{\phi}_{k^{\prime}}\right|^{2}+\rho \sum_{k^{\prime}=1}^{K} \eta_{k^{\prime}} \sum_{m=1}^{M} \gamma_{m k} \beta_{m k^{\prime}}+\sum_{m=1}^{M} \gamma_{m k}}\right) .
$$




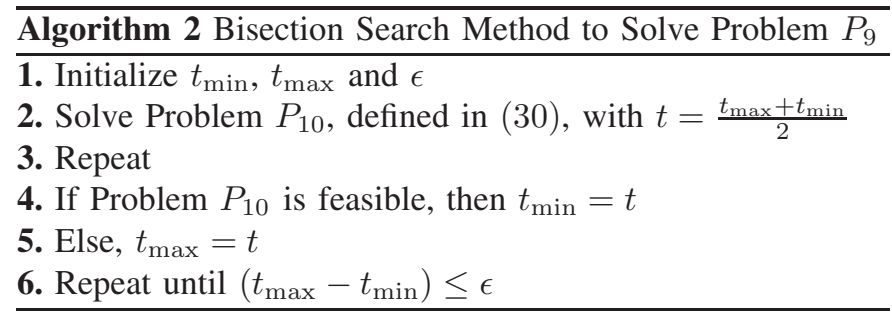

$$
\begin{aligned}
& +\rho \sum_{k^{\prime}=1}^{K} \eta_{k^{\prime}} \sum_{m=1}^{M} \gamma_{m k} \beta_{m k^{\prime}} t+\sum_{m=1}^{M} \gamma_{m k} t \\
& \leq \rho \eta_{k}\left(\sum_{m=1}^{M} \gamma_{m k}\right)^{2}, \forall k .
\end{aligned}
$$

In this bisection search approach, first an upper and lower bounds of the achievable SINR are set to $t_{\max }$ and $t_{\min }$, respectively and the initial target SINR $t$ is chosen as $\left(t_{\max }+\right.$ $\left.t_{\text {min }}\right) / 2$. If Problem $P_{10}$ is feasible for a given target SINR $t$, then the lower bound $t_{\min }$ will be set to $t$ and a new target SINR is chosen as $\left(t_{\max }+t_{\min }\right) / 2$ for the next iteration. This procedure is continued until the difference between the upper and the lower bounds is smaller than a predefined threshold $\epsilon$. This bisection search method based uplink max-min SINR scheme is summarized in Algorithm 2. Note that based on the analysis in [17], the bisection search method provides the optimal solution. In the rest of this section, we show that Problem $P_{9}$ can be reformulated as a standard GP, which does not require an iterative bisection search to find the optimal solution.

\section{B. Proposed Sub-Optimal Scheme}

In this subsection, we exploit GP (convex problem) to develop an efficient solution for Problem $P_{9}$ defined in (29). As mentioned in previous subsection, Problem $P_{9}$ cannot be directly solved through the optimization software. Consider the following optimization problem:

$$
\begin{aligned}
P_{11}: & \max _{t, \eta_{k}} t, \\
& \text { s.t. } 0 \leq \eta_{k} \leq p_{\max }^{(k)}, \quad \forall k, \\
& \operatorname{SINR}_{k}^{\mathrm{UP}} \geq t, \quad \forall k .
\end{aligned}
$$

Proposition 2: Problem $P_{11}$ can be reformulated into a GP.

Proof: The standard form of GP is defined in Appendix B. The SINR constraint in (31c) can be reformulated into the posynomial function. Following a simple transformation, the SINR constraint in (31c) can be represented by the following inequality:

$$
\eta_{k}^{-1}\left(\sum_{k^{\prime} \neq k}^{K} e_{k k^{\prime}} \eta_{k^{\prime}}+\sum_{k^{\prime}=1}^{K} f_{k k^{\prime}} \eta_{k^{\prime}}+r_{k}\right)<\frac{1}{t},
$$

where

$$
e_{k k^{\prime}}=\frac{\left(\sum_{m=1}^{M} \gamma_{m k} \frac{\beta_{m k^{\prime}}}{\beta_{m k}}\right)^{2}\left|\phi_{k}^{H} \phi_{k^{\prime}}\right|^{2}}{\left(\sum_{m=1}^{M} \gamma_{m k}\right)^{2}}
$$

TABLE I

Computational Complexity of Different Problems

\begin{tabular}{lc}
\hline Problems & Required arithmetic operations \\
\hline Problem $P_{2}$, given by (13) & $O\left(K M^{3}\right)$ \\
\hline Problem $P_{4}$, given by (15) & $O\left(K^{\frac{7}{2}}\right)$ \\
\hline Problem $P_{10}$, given by (30) & $\log _{2}\left(\frac{t_{\max }-t_{\min }}{\epsilon}\right) O\left(K^{4}\right)$ \\
\hline Problem $P_{11}$, given by (31) & $O\left(K^{\frac{7}{2}}\right)$ \\
\hline \multicolumn{3}{c}{$f_{k k^{\prime}}=\frac{\sum_{m=1}^{M} \gamma_{m k} \beta_{m k^{\prime}}}{\left(\sum_{m=1}^{M} \gamma_{m k}\right)^{2}}}$, \\
$r_{k}=\frac{(33 \mathrm{~b})}{\rho\left(\sum_{m=1}^{M} \gamma_{m k}\right.}$ \\
\end{tabular}

The transformation in (32) demonstrates that the left-hand side of (32) is a posynomial function. Hence, Problem $P_{11}$ is a standard GP, which completes the proof of Proposition 2.

Based on Proposition 2, the objective function and constraints of Problem $P_{11}$ are monomial and posynomials functions in terms of power allocaitons $\eta_{k}$ s. Hence, Problem $P_{11}$ is a standard GP, and can be efficiently solved through convex optimization software. Simulation results are provided to show that both bisection and GP based sub-optimal schemes achieve the same user rate for all users.

\section{COMPLEXITy ANALYSIS}

Here, we provide the computational complexity analysis for the proposed Algorithm 1, which solves a generalized eigenvalue problem $P_{2}$ and a GP (convex optimization problem) $P_{4}$ at each iteration. For the receiver filter coefficient design in $P_{2}$, given by (13), an eigenvalue solver requires approximately $\mathcal{O}\left(K M^{3}\right)$ flops [34], [35]. Note that the complexity analysis of an eigenvalue solver takes into account the matrix inversion as well. In addition, a standard GP in Problem $P_{4}$, defined in (15), can be solved with complexity equivalent to $\mathcal{O}\left(K^{\frac{7}{2}}\right)$ [36, Ch. 10]. The proposed sub-optimal scheme in Section VII solves a GP in Problem $P_{11}$, defined in (31), which can be solved with $\mathcal{O}\left(K^{\frac{7}{2}}\right)$ [36, Ch. 10]. However, for the scheme in [4], the iterative bisection search method in Algorithm 2 solves a SOCP at each iteration. The complexity of SOCP is $\mathcal{O}\left(K^{4}\right)$ in each iteration [37], [38]. Note that the total number of iterations to solve Problem $P_{9}$ via a bisection search method is given by $\log _{2}\left(\frac{t_{\max }-t_{\min }}{\epsilon}\right)$, where $\epsilon$ refers to a predetermined threshold [33]. The number of arithmetic operations required for Algorithm 1, Algorithm 2, and the proposed sub-optimal scheme are provided in Table I.

\section{Proposed User Assignment Scheme}

In practice, the total backhaul capacity required between the $m$ th AP and the CPU increases linearly with the total number of users served by the $m$ th AP, which motivates the need to pick a proper set of active users for each AP [28]. In [28], we proposed a user assignment algorithm which can reduce 
the required capacity of backhaul link by assigning a limited number of users to each AP, however, this paper assumes perfect backhaul links. Hence, for simplicity we assume here that only $\operatorname{th}_{m} \%$ of the total number of users can be supported by the $m$ th AP. Hence, we have

$$
K_{m} \leq\left(\frac{\mathrm{th}_{m}}{100} \times K\right),
$$

where $K_{m}$ denotes the size of the set of active users for the $m$ th AP. First, we find an upper bound on the size of the set of active users for each AP. In the next step, we propose for all APs that the users are sorted according to $\beta_{m k}, \forall k$, and find the $K_{m}$ users which have the highest values of $\beta_{m k}$ among all users. If a user is not selected by any AP, we propose to find the AP which has the best link to this user. Then, we add the user to the set of active users for this AP and drop the user which has the lowest $\beta_{m k}, \forall k$, among active users for that AP which have links to other APs as well. We next solve the original max-min SINR problem with $\tilde{\gamma}_{m k} \leftarrow \gamma_{m k}$, where $\tilde{\gamma}_{m k}$ is given by

$$
\tilde{\gamma}_{m k}= \begin{cases}\gamma_{m k}, & m \in \mathcal{S}_{k} \\ 0, & \text { otherwise }\end{cases}
$$

where $\mathcal{S}_{k}$ refers to the set of active APs for the $k$ th user. Note that optimum user assignment scheme can be considered in future work.

\section{Numerical Results AND Discussion}

In this section, we provide numerical simulation results to validate the performance of the proposed max-min SINR scheme with different parameters. A cell-free Massive MIMO system with $M$ APs and $K$ single-antenna users is considered in a $D \times D$ simulation area, where both APs and users are uniformly located at random. In the following subsections, we define the simulation parameters and then present the corresponding simulation results.

\section{A. Simulation Parameters}

The channel coefficients between users and APs are modeled in (1) where the coefficient $\beta_{m k}$ is given by [4]

$$
\beta_{m k}=\mathrm{PL}_{m k} 10 \frac{\sigma_{s h} z_{m k}}{10},
$$

where $\mathrm{PL}_{m k}$ is the path loss from the $k$ th user to the $m$ th $\mathrm{AP}$ and the second term in (36), $10^{\frac{\sigma_{s h} z_{m k}}{10}}$, denotes the shadow fading with standard deviation $\sigma_{s h}=8 \mathrm{~dB}$, and $z_{m k} \sim$ $\mathcal{N}(0,1)$. In the simulation, an uncorrelated shadowing model is considered and a three-slope model for the path loss is given by [4], [39]

$$
\mathrm{PL}_{m k}= \begin{cases}-L-35 \log _{10}\left(d_{m k}\right), & d_{m k}>d_{1}, \\ -L-15 \log _{10}\left(d_{1}\right)-20 \log _{10}\left(d_{m k}\right), & d_{0}<d_{m k} \leq d_{1}, \\ -L-15 \log _{10}\left(d_{1}\right)-20 \log _{10}\left(d_{0}\right), & d_{m k} \leq d_{0},\end{cases}
$$

and $L=46.3+33.9 \log _{10}(f)-13.82 \log _{10}\left(h_{A P}\right)-$ $\left(1.1 \log _{10}(f)-0.7\right) h_{k}+\left(1.56 \log _{10}(f)-0.8\right)$, where $f$ denotes the carrier frequency (in MHz), $h_{A P}$ and $h_{k}$ represent the AP antenna height (in $\mathrm{m}$ ) and user height (in $\mathrm{m}$ ),

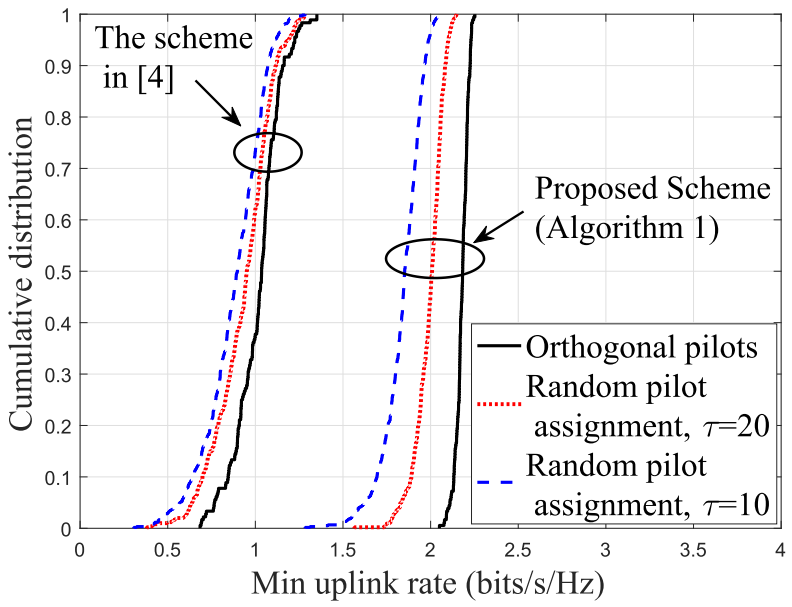

Fig. 2. The cumulative distribution of the min uplink rate, with orthogonal and random pilots for $M=120, K=30$ and $D=1 \mathrm{~km}^{2}$.

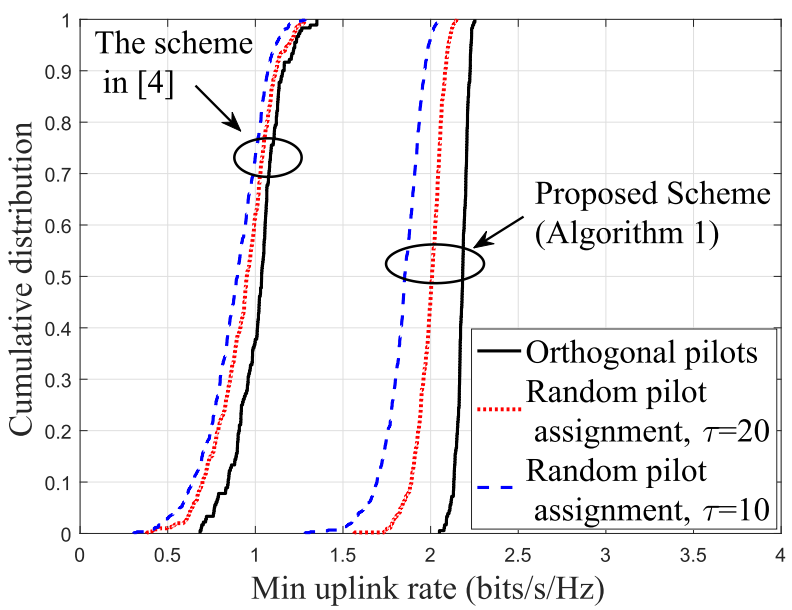

Fig. 3. The cumulative distribution of the min uplink rate, with orthogonal and random pilots for $M=120, K=30$ and $D=1 \mathrm{~km}^{2}$.

respectively. The noise power is given by $p_{n}=\mathrm{BW} \times k_{B} \times$ $T_{0} \times W$, where $\mathrm{BW}=20 \mathrm{MHz}$ denotes the bandwidth, $k_{B}=1.381 \times 10^{-23}$ represents the Boltzmann constant, and $T_{0}=290$ (Kelvin) denotes the noise temperature. Moreover, $W=9 \mathrm{~dB}$, and denotes the noise figure. It is assumed that that $\bar{p}_{p}$ and $\bar{\rho}$ denote the pilot sequence and the uplink data powers, respectively, where $p_{p}=\frac{\bar{p}_{p}}{p_{n}}$ and $\rho=\frac{\bar{\rho}}{p_{n}}$. In simulations, we set $\bar{p}_{p}=200 \mathrm{~mW}$ and $\bar{\rho}=200 \mathrm{~mW}$. Similar to [4], we assume that the simulation area is wrapped around at the edges which can simulate an area without boundaries. Hence, the square simulation area has eight neighbours. We evaluate the average rate of the system over 300 random realizations of the locations of APs, users and shadow fading. Furthermore, to consider the channel estimation overhead in our comparison, we exploit the net throughput of the system which is defined as [4] $R_{\text {net, } \mathrm{k}}=\mathrm{BW} \frac{1-\frac{\tau}{\tau_{c}}}{2} R_{k}$, where $\tau_{c}$ represents the coherence interval in samples.

\section{B. Simulation Results}

1) Performance of the Proposed Max-Min SINR Algorithm: In this subsection, we evaluate the performance of the proposed uplink max-min SINR scheme. To assess the perfor- 


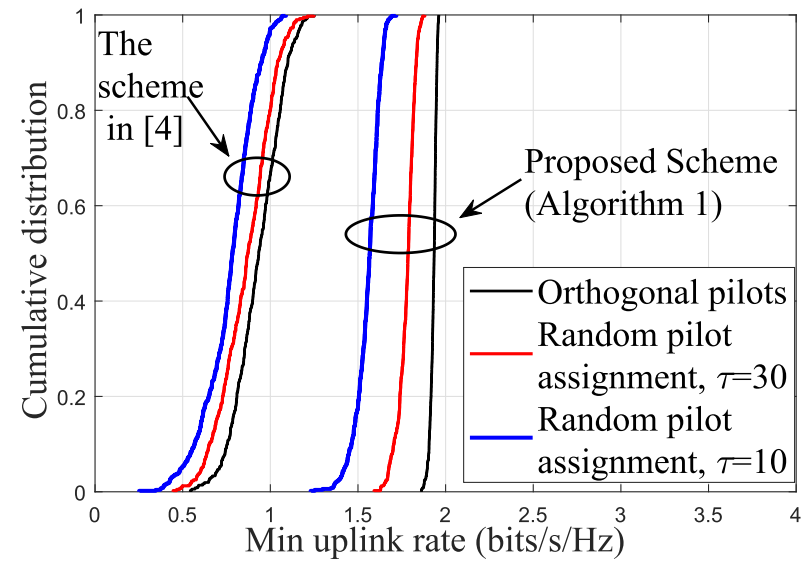

Fig. 4. The cumulative distribution of the min uplink rate, with random pilots for $M=150, K=50$ and $D=1 \mathrm{~km}^{2}$.

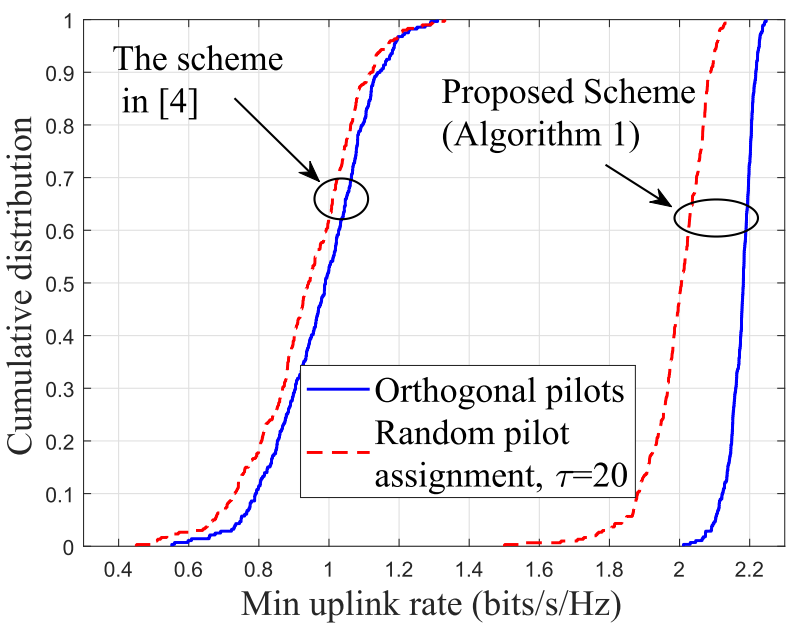

Fig. 5. The cumulative distribution of the min uplink rate with proposed user assignment scheme in Section IX, with orthogonal and random pilots for $M=120, K=30, K_{m}=20, \forall m$, and $D=1 \mathrm{~km}^{2}$. mance, a cell-free Massive MIMO system is considered with 120 APs $(M=120)$ and 30 users $(K=30)$ who are randomly distributed over the simulation area of size $1 \times 1 \mathrm{~km}^{2}$. Fig. 3 presents the cumulative distribution of the achievable uplink rates for the proposed Algorithm 1 and the scheme in [4], for the cases of orthogonal and random pilots. As seen in Fig. 3, the performance of the proposed scheme is almost three times than that of the scheme in [4]. Next, the performance of the algorithm is evaluated for a system with 150 APs $(M=150)$ and 50 users $(K=50) .{ }^{1}$ Fig. 4 similarly compares the rate of the proposed algorithm with the scheme in [4]. The simulation results in Figs. 3 and 4 show that the proposed Algorithm 1 achieves more than double the $10 \%$ outage capacity compared to the scheme in [4]. Moreover, Figs. 3 and 4 demonstrate that the rate of the proposed max-min SINR approach is more concentrated around the median value.

2) User Assignment: In this subsection, the performance of the proposed uplink max-min SINR scheme with the proposed user assignment scheme in Section IX is investigated. We set

${ }^{1}$ The analysis in [40] demonstrates that in the limit of Massive MIMO $\left(M, K \rightarrow \infty\right.$ and $\alpha=\frac{M}{K}$ ), when $\alpha \geq 4$, linear precoding is "virtually optimal", and can be used instead of dirty paper coding (DPC). In this paper, we consider the two cases $\alpha=\frac{120}{30}=4$ and $\alpha=\frac{150}{50}=3$.

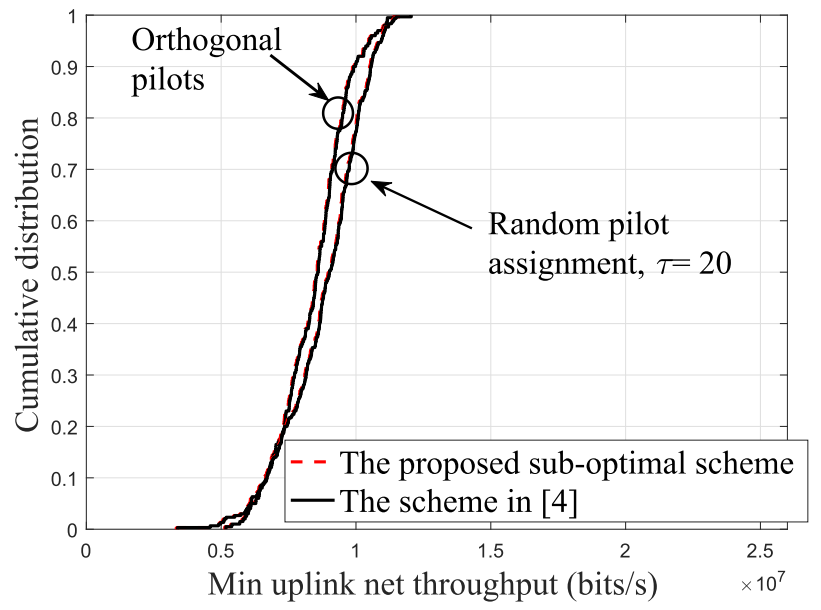

Fig. 6. The cumulative distribution of the min uplink net throughput, with orthogonal and random pilots for $M=120, K=30, D=1 \mathrm{~km}^{2}$ and $\tau_{c}=200$.

120 APs $(M=120)$ and 30 users $(K=30)$, and assume $66.66 \%$ of the total number of users can be supported by each AP. Based on the analysis in Section IX, this results in a total number of users supported users by each AP of $K_{m}=20, \forall m$. Fig. 5 presents the cumulative distribution of the achievable uplink rates for the proposed Algorithm 1 and the scheme in [4] with the proposed user assignment algorithm in Section IX, for the cases of orthogonal and random pilots. As seen in Fig. 5, the performance of the proposed scheme is significantly better than that of the scheme in [4]. In addition, it can be observed from figure that the rate of the proposed Algorithm 1 is more concentrated around the median. Interestingly, by comparing the results in Figs. 3 and 5, the performance degradation is negligible exploiting the proposed user assignment scheme whereas based on the analysis in [28], the backhaul rate is significantly reduced.

3) Performance of the Proposed Sub-Optimal Scheme: In this subsection, we study the effect of the proposed suboptimal scheme on the system performance. Fig. 6 compares the cumulative distribution of the achievable uplink net throughput for our proposed sub-optimal scheme with scheme in [4]. In order to generate the numerical results for the scheme in [4], the iterative bisection search method in Algorithm 2 is used whereas the proposed sub-optimal scheme solves the standard GP with polynomial time complexity. In Fig. 6, the same cell-free Massive MIMO system is considered with 120 APs $(M=120)$ and 30 users $(K=30)$. Figs. 6 and 7 compare the performance of the proposed sub-optimal approach with the scheme in [4] for different system parameters. As evidenced from these numerical results, both proposed GP approach and the bisection search scheme in [4] shows the same performance in terms of the achieved user rate. However, the scheme in [4] is developed through iterative bisection search in which a SOCP is solved at each iteration, whereas the proposed GP approach does not require any iterative methods and solves the problem with polynomial time complexity.

4) Convergence: Next, we provide simulation results to validate the convergence of the proposed algorithm for a set 


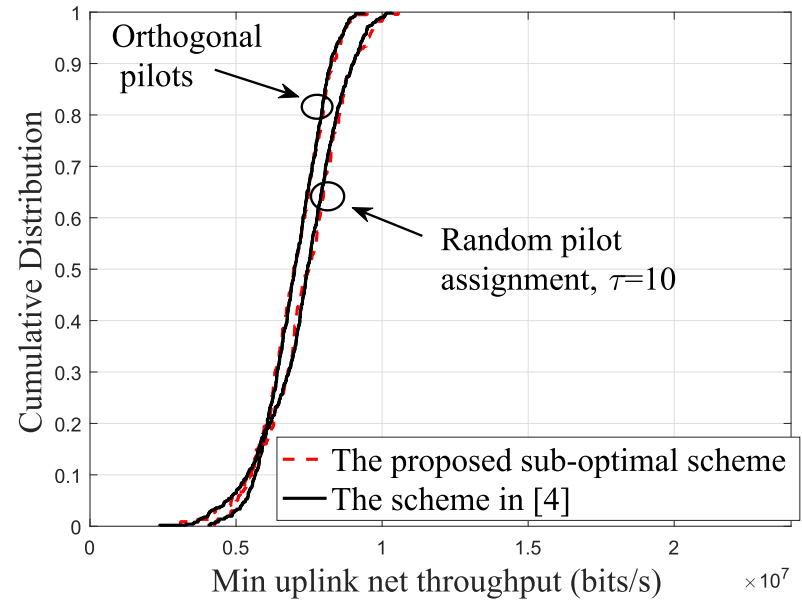

Fig. 7. The cumulative distribution of the min uplink net throughput, with orthogonal and random pilots for $M=150, K=50, D=1 \mathrm{~km}^{2}$ and $\tau_{c}=200$.

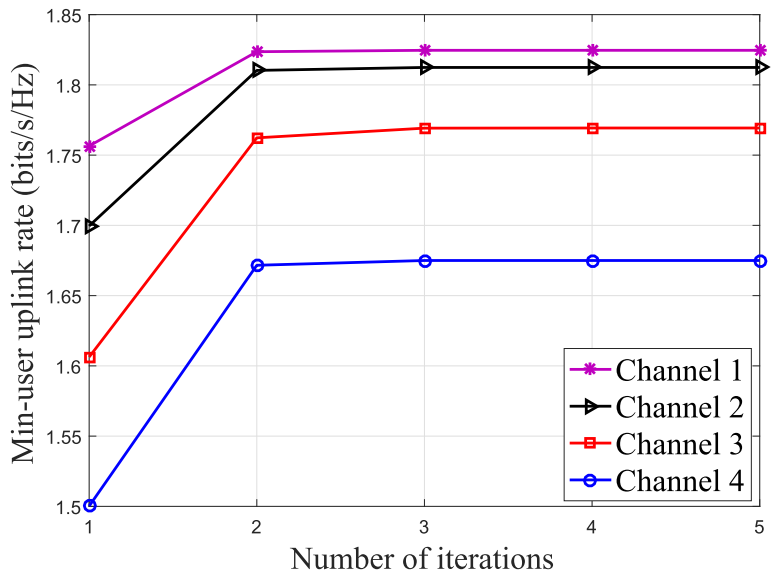

Fig. 8. The convergence of the proposed max-min SINR approach (Algorithm 1) for $M=120, K=30$ and $D=1 \mathrm{~km}$ with orthogonal pilots.

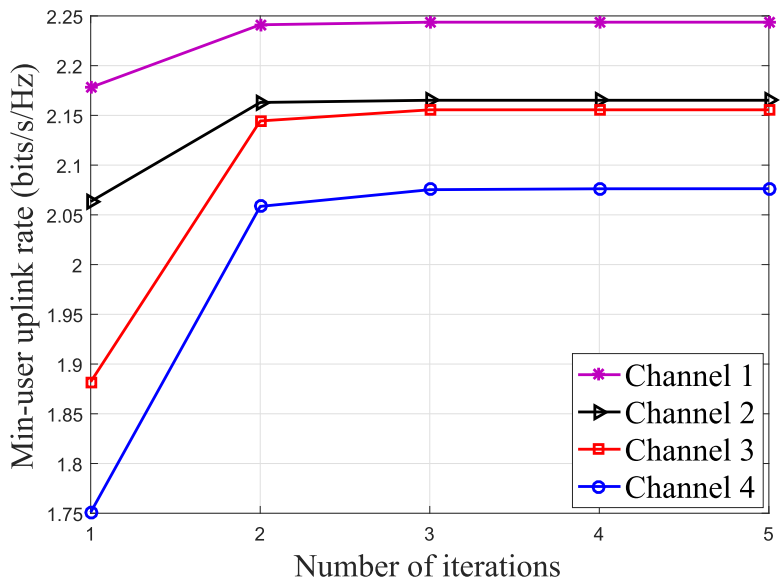

Fig. 9. The convergence of the proposed max-min SINR approach (Algorithm 1) for $M=150, K=50, D=1 \mathrm{~km}$, and the length of the pilot sequences is set to $30(\tau=30)$.

of different channel realizations. These results are generated over the simulation area of size $1 \times 1 \mathrm{~km}^{2}$ with random and orthogonal pilot sequences. Fig. 8 investigates the convergence of the proposed Algorithm 1 with 120 APs $(M=120)$

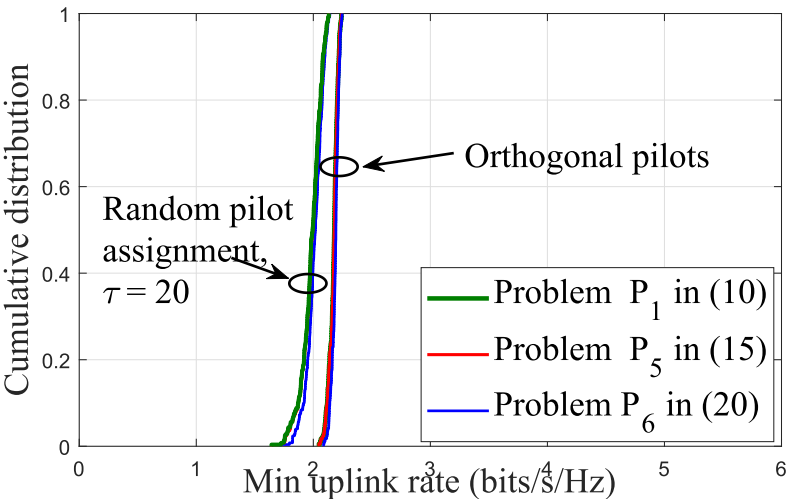

Fig. 10. The cumulative distribution of the min uplink rate for the original problem with per-user power constraint (Problem $P_{1}$ ), the equivalent uplink problem with total power constraint (Problem $P_{5}$ ), and the equivalent downlink problem (Problem $P_{6}$ ), with orthogonal and random pilots for $M=120, K=30$ and $D=1 \mathrm{~km}$.

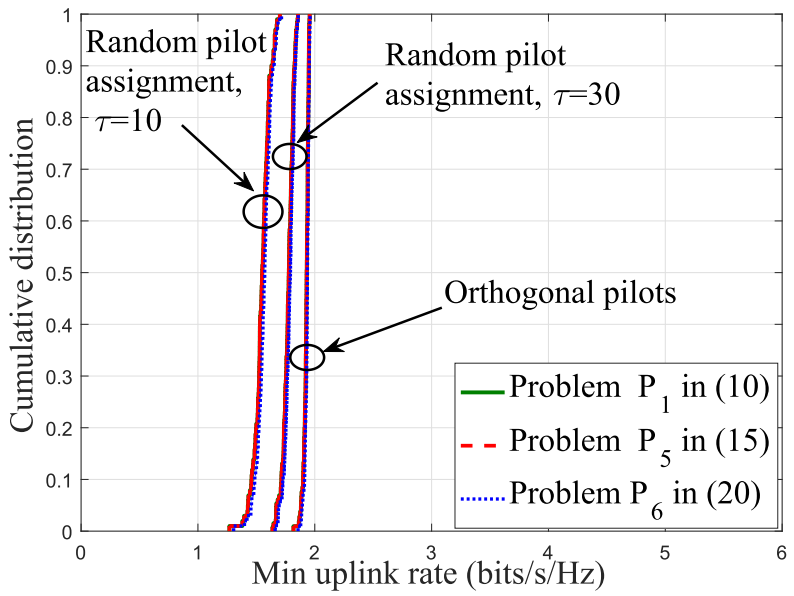

Fig. 11. The cumulative distribution of the min uplink rate for the original problem with per-user power constraint (Problem $P_{1}$ ), the equivalent uplink problem with total power constraint (Problem $P_{5}$ ), and the equivalent downlink problem (Problem $P_{6}$ ), with orthogonal and random pilots for $M=150, K=50$ and $D=1 \mathrm{~km}$.

and 30 users $(K=30)$ and orthogonal pilot sequences, whereas Fig. 9 demonstrates the convergence of the proposed Algorithm 1 for the case of $M=150$ APs and $K=50$. The figures confirm that the proposed algorithm converges after a few iterations, while the minimum rate of the users increases with the iteration number.

5) Uplink-Downlink Duality in Cell-Free Massive MIMO System: Here, the simulation results are provided to support the theoretical derivations of the uplink-downlink duality and the optimality of Algorithm 1. It is assumed that users are randomly distributed through the simulation area of size $1 \times 1 \mathrm{~km}^{2}$. Figs. 10 and 11 compare the cumulative distribution of the achievable uplink rates between the original uplink max-min problem (Problem $P_{1}$ ), the equivalent uplink problem (Problem $P_{5}$ ) and the equivalent downlink problem (Problem $P_{6}$ ). In Fig. 10, the minimum uplink rate is obtained for a system with 120 APs $(M=120)$ and 30 users $(K=30)$ whereas Fig. 11 presents the same results for 150 APs $(M=150)$ and 50 users $(K=50)$. The simulation results provided in Figs. 10 and 11 validate our result that 
the problem formulations $P_{1}, P_{5}$ and $P_{6}$ are equivalent and achieve the same minimum user rate. In addition, these results support our result on the uplink-downlink duality for cell-free Massive MIMO in Section VI and the proof of optimality of Algorithm 1.

\section{Conclusions}

We have considered cell-free Massive MIMO which has the potential to meet the capacity requirements of 5G. Compared to the collocated Massive MIMO, the distributed version brings the APs much closer to the "cell edge" users, which leads to a uniformly good service for all users. We have investigated the uplink max-min SINR problem in cell-free Massive MIMO systems and proposed an optimal solution to maximize the minimum uplink user rate. To realize the solution, the original max-min problem was divided into two sub-problems which were iteratively solved by formulating them respectively as a generalized eigenvalue problem and as GP. The optimality of the proposed solution has been validated by establishing the uplink-downlink duality for cellfree Massive MIMO systems. Next, a novel sub-optimal scheme was developed through formulating the max-min power allocation problem as a standard GP, which efficiently and globally solves the max-min SINR problem. Simulation results have been provided to demonstrate the effectiveness and the optimality of the proposed schemes in comparison with the existing schemes. In addition, these results confirm that the proposed max-min SINR algorithm can significantly improve the uplink user rate, compared to existing algorithms.

\section{APPENDIX A}

\section{PROOF OF THEOREM 1}

The desired signal for user $k$ is given by

$\mathrm{DS}_{k}=\sqrt{\rho} \mathbb{E}\left\{\sum_{m=1}^{M} u_{m k} \hat{g}_{m k}^{*} g_{m k} \sqrt{q_{k}}\right\}=\sqrt{\rho q_{k}} \sum_{m=1}^{M} u_{m k} \gamma_{m k}$.

Hence,

$$
\left|\mathrm{DS}_{k}\right|^{2}=\rho q_{k}\left(\sum_{m=1}^{M} u_{m k} \gamma_{m k}\right)^{2} .
$$

Moreover, the term $\mathbb{E}\left\{\left|\mathrm{BU}_{k}\right|^{2}\right\}$ can be obtained as

$$
\begin{aligned}
\mathbb{E}\left\{\left|\mathrm{BU}_{k}\right|^{2}\right\} & \\
=\rho \mathbb{E} & \left\{\mid \sum_{m=1}^{M} u_{m k} \hat{g}_{m k}^{*} g_{m k} \sqrt{q_{k}}\right. \\
& \left.-\left.\rho \mathbb{E}\left\{\sum_{m=1}^{M} u_{m k} \hat{g}_{m k}^{*} g_{m k} \sqrt{q_{k}}\right\}\right|^{2}\right\} \\
= & \rho \sum_{m=1}^{M} q_{k} u_{m k}^{2}\left(\mathbb{E}\left\{\left|\hat{g}_{m k}^{*} g_{m k}-\mathbb{E}\left\{\hat{g}_{m k}^{*} g_{m k}\right\}\right|^{2}\right\}\right) \\
= & \rho q_{k} \sum_{m=1}^{M} u_{m k}^{2} \gamma_{m k} \beta_{m k},
\end{aligned}
$$

where the last equality comes from the analysis in 835 [4, Appendix A], and using the following fact that; $\gamma_{m k}=$ $\mathbb{E}\left\{\left|\hat{g}_{m k}\right|^{2}\right\}=\sqrt{\tau p_{p}} \beta_{m k} c_{m k}$. The term $\mathbb{E}\left\{\left|\mathrm{IUI}_{k k^{\prime}}\right|^{2}\right\}$ is derived as

$$
\begin{aligned}
\mathbb{E}\left\{\left|\mathrm{IUI}_{k k^{\prime}}\right|^{2}\right\} & \rho \mathbb{E}\left\{\left|\sum_{m=1}^{M} u_{m k} \hat{g}_{m k}^{*} g_{m k^{\prime}} \sqrt{q_{k^{\prime}}}\right|^{2}\right\} \\
= & p \mathbb{E}\left\{\mid \sum_{m=1}^{M} c_{m k} u_{m k} g_{m k^{\prime}} \sqrt{q_{k^{\prime}}}\right. \\
& \left.\times\left.\left(\sqrt{\tau p_{p}} \sum_{i=1}^{K} g_{m i} \boldsymbol{\phi}_{k}^{H} \boldsymbol{\phi}_{i}+\boldsymbol{\phi}_{k}^{H} \mathbf{n}_{p, m}\right)^{*}\right|^{2}\right\} \\
= & \underbrace{\rho q_{k^{\prime}} \mathbb{E}\left\{\left|\sum_{m=1}^{M} c_{m k} u_{m k} g_{m k^{\prime}} \tilde{n}_{m k}^{*}\right|^{2}\right\}}_{B} \underbrace{}_{A} \underbrace{\rho \tau p_{p} \mathbb{E}\left\{q_{k^{\prime}}\left|\sum_{m=1}^{M} c_{m k} u_{m k} g_{m k^{\prime}}\left(\sum_{i=1}^{K} g_{m i} \boldsymbol{\phi}_{k}^{H} \boldsymbol{\phi}_{i}\right)^{*}\right|^{2}\right\}},
\end{aligned}
$$

where the third equality in (41) is due to the fact that for two independent random variables $X$ and $Y$ and $\mathbb{E}\{X\}=$ 0 , we have $\mathbb{E}\left\{|X+Y|^{2}\right\}=\mathbb{E}\left\{|X|^{2}\right\}+\mathbb{E}\left\{|Y|^{2}\right\}$ [4]. Since $\tilde{n}_{m k}=\boldsymbol{\phi}_{k}^{H} \mathbf{n}_{p, m} \sim \mathcal{C N}(0,1)$ is independent of the term $g_{m k^{\prime}}$, the term $A$ in (41) is given immediately by

$$
A=q_{k^{\prime}} \sum_{m=1}^{M} c_{m k}^{2} u_{m k}^{2} \beta_{m k^{\prime}} .
$$

The term $B$ in (41) can be obtained as

$$
\begin{aligned}
B & =\underbrace{\tau p_{p} q_{k^{\prime}} \mathbb{E}\left\{\left.\left.\left|\sum_{m=1}^{M} c_{m k} u_{m k}\right| g_{m k^{\prime}}\right|^{2} \boldsymbol{\phi}_{k}^{H} \boldsymbol{\phi}_{k^{\prime}}\right|^{2}\right\}}_{C} \\
& +\underbrace{\tau p_{p} q_{k^{\prime}} \mathbb{E}\left\{\left|\sum_{m=1}^{M} c_{m k} u_{m k} g_{m k^{\prime}}\left(\sum_{i \neq k^{\prime}}^{K} g_{m i} \boldsymbol{\phi}_{k}^{H} \boldsymbol{\phi}_{i}\right)^{*}\right|^{2}\right\}}_{D} .
\end{aligned}
$$

The first term in (43) is given by

$$
\begin{aligned}
C= & \tau p_{p} q_{k^{\prime}} \mathbb{E}\left\{\left.\left.\left|\sum_{m=1}^{M} c_{m k} u_{m k}\right| g_{m k^{\prime}}\right|^{2} \boldsymbol{\phi}_{k}^{H} \boldsymbol{\phi}_{k^{\prime}}\right|^{2}\right\} \\
= & 2 \tau p_{p} q_{k^{\prime}}\left|\boldsymbol{\phi}_{k}^{H} \boldsymbol{\phi}_{k^{\prime}}\right|^{2} \sum_{m=1}^{M} c_{m k}^{2} u_{m k}^{2} \beta_{m k^{\prime}}^{2}+\tau p_{p} q_{k^{\prime}} \\
& \mathbb{E}\left\{\left|\boldsymbol{\phi}_{k}^{H} \boldsymbol{\phi}_{k^{\prime}}\right|^{2} \sum_{m=1}^{M} \sum_{n \neq m}^{M} c_{m k} c_{n k} u_{m k} u_{n k}\left|g_{m k^{\prime}}\right|^{2}\left|g_{n k^{\prime}}\right|^{2}\right\} \\
= & \tau p_{p} q_{k^{\prime}}\left|\boldsymbol{\phi}_{k}^{H} \boldsymbol{\phi}_{k^{\prime}}\right|^{2} \sum_{m=1}^{M} c_{m k}^{2} u_{m k}^{2} \beta_{m k^{\prime}}^{2} \\
+ & q_{k^{\prime}}\left|\boldsymbol{\phi}_{k}^{H} \boldsymbol{\phi}_{k^{\prime}}\right|^{2}\left(\sum_{m=1}^{M} u_{m k} \gamma_{m k} \frac{\beta_{m k^{\prime}}}{\beta_{m k}}\right)^{2}
\end{aligned}
$$


where the last equality is derived based on the fact $\gamma_{m k}=$ $\sqrt{\tau p_{p}} \beta_{m k} c_{m k}$. The second term in (43) can be obtained as

$$
\begin{aligned}
D & =\tau p_{p} q_{k^{\prime}} \mathbb{E}\left\{\left|\sum_{m=1}^{M} c_{m k} u_{m k} g_{m k^{\prime}}\left(\sum_{i \neq k^{\prime}}^{K} g_{m i} \boldsymbol{\phi}_{k}^{H} \boldsymbol{\phi}_{i}\right)^{*}\right|^{2}\right\} \\
& =\tau p_{p} \sum_{m=1}^{M} \sum_{i \neq k^{\prime}}^{K} q_{k^{\prime}} c_{m k}^{2} u_{m k}^{2} \beta_{m k^{\prime}} \beta_{m i}\left|\boldsymbol{\phi}_{k}^{H} \boldsymbol{\phi}_{i}\right|^{2} .
\end{aligned}
$$

Hence, (41) can be written as

$$
\begin{aligned}
\mathbb{E}\left\{\left|\mathrm{IUI}_{k k^{\prime}}\right|^{2}\right\}= & \underbrace{q_{k^{\prime}} \sum_{m=1}^{M} c_{m k}^{2} u_{m k}^{2} \beta_{m k^{\prime}}}_{C_{1}} \\
& +\tau p_{p} q_{k^{\prime}}\left|\boldsymbol{\phi}_{k}^{H} \boldsymbol{\phi}_{k^{\prime}}\right|^{2} \sum_{m=1}^{M} c_{m k}^{2} u_{m k}^{2} \beta_{m k^{\prime}}^{2} \\
& +\tau p_{p} q_{k^{\prime}} \sum_{m=1}^{M} \sum_{i \neq k^{\prime}}^{K} c_{m k}^{2} u_{m k}^{2} \beta_{m k^{\prime}} \beta_{m i}\left|\boldsymbol{\phi}_{k}^{H} \boldsymbol{\phi}_{i}\right|^{2} \\
& q_{k^{\prime}}\left|\boldsymbol{\phi}_{k}^{H} \boldsymbol{\phi}_{k^{\prime}}\right|^{2}\left(\sum_{m=1}^{M} u_{m k} \gamma_{m k} \frac{\beta_{m k^{\prime}}}{\beta_{m k}}\right)^{2},
\end{aligned}
$$

and

$$
\begin{aligned}
C_{2}= & \tau p_{p} q_{k^{\prime}}\left|\boldsymbol{\phi}_{k}^{H} \boldsymbol{\phi}_{k^{\prime}}\right|^{2} \sum_{m=1}^{M} c_{m k}^{2} u_{m k}^{2} \beta_{m k^{\prime}}^{2} \\
& +\underbrace{\tau p_{p} q_{k^{\prime}} \sum_{m=1}^{M} \sum_{i \neq k^{\prime}}^{M} c_{m k}^{2} u_{m k}^{2} \beta_{m k^{\prime}} \beta_{m i}\left|\boldsymbol{\phi}_{k}^{H} \boldsymbol{\phi}_{i}\right|^{2}}_{C_{3}} .
\end{aligned}
$$

For the last term of (47), we have

$$
\begin{aligned}
& C_{3}=\tau p_{p} q_{k^{\prime}} \sum_{m=1}^{M} \sum_{i \neq k^{\prime}}^{K} c_{m k}^{2} u_{m k}^{2} \beta_{m k^{\prime}} \beta_{m i}\left|\phi_{k}^{H} \boldsymbol{\phi}_{i}\right|^{2} \\
&= \tau p_{p} q_{k^{\prime}}\left(\sum_{m=1}^{M} u_{m k}^{2} c_{m k} \beta_{m k^{\prime}} \sum_{i=1}^{K} c_{m k} \beta_{m i}\left|\boldsymbol{\phi}_{k}^{H} \boldsymbol{\phi}_{i}\right|^{2}\right. \\
&\left.\quad-\sum_{m=1}^{M} u_{m k}^{2} c_{m k}^{2} \beta_{m k^{\prime}}\left|\boldsymbol{\phi}_{k}^{H} \boldsymbol{\phi}_{k^{\prime}}\right|^{2}\right) \\
&=\sqrt{\tau p_{p}} q_{k^{\prime}} \sum_{m=1}^{M} u_{m k}^{2} c_{m k} \beta_{m k^{\prime}} \beta_{m k}-q_{k^{\prime}} \sum_{m=1}^{M} u_{m k}^{2} c_{m k}^{2} \beta_{m k^{\prime}} \\
&-\tau p_{p} q_{k^{\prime}} \sum_{m=1}^{M} u_{m k}^{2} c_{m k}^{2} \beta_{m k^{\prime}}\left|\boldsymbol{\phi}_{k}^{H} \boldsymbol{\phi}_{k^{\prime}}\right|^{2},
\end{aligned}
$$

where in the last step, we used equation (4). As a result, $C_{1}+C_{2}=\sqrt{\tau p_{p}} q_{k^{\prime}} \sum_{m=1}^{M} u_{m k}^{2} c_{m k} \beta_{m k^{\prime}} \beta_{m k}$. Then finally we have

$$
\begin{aligned}
\mathbb{E}\left\{\left|\mathrm{IUI}_{k k^{\prime}}\right|^{2}\right\}= & \rho q_{k^{\prime}}\left(\sum_{m=1}^{M} u_{m k}^{2} \beta_{m k^{\prime}} \gamma_{m k}\right) \\
& +\rho q_{k^{\prime}}\left|\boldsymbol{\phi}_{k}^{H} \boldsymbol{\phi}_{k^{\prime}}\right|^{2}\left(\sum_{m=1}^{M} u_{m k} \gamma_{m k} \frac{\beta_{m k^{\prime}}}{\beta_{m k}}\right)^{2} .
\end{aligned}
$$

The total noise for user $k$ is given by

$$
\mathbb{E}\left\{\left|\mathrm{TN}_{k}\right|^{2}\right\}=\mathbb{E}\left\{\left|\sum_{m=1}^{M} u_{m k} \hat{g}_{m k}^{*} n_{m}\right|^{2}\right\}=\sum_{m=1}^{M} u_{m k}^{2} \gamma_{m k},
$$

where the last equality is due to the fact that the terms $\hat{g}_{m k}$ and $n_{m}$ are uncorrelated. Finally, by substituting (39), (40), (49) and (50) into (9), SINR of $k$ th user is obtained by (10). which completes the proof of Theorem 1.

APPENDIX B

PROOF OF PROPOSITION 1

The standard form of GP is defined as follows [33]:

$P_{12}: \min f_{0}(\mathbf{x})$,

s.t. $f_{i}(\mathbf{x}) \leq 1, i=1, \cdots, m, g_{i}(\mathbf{x})=1, i=1, \cdots, p$,

where $f_{0}$ and $f_{i}$ are posynomial and $g_{i}$ are monomial functions. Moreover, $\mathbf{x}=\left\{x_{1}, \cdots, x_{n}\right\}$ represent the optimization variables. The SINR constraint in (15c) is not a posynomial function in its form, however it can be rewritten into the following posynomial function:

$$
\begin{array}{r}
\frac{\mathbf{u}_{k}^{H}\left(\sum_{k^{\prime} \neq k}^{K} q_{k^{\prime}}\left|\boldsymbol{\phi}_{k}^{H} \boldsymbol{\phi}_{k^{\prime}}\right|^{2} \boldsymbol{\Delta}_{k k^{\prime}} \boldsymbol{\Delta}_{k k^{\prime}}^{H}+\sum_{k^{\prime}=1}^{K} q_{k^{\prime}} \mathbf{D}_{k k^{\prime}}+\frac{1}{\rho} \mathbf{R}_{k}\right) \mathbf{u}_{k}}{\mathbf{u}_{k}^{H}\left(q_{k} \boldsymbol{\Gamma}_{k} \boldsymbol{\Gamma}_{k}^{H}\right) \mathbf{u}_{k}} \\
<\frac{1}{t}, \forall k .
\end{array}
$$

By applying a simple transformation, (52) is equivalent to the following inequality:

$$
q_{k}^{-1}\left(\sum_{k^{\prime} \neq k}^{K} a_{k k^{\prime}} q_{k^{\prime}}+\sum_{k^{\prime}=1}^{K} b_{k k^{\prime}} q_{k^{\prime}}+c_{k}\right)<\frac{1}{t},
$$

where

$$
\begin{aligned}
a_{k k^{\prime}} & =\frac{\mathbf{u}_{k}^{H}\left(\left|\boldsymbol{\phi}_{k}^{H} \boldsymbol{\phi}_{k^{\prime}}\right|^{2} \boldsymbol{\Delta}_{k k^{\prime}} \boldsymbol{\Delta}_{k k^{\prime}}^{H}\right) \mathbf{u}_{k}}{\mathbf{u}_{k}^{H}\left(\boldsymbol{\Gamma}_{k} \boldsymbol{\Gamma}_{k}^{H}\right) \mathbf{u}_{k}}, \\
b_{k k^{\prime}} & =\frac{\mathbf{u}_{k}^{H} \mathbf{D}_{k k^{\prime}} \mathbf{u}_{k}}{\mathbf{u}_{k}^{H}\left(\boldsymbol{\Gamma}_{k} \boldsymbol{\Gamma}_{k}^{H}\right) \mathbf{u}_{k}}, c_{k}=\frac{\mathbf{u}_{k}^{H} \mathbf{R}_{k} \mathbf{u}_{k}}{\rho \mathbf{u}_{k}^{H}\left(\boldsymbol{\Gamma}_{k} \boldsymbol{\Gamma}_{k}^{H}\right) \mathbf{u}_{k}} .
\end{aligned}
$$

The transformation in (53) shows that the left-hand side of (52) is a posynomial function. Therefore, the power allocation problem $P_{4}$ is a standard GP (convex problem), where the objective function and constraints are monomial and posynomial, respectively, which completes the proof of Proposition 1.

\section{APPENDIX C}

\section{PROOF OF LEMMA 1}

This lemma is proven by exploiting the unique optimal solution of uplink max-min SINR problem with total power through an eigensystem [22]. This problem is iteratively solved and the optimal receiver filter coefficient $\tilde{\mathbf{U}}$ is determined by solving Problem $P_{3}$. Next, we scale the power allocation at 


$$
\operatorname{SINR}_{k}^{\mathrm{UP}}=\frac{q_{k} \mathbf{u}_{k}^{H}(\overbrace{\boldsymbol{\Gamma}_{k} \boldsymbol{\Gamma}_{k}^{H}}^{\tilde{\mathbf{D}}_{k}}) \mathbf{u}_{k}}{\mathbf{u}_{k}^{H}(\sum_{k^{\prime} \neq k}^{K} q_{k^{\prime}} \underbrace{\left|\boldsymbol{\phi}_{k}^{H} \boldsymbol{\phi}_{k^{\prime}}\right|^{2} \boldsymbol{\Delta}_{k k^{\prime}} \boldsymbol{\Delta}_{k k^{\prime}}^{H}}_{\tilde{\mathbf{R}}_{k k^{\prime}}}+\sum_{k^{\prime}=1}^{K} q_{k^{\prime}} \underbrace{\mathbf{D}_{k k^{\prime}}}_{\tilde{\tilde{\mathbf{R}}}_{k k^{\prime}}}+\frac{1}{\rho} \mathbf{R}_{k}) \mathbf{u}_{k}} .
$$

each user such that the per-user power constraints are satisfied. Let us consider the following optimization problem for a given receiver filter coefficient $\tilde{\mathbf{U}}$ :

$$
P_{11}: C_{k}^{\mathrm{UP}}\left(\tilde{\mathbf{U}}, P_{\text {tot }}\right)=\max _{q_{k}} \min _{k=1, \cdots, K} \operatorname{SINR}_{k}^{\mathrm{UP}}(\tilde{\mathbf{U}}, \mathbf{q}),
$$

$$
\text { subject to } \sum_{k=1}^{K} q_{k} \leq P_{\text {tot }} \text {. }
$$

The optimal solution of Problem $P_{11}$ can be determined by finding the unique eigenvector of an eigensystem and the power allocation $\tilde{\mathbf{q}}$ satisfies the condition $\sum_{k=1}^{K} \tilde{q}_{k}=P_{\text {tot }}$ [22]. The SINRs of all users defined in (10), can be collectively written as

$$
\tilde{\mathbf{q}} \frac{1}{C_{k}^{\mathrm{UP}}\left(\tilde{\mathbf{U}}, P_{\mathrm{tot}}\right)}=\mathbf{D} \Psi(\tilde{\mathbf{U}}) \tilde{\mathbf{q}}+\mathbf{D} \boldsymbol{\sigma}(\tilde{\mathbf{U}})
$$

where $\boldsymbol{\sigma}(\tilde{\mathbf{U}}) \in \mathbb{C}^{K \times 1}, \sigma_{k}\left(\mathbf{u}_{k}\right)=\frac{1}{\rho} \sum_{m=1}^{M} \tilde{u}_{m k} \gamma_{m k}$ and $\mathbf{D}$ and $\boldsymbol{\Psi}(\tilde{\mathbf{U}})$ are defined as

$$
\begin{array}{r}
\mathbf{D}=\operatorname{diag}\left[\frac{1}{\tilde{\mathbf{u}}_{1}^{H} \tilde{\mathbf{D}}_{1} \tilde{\mathbf{u}}_{1}}, \cdots, \frac{1}{\tilde{\mathbf{u}}_{K}^{H} \tilde{\mathbf{D}}_{K} \tilde{\mathbf{u}}_{K}}\right], \\
{[\Psi(\tilde{\mathbf{U}})]_{k k^{\prime}}=\left\{\begin{array}{l}
\tilde{\mathbf{u}}_{k}^{H} \tilde{\tilde{\mathbf{R}}}_{k k} \tilde{\mathbf{u}}_{k}, \\
\tilde{\mathbf{u}}_{k}^{H} \tilde{\mathbf{R}}_{k k^{\prime}} \tilde{\mathbf{u}}_{k}+\tilde{\mathbf{u}}_{k}^{H} \tilde{\tilde{\mathbf{R}}}_{k k^{\prime}} \tilde{\mathbf{u}}_{k}, k \neq k^{\prime},
\end{array}\right.}
\end{array}
$$

where using (10), $\tilde{\mathbf{D}}_{k} \tilde{\mathbf{R}}_{k k^{\prime}}$ and $\tilde{\tilde{\mathbf{R}}}_{k k^{\prime}}$ are defined as (58), shown at the top of this page.

Having both sides of (56) multiplied by $\mathbf{1}^{T}=[1, \cdots, 1]^{T}$, we obtain $\frac{1}{C_{k}^{\mathrm{UP}}\left(\tilde{\mathbf{U}}, P_{\text {tot }}\right)}=\frac{1}{P_{\text {tot }}} \mathbf{1}^{T} \tilde{\mathbf{D}} \Psi(\tilde{\mathbf{U}}) \tilde{\mathbf{q}}+\frac{1}{P_{\text {tot }}} \mathbf{1}^{T} \mathbf{D} \boldsymbol{\sigma}(\tilde{\mathbf{U}})$, which can be combined with (56) to define the following eigensystem:

$$
\boldsymbol{\Lambda}\left(\tilde{\mathbf{U}}, P_{\mathrm{tot}}\right) \tilde{\mathbf{q}}_{\mathrm{ext}}=\frac{1}{C_{k}^{\mathrm{UP}}\left(\tilde{\mathbf{U}}, P_{\mathrm{tot}}\right)} \tilde{\mathbf{q}}_{\mathrm{ext}}, \quad\left[\tilde{\mathbf{q}}_{\mathrm{ext}}\right]_{K+1}=1,
$$

where the extended coupling matrix $\boldsymbol{\Lambda}\left(\tilde{\mathbf{D}}, P_{\text {tot }}\right)$ is given by

$$
\boldsymbol{\Lambda}\left(\tilde{\mathbf{D}}, P_{\mathrm{tot}}\right)=\left[\begin{array}{cc}
\mathbf{D} \boldsymbol{\Psi}^{T}(\tilde{\mathbf{U}}) & \mathbf{D} \boldsymbol{\sigma}(\tilde{\mathbf{U}}) \\
\frac{1}{P_{\mathrm{tot}}} \mathbf{1}^{T} \mathbf{D} \Psi^{T}(\tilde{\mathbf{U}}) & \frac{1}{P_{\mathrm{tot}}} \mathbf{1}^{T} \mathbf{D} \boldsymbol{\sigma}(\tilde{\mathbf{U}})
\end{array}\right] .
$$

The optimal power allocation $\tilde{\mathbf{q}}$ is obtained by determining the eigenvector corresponding to the maximum eigenvalue of $\Lambda\left(\tilde{\mathbf{U}}, P_{\text {tot }}\right)$ and scaling the last element to one as follows:

$$
\tilde{\mathbf{q}}_{\mathrm{ext}}=\left[\begin{array}{c}
\tilde{\mathbf{q}} \\
1
\end{array}\right], \boldsymbol{\Lambda}\left(\tilde{\mathbf{U}}, P_{\mathrm{tot}}\right) \tilde{\mathbf{q}}_{\mathrm{ext}}=\lambda_{\max }\left(\boldsymbol{\Lambda}\left(\tilde{\mathbf{U}}, P_{\mathrm{tot}}\right)\right) \tilde{\mathbf{q}}_{\mathrm{ext}}
$$

Note that the dominant eigenvector can be scaled by any positive value to satisfy a particular condition. As such, we further scale $\tilde{\mathbf{q}}$ to satisfy the per-user power constraints as follows:

$$
\tilde{\mathbf{q}}=\left[\begin{array}{c}
\frac{\hat{q}_{1}}{\max (\hat{\mathbf{q}})} \\
\vdots \\
\frac{\hat{q}_{K}}{\max (\hat{\mathbf{q}})}
\end{array}\right], \text { where } \hat{\mathbf{q}}=\left[\begin{array}{c}
\tilde{q}_{1} \\
p_{\max }^{(1)} \\
\vdots \\
\tilde{q}_{K} \\
p_{\max }^{(K)}
\end{array}\right],
$$

where first the ratios between each component of the allocated power, $\tilde{q}_{k}, \forall k$, and the maximum available power, $p_{\max }^{(k)}, \forall k$, are calculated. Then the power allocation $\tilde{\mathbf{q}}$ is obtained by dividing all components of $\tilde{\mathbf{q}}$ by the maximum value among the components of $\hat{\mathbf{q}}$, i.e., $\max (\hat{\mathbf{q}})$. In the next iteration, the same max-min problem is solved with a new total power constraint obtained by summing up the allocated power to all users in the previous iteration, i.e., $P_{\text {tot }}=\sum_{k=1}^{K} \tilde{q}_{k}$. At the convergence, the per-user power constraints are satisfied with achieving the same uplink SINR for all users. Interestingly, if this max-min problem is solved with the corresponding total power constraint, then it will converge to the same optimal solution of max-min problem with per-user power constraints. This is due to the property that the eigensystem exploited to obtain the power allocation in (58) has a unique positive eigenvalue and a corresponding unique eigenvector. Therefore, Problems $P_{1}$ and $P_{5}$ are equivalent and have the same optimal solution.

\section{APPENDIX D PROOF OF THEOREM 3}

To achieve the same SINR tuples in both the uplink and the downlink, the following condition should be satisfied:

$$
\operatorname{SINR}_{k}^{\mathrm{DL}}(\mathbf{U}, \mathbf{p})=\operatorname{SINR}_{k}^{\mathrm{UP}}(\mathbf{U}, \mathbf{q}), \forall k .
$$

By substituting uplink and downlink SINRs, in (19) and (18), respectively, in equation (62) and summing all equations by both sides, we have

$$
p_{1} \sum_{m=1}^{M} u_{m 1}^{2} \gamma_{m 1}+\cdots+p_{K} \sum_{m=1}^{M} u_{m K}^{2} \gamma_{m K}=\sum_{k=1}^{K} q_{k}
$$

Therefore, this condition between the total transmit power on the uplink and the equivalent total transmit power on the downlink should be satisfied to realize the same SINRs for all set of users, which completes the proof of Theorem 3.

\section{REFERENCES}

[1] F. Boccardi, R. W. Heath, A. Lozano, T. L. Marzetta, and P. Popovski, "Five disruptive technology directions for 5G," IEEE Commun. Mag., vol. 52, no. 2, pp. 74-80, Feb. 2014. 
[2] A. Zappone, L. Sanguinetti, G. Bacci, E. Jorswieck, and M. Debbah, "Energy-efficient power control: A look at 5G wireless technologies," IEEE Trans. Signal Process., vol. 64, no. 7, pp. 1668-1683, Apr. 2016

[3] E. Björnson, E. A. Jorswieck, M. Debbah, and B. Ottersten, "Multiobjective signal processing optimization: The way to balance conflicting metrics in 5G systems," IEEE Signal Process. Mag., vol. 31, no. 6, pp. 14-23, Nov. 2014.

[4] H. Q. Ngo, A. Ashikhmin, H. Yang, E. G. Larsson, and T. L. Marzetta, "Cell-free massive MIMO versus small cells," IEEE Trans. Wireless Commun., vol. 16, no. 3, pp. 1834-1850, Mar. 2017.

[5] M. Bashar, K. Cumanan, A. G. Burr, M. Debbah, and H. Q. Ngo, "Enhanced max-min SINR for uplink cell-free massive MIMO systems," in Proc. IEEE ICC, May 2018, pp. 1-7.

[6] E. Nayebi, A. Ashikhmin, T. L. Marzetta, H. Yang, and B. D. Rao, "Precoding and power optimization in cell-free massive MIMO systems," IEEE Trans. Wireless Commun., vol. 16, no. 7, pp. 4445-4459, Jul. 2017.

[7] G. Interdonato, H. Q. Ngo, E. G. Larsson, and P. Frenger, "On the performance of cell-free massive MIMO with short-term power constraints," in Proc. IEEE CAMAD, Oct. 2016, pp. 1-6.

[8] S. Buzzi and C. D'Andrea, "Cell-free massive MIMO: User-centric approach," IEEE Wireless Commun. Lett., vol. 6, no. 6, pp. 706-709, Dec. 2017

[9] M. K. Karakayali, G. J. Foschini, and R. A. Valenzuela, "Network coordination for spectrally efficient communications in cellular systems," IEEE Wireless Commun., vol. 13, no. 4, pp. 56-61, Aug. 2006.

[10] E. Björnson, R. Zakhour, D. Gesbert, and B. Ottersten, "Cooperative multicell precoding: Rate region characterization and distributed strategies with instantaneous and statistical CSI," IEEE Trans. Signal Process., vol. 58, no. 8, pp. 4298-4310, Aug. 2010.

[11] S.-H. Park, O. Simeone, O. Sahin, and S. Shamai (Shitz), "Fronthaul compression for cloud radio access networks: Signal processing advances inspired by network information theory," IEEE Signal Process. Mag., vol. 31, no. 6, pp. 69-79, Nov. 2014.

[12] S.-H. Park, O. Simeone, and S. Shamai (Shitz), "Joint optimization of cloud and edge processing for fog radio access networks," IEEE Trans. Wireless Commun., vol. 15, no. 11, pp. 7621-7632, Nov. 2016.

[13] H. Q. Ngo, L.-N. Tran, T. Q. Duong, M. Matthaiou, and E. G. Larsson, "On the total energy efficiency of cell-free massive MIMO," IEEE Trans. Green Commun. Netw., vol. 2, no. 1, pp. 25-39, Mar. 2017.

[14] K. Cumanan, R. Krishna, L. Musavian, and S. Lambotharan, "Joint beamforming and user maximization techniques for cognitive radio networks based on branch and bound method," IEEE Trans. Wireless Commun., vol. 9, no. 10, pp. 3082-3092, Oct. 2010.

[15] K. Cumanan, L. Musavian, S. Lambotharan, and A. B. Gershman, "SINR balancing technique for downlink beamforming in cognitive radio networks," IEEE Signal Process. Lett., vol. 17, no. 2, pp. 133-136, Feb. 2010 .

[16] K. Cumanan, J. Tang, and S. Lambotharan, "Rate balancing based linear transceiver design for multiuser MIMO system with multiple linear transmit covariance constraints," in Proc. IEEE ICC, Jun. 2011, pp. 1-5.

[17] A. Wiesel, Y. C. Eldar, and S. Shamai (Shitz), "Linear precoding via conic optimization for fixed MIMO receivers," IEEE Trans. Signal Process., vol. 54, no. 1, pp. 161-176, Jan. 2006.

[18] D. W. H. Cai, T. Q. S. Quek, and C. W. Tan, "A unified analysis of maxmin weighted SINR for MIMO downlink system," IEEE Trans. Signal Process., vol. 59, no. 8, pp. 3850-3862, Aug. 2011.

[19] G. Golub and C. F. Van Loan, Matrix Computations, 2nd ed. Baltimore, MD, USA: The Johns Hopkins Univ. Press, 1996.

[20] S. P. Boyd, S.-J. Kim, A. Hassibi, and L. Vandenberghe, "A tutorial on geometric programming," Optim. Eng., vol. 8, no. 1, pp. 67-128, Apr. 2007.

[21] M. Chiang, C. W. Tan, D. P. Palomar, D. O'Neill, and D. Julian, "Power control by geometric programming," in Resource Allocation in Next Generation Wireless Networks. W. Li and Y. Pan, Eds. Commack, NY, USA: Nova, 2006.

[22] M. Schubert and H. Boche, "Solution of the multiuser downlink beamforming problem with individual SINR constraints," IEEE Trans. Veh. Technol., vol. 53, no. 1, pp. 18-28, Jan. 2004.

[23] M. Schubert and H. Boche, "Iterative multiuser uplink and downlink beamforming under SINR constraints," IEEE Trans. Signal Process. vol. 53, no. 7, pp. 2324-2334, Jul. 2005.

[24] D. N. C. Tse and P. Viswanath, "Downlink-uplink duality and effective bandwidths," in Proc. IEEE ISIT, Jun./Jul. 2002, p. 52.
[25] M. Chiang, P. Hande, T. Lan, and C. W. Tan, "Power control in wireless cellular networks," in Foundation and Trends in Networking. Boston, MA, USA: Now, 2007.

[26] H. Ahmadi, A. Farhang, N. Marchetti, and A. MacKenzie, "A game theoretic approach for pilot contamination avoidance in massive MIMO," IEEE Wireless Commun. Lett., vol. 5, no. 1, pp. 12-15, Feb. 2016.

[27] M. A. Imran, S. A. R. Zaidi, and Z. Shakir, Access, Fronthaul and Backhaul Networks for $5 G$ \& Beyond. Edison, NJ, USA: Institution of Engineering and Technology, 2017.

[28] M. Bashar, K. Cumanan, A. G. Burr, H. Q. Ngo, and M. Debbah, "Cell-free massive MIMO with limited backhaul," in Proc. IEEE ICC, May 2018, pp. 1-7.

[29] M. Bashar, K. Cumanan, A. G. Burr, H. Q. Ngo, and M. Debbah "Maxmin SINR of cell-free Massive MIMO uplink with optimal uniform quantization," IEEE Trans. Commun., submitted for publication.

[30] A. Burr, M. Bashar, and D. Maryopi, "Cooperative access networks: Optimum fronthaul quantization in distributed massive MIMO and cloud RAN," in Proc. IEEE VTC, Jun. 2018, pp. 1-7.

[31] A. J. Fehske, P. Marsch, and G. P. Fettweis, "Bit per Joule efficiency of cooperating base stations in cellular networks," in Proc. IEEE GLOBECOM Workshops (GC Wkshps), Dec. 2010, pp. 1406-1411.

[32] F. Rashid-Farrokhi, K. J. R. Liu, and L. Tassiulas, "Transmit beamforming and power control for cellular wireless systems," IEEE J. Sel. Area Commun., vol. 16, no. 8, pp. 1437-1450, Oct. 1998.

[33] S. Boyd and L. Vandenberghe, Convex Optimization. Cambridge, U.K.: Cambridge Univ. Press, 2004.

[34] C.-C. Chien, H.-J. Su, and H.-J. Li, "Joint beamforming and power allocation for MIMO relay broadcast channel with individual SINR constraints," IEEE Trans. Veh. Technol., vol. 63, no. 4, pp. 1660-1677, May 2004.

[35] G. H. Golub and C. F. Van Loan, Matrix Computations, 3rd ed. Baltimore, MD, USA: The Johns Hopkins Univ. Press, 1996.

[36] Y. Nesterov and A. Nemirovskii, Interior-Point Polynomial Algorithms in Convex Programming (Studies in Applied Mathematics). Philadelphia, PA, USA: SIAM, 1994

[37] M. S. Lobo, L. Vandenberghe, S. Boyd, and H. Lebret, "Applications of second-order cone programming," Linear Algebra Appl., vol. 284 nos. 1-3, pp. 193-228, Nov. 1998

[38] J. F. Sturm, "Using SeDuMi 1.02, a MATLAB toolbox for optimization over symmetric cones," Optim. Methods Softw., vol. 11, nos. 1-4, pp. $625-653,1999$.

[39] A. Tang, J. Sun, and K. Gong, "Mobile propagation loss with a low base station antenna for NLOS street microcells in urban area," in Proc. IEEE VTC, May 2001, pp. 333-336.

[40] F. Rusek et al., "Scaling up MIMO: Opportunities and challenges with very large arrays," IEEE Signal Process. Mag., vol. 30, no. 1, pp. 40-60, Jan. 2013.

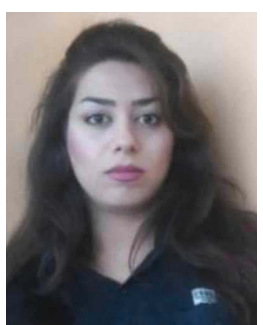

Manijeh Bashar received the B.Sc. degree in electrical engineering from the University of Guilan, Rasht, Iran, in 2009, and the M.Sc. degree in communication systems engineering from the Shiraz University of Technology, Shiraz, Iran, in 2013 She is currently pursuing the Ph.D. degree with the University of York, U.K. She is currently a Research Fellow with the Fifth Generation (5G) Innovation Centre, University of Surrey, U.K.

In 2017, she was an Academic Visitor with the Department of Electronics and Nanoengineering, Aalto University, Espoo, Finland. Her current research interests include cooperative communications for $5 \mathrm{G}$ networks, including distributed massive multiple input multiple output (MIMO), Cloud-RAN, Fog-RAN, nonorthogonal multiple access, network slicing, convex optimization techniques, and millimeter-wave channel modeling. She received the Short Term Scientific Mission Scholarship Award from European COST-IC1004 "Cooperative Radio Communications for Green Smart Environments." She received the First Place (based on jury) at the IEEE WCNC 2018 3-min Ph.D. Thesis Competition for her research in cell-free massive MIMO. 


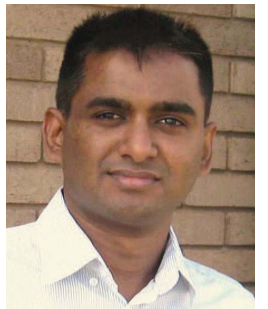

Kanapathippillai Cumanan received the B.Sc degree (Hons.) in electrical and electronic engineering from the University of Peradeniya, Sri Lanka, in 2006, and the Ph.D. degree in signal processing for wireless communications from Loughborough University, Loughborough, U.K., in 2009.

In 2006, he was a Teaching Assistant with the Department of Electrical and Electronic Engineering, University of Peradeniya. In 2011, he was an Academic Visitor with the Department of Electrical and Computer Engineering, National University of Singapore, Singapore. He was with the School of Electronic, Electrical and System Engineering, Loughborough University. From 2012 to 2014, he was a Research Associate with the School of Electrical and Electronic Engineering, Newcastle University, U.K. He is currently a Lecturer with the Department of Electronic Engineering, University of York, U.K. His research interests include non-orthogonal multiple access, massive multiple input multiple output, physical-layer security, cognitive radio networks, convex optimization techniques, and resource allocation techniques.

Between 2006 and 2007, he was a Research Student with Cardiff University, U.K. He received the Overseas Research Student Award Scheme from Cardiff University.

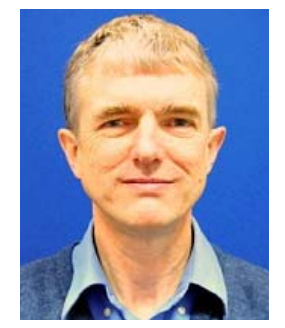

Alister G. Burr was born in London, U.K., in 1957. He received the B.Sc. degree in electronic engineering from The University of Southampton, Southampton, U.K., in 1979, and the Ph.D. degree from the University of Bristol, Bristol, U.K., in 1984. Between 1975 and 1985, he was with the Thorn-EMI Central Research Laboratories, London. In 1985, he joined the Department of Electronics (currently the Department of Electronic Engineering), University of York, York, U.K., where he has been a Professor of communications since 2000. He has authored or co-authored around 250 papers in refereed international conferences and journals. He has authored the book entitled Modulation and Coding for Wireless Communications (Englewood Cliffs, NJ, USA: PrenticeHall/ PHEI, 2001), and has co-authored the book entitled Wireless PhysicalLayer Network Coding (Cambridge, U.K.: Cambridge Univ. Press, 2018). His research interests include wireless communication systems, especially multiple input multiple output, cooperative systems, physical-layer network coding, and iterative detection and decoding techniques. He received the Senior Research Fellowship by the U.K. Royal Society in 1999 and the J. Langham Thompson Premium from the Institution of Electrical Engineers in 2002. He has also given more than 15 invited presentations, including three keynote presentations. He was the Chair, working group 2, of a series of European COST Programs, including IC1004 "Cooperative Radio Communications for Green Smart Environments" (which have been influential in 3GPP standardization). He was an Associate Editor of IEEE COMMUNICATIONS LETTERS, the Workshop Chair of the 2016 IEEE International Conference on Communications, and the Technical Program Committee Co-Chair of the 2018 Annual IEEE International Symposium on Personal, Indoor and Mobile Radio Communications.

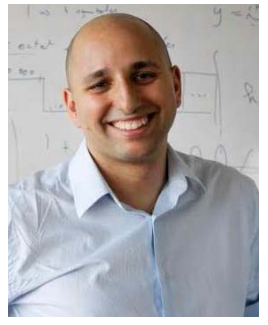

Mérouane Debbah received the M.Sc. and $\mathrm{Ph} . \mathrm{D}$. degrees from the Ecole Normale Supérieure Paris-Saclay, France. In 1996, he joined the Ecole Normale Supérieure Paris-Saclay. He was with Motorola Labs, Saclay, France, from 1999 to 2002, and also with the Vienna Research Center for Telecommunications, Vienna, Austria, until 2003. From 2003 to 2007, he was an Assistant Professor with the Mobile Communications Department, Institut Eurecom, Sophia Antipolis, France. From 2007 to 2014, he was the Director of the Alcatel-Lucent Chair on Flexible Radio. Since 2007, he has been a Full Professor with CentraleSupelec, Gif-sur-Yvette, France. Since 2014, he has been a Vice-President of the Huawei France Research and Development Center and the Director of the Mathematical and Algorithmic Sciences Lab. He has managed eight EU projects and more than 24 national and international projects. His research interests lie in fundamental mathematics, algorithms, statistics, information, and communication sciences research. He is an IEEE Fellow, a WWRF Fellow, and a Membre émérite SEE. He was a recipient of the ERC Grant MORE (Advanced Mathematical Tools for Complex Network Engineering). He was a recipient of the Mario Boella Award in 2005, the IEEE Glavieux Prize Award in 2011, and the Qualcomm Innovation Prize Award in 2012. He received 19 best paper awards, among which the 2007 IEEE GLOBECOM Best Paper Award, the Wi-Opt 2009 Best Paper Award, the 2010 Newcom++ Best Paper Award, the WUN CogCom Best Paper 2012 and 2013 Award, the 2014 WCNC Best Paper Award, the 2015 ICC Best Paper Award, the 2015 IEEE Communications Society Leonard G. Abraham Prize, the 2015 IEEE Communications Society Fred W. Ellersick Prize, the 2016 IEEE Communications Society Best Tutorial Paper Award, the 2016 European Wireless Best Paper Award, the 2017 Eurasip Best Paper Award, the 2018 IEEE Marconi Prize Paper Award, and the Valuetools 2007, Valuetools 2008, CrownCom 2009 , Valuetools 2012, SAM 2014, and 2017 IEEE Sweden VT-COM-IT Joint Chapter best student paper awards. He is an Associate Editor-in-Chief of the journal Random Matrix: Theory and Applications. He was an Associate Area Editor and Senior Area Editor of the IEEE TRANSACTIONS ON SIGNAL PROCESSING from 2011 to 2013 and from 2013 to 2014, respectively.

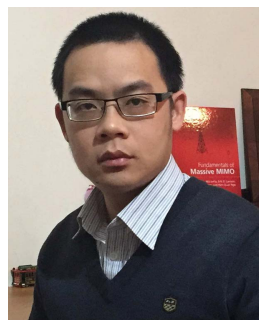

Hien Quoc Ngo received the B.S. degree in electrical engineering from the Ho Chi Minh City University of Technology, Vietnam, in 2007, the M.S. degree in electronics and radio engineering from Kyung Hee University, South Korea, in 2010, and the Ph.D. degree in communication systems from Linköping University (LiU), Sweden, in 2015. In 2014, he visited the Nokia Bell Labs, Murray Hill, NY, USA. From 2016 to 2017, he was a VR Researcher with the Department of Electrical Engineering (ISY), LiU. He was also a Visiting Research Fellow with the School of Electronics, Electrical Engineering and Computer Science, Queen's University Belfast, U.K., funded by the Swedish Research Council.

$\mathrm{He}$ is currently a Lecturer with Queen's University Belfast. He has co-authored many research papers in wireless communications and the textbook Fundamentals of Massive MIMO (Cambridge University Press, 2016). His main research interests include massive (large-scale) multipleinput multiple-output (MIMO) systems, cell-free massive MIMO, physicallayer security, and cooperative communications.

Dr. Ngo was a recipient of the IEEE ComSoc Stephen O. Rice Prize in Communications Theory in 2015, the IEEE ComSoc Leonard G. Abraham Prize in 2017, and the IEEE Sweden VT-COM-IT Joint Chapter Best Student Journal Paper Award in 2015. He received the Best Ph.D. Award from EURASIP in 2018. He was an IEEE Communications Letters Exemplary Reviewer for 2014, an IEEE Transactions on Communications Exemplary Reviewer for 2015, and an IEEE Wireless Communications Letters Exemplary Reviewer for 2016. He currently serves as an Editor for IEEE Wireless Communications Letters, Digital Signal Processing, and the IEICE Transactions on Fundamentals of Electronics, Communications and Computer Sciences. He was a Guest Editor of the IET Communications, special issue on "Recent Advances on 5G Communications" and the IEEE ACCESS, special issue on "Modelling, Analysis, and Design of 5G Ultra-Dense Networks," in 2017. He has been a member of Technical Program Committees for several IEEE conferences, such as ICC, GLOBECOM, WCNC, and VTC. 


\title{
On the Uplink Max-Min SINR of Cell-Free Massive MIMO Systems
}

\author{
Manijeh Bashar ${ }^{\circledR}$, Student Member, IEEE, Kanapathippillai Cumanan ${ }^{\circledR}$, Member, IEEE, \\ Alister G. Burr ${ }^{\circledR}$, Senior Member, IEEE, Mérouane Debbah, Fellow, IEEE, \\ and Hien Quoc Ngo ${ }^{\mathbb{D}}$, Member, IEEE
}

\begin{abstract}
A cell-free massive multiple-input multiple-output system is considered using a max-min approach to maximize the minimum user rate with per-user power constraints. First, an approximated uplink user rate is derived based on channel statistics. Then, the original max-min signal-to-interferenceplus-noise ratio problem is formulated for the optimization of receiver filter coefficients at a central processing unit and user power allocation. To solve this max-min non-convex problem, we decouple the original problem into two sub-problems, namely, receiver filter coefficient design and power allocation. The receiver filter coefficient design is formulated as a generalized Eigenvalue problem, whereas the geometric programming (GP) is used to solve the user power allocation problem. Based on these two sub-problems, an iterative algorithm is proposed, in which both problems are alternately solved while one of the design variables is fixed. This iterative algorithm obtains a globally optimum solution, whose optimality is proved through establishing an uplink-downlink duality. Moreover, we present a novel sub-optimal scheme which provides a GP formulation to efficiently and globally maximize the minimum uplink user rate. The numerical results demonstrate that the proposed scheme substantially outperforms the existing schemes in the literature.
\end{abstract}

Index Terms-Cell-free massive MIMO, max-min resource allocation, geometric programming, uplink-downlink duality, convex optimization, generalized eigenvalue problem.

Manuscript received November 20, 2017; revised May 11, 2018 and August 12, 2018; accepted December 17, 2018. The research described in this paper was supported in part by the European Horizon 2020 Programme under MCSA-ITN-2016-SPOTLIGHT (Grant 722788) and MSCA-RISE-2015-ATOM (Grant 690750), and in part through a University of York scholarship. This work was also supported in part by the European Commission under the 5GPPP project 5GXcast (H2020-ICT-2016-2 call, grant number 761498). The views expressed in this contribution are those of the authors and do not necessarily represent the project. The associate editor coordinating the review of this paper and approving it for publication was B. Natarajan. (Corresponding author: Manijeh Bashar.)

M. Bashar is with the Department of Electronic Engineering, University of York, York YO10 5DD, U.K., and also with the 5G Innovation Centre, Institute for Communication Systems, University of Surrey, Guildford GU2 7XH, U.K. (e-mail: mb1465@york.uc.ak; m.bashar@ surrey.ac.uk).

K. Cumanan and A. G. Burr are with the Department of Electronic Engineering, University of York, York YO10 5DD, U.K. (e-mail: kanapathippillai.cumanan@york.ac.uk; alister.burr@york.ac.uk).

M. Debbah is with the Large Networks and Systems Group, CentraleSupelec, Universite Paris-Saclay, 91192 Gif-sur-Yvette, France, and also with the Mathematical and Algorithmic Sciences Lab, Huawei Technologies Co., Ltd., 92100 Boulogne-Billancourt, France (e-mail: merouane.debbah@ centralesupelec.fr).

H. Q. Ngo is with the School of Electronics, Electrical Engineering and Computer Science, Queen's University Belfast, Belfast BT3 9DT, U.K. (e-mail: hien.ngo@qub.ac.uk).

Color versions of one or more of the figures in this paper are available online at http://ieeexplore.iee.org.

Digital Object Identifier 10.1109/TWC.2019.2892463

\section{INTRODUCTION}

$\mathbf{F}$ UTURE fifth generation (5G) wireless communication networks will deliver a wide range of new user services and dramatically increased data rates. Massive multiple-input multiple-output (MIMO) has been recognized as one of the key elements of 5G systems, due to its potential for extremely high spectral efficiency [1]-[3]. This paper considers cell-free Massive MIMO which has received much attention recently because of its potential to ensure uniformly good service throughput for all users [4]-[8]. Cell-free Massive MIMO is a combination of distributed MIMO and Massive MIMO, and there is no cell boundary [4]. It is a scalable version of network MIMO which is also called coordinated multipoint processing (CoMP) [9], [10]. The distributed access points (APs) are connected to a central processing unit (CPU) via high capacity backhaul links [4]. Cell-free Massive MIMO is thus also a scalable version of the cloud radio access network (CRAN). In CRAN, there are heavy communication burdens on the backhaul, and computation burdens on the CPU, as all signal processing is performed at the CPU [11]. The fog radio access network (FRAN) [12] can overcome some of the problems of CRAN. It moves some signal processing functionalities from the CPU back to the AP, where in this case the APs can also perform part of the signal processing. Hence, the tasks required of the CPU can also be reduced. The more processing is moved to the AP, the less is the burden imposed on the CPU.

In [4], [6], and [13], the authors propose that the APs design the linear receivers based on the estimated channels, and that this is carried out locally at the APs. Hence, the CPU exploits only the statistics of the channel for data detection. However, in this paper, we propose to exploit a new receiver filter at the CPU to improve the performance of cell-free Massive MIMO systems. The coefficients of the proposed receiver filter are designed based on only the statistics of the channel, which is different from the linear receiver at the APs. The proposed receiver filter provides more freedom in the design parameters, and hence significantly improves the performance of the uplink of cell-free Massive MIMO. In other words, the receiver filter coefficients are designed after exploiting linear detection at the CPU. Therefore, the uplink problem in the present paper is different from the problem studied in [4], as in [4], the authors do not consider the receiver filter coefficients. 
In this paper, we investigate an uplink max-min signalto-interference-plus-noise ratio (SINR) problem in a cell-free Massive MIMO system. In particular, we propose a new approach to solve this max-min problem. A similar max-min SINR problem based on SINR known as SINR balancing in the literature has been considered for cognitive radio networks in [14]-[16]. In [17] and [18], the authors consider MIMO systems and study the problem of max-min user SINR to maximize the smallest user SINR. Note that the same maxmin problem is investigated in an uplink cell-free Massive MIMO systems in [4] where user power allocation is utilized by using a bisection search approach. However, the maxmin SINR problem considered in this paper is different from the scheme in [4] due to the design parameters (in terms of receiver filter coefficients and user power allocation) and solution approach. In particular, the receiver filter coefficients and power allocation are optimized in the proposed approach whereas the work in [4] only considered user power allocations. First, we derive the average SINR of the user by incorporating a matched filtering receiver and formulate the corresponding max-min SINR problem. This original maxmin problem in terms of receiver filter coefficients and power allocations is not jointly convex. To circumvent this nonconvexity issue, we decompose the original problem into two sub-problems, namely, receiver filter coefficient design, and power allocation. It is shown that the receiver filter coefficient design problem can be solved through a generalized eigenvalue problem [19] whereas the user power allocation problems can be formulated using standard geometric programming (GP) [20], [21]. An iterative procedure is proposed whereby at each iteration, one of the sub-problems is solved while the other design variable is fixed. To validate the optimality of the proposed scheme, we show that there exists an equivalent downlink problem to realize the same user rate in the uplink with an equivalent total power constraint and the same receiver filter coefficients. By solving this equivalent problem, the optimality of the proposed scheme in the uplink is proved. The problem of uplink-downlink duality has been investigated in [17] and [22]-[25]. Simulation results are provided to demonstrate the performance of the proposed scheme which confirms that the proposed scheme outperforms the scheme in [4] in terms of achieved user rate. In addition, we propose a new sub-optimal max-min SINR scheme using a GP formulation which does not require any iterative approach as in [4]. The contributions and results are as follows:

1. To improve the performance of the system, we propose to use a novel receiver filter, operating at the CPU, which can be designed based only on the statistics of the channel. Note that this is different from the linear matched filtering receiver in [4].

2. The uplink user throughput using the proposed filter is derived based on channel statistics and taking into account the effects of channel estimation errors and the effect of pilot sequences. We propose a novel approach to solve the uplink max-min SINR problem, decoupling the original problem into two sub-problems, which are solved using an iterative algorithm. These sub-problems are

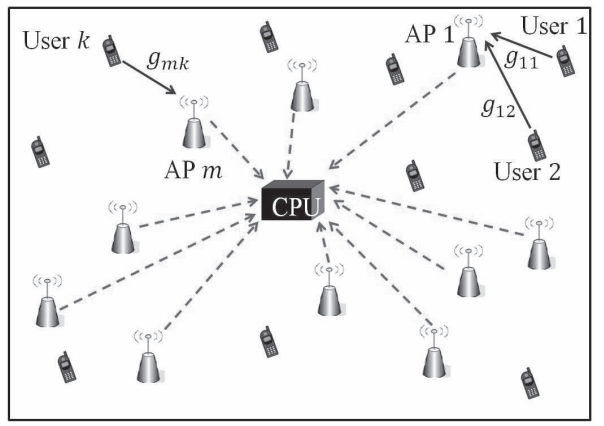

Fig. 1. The uplink of a cell-free Massive MIMO system with $K$ singleantenna users and $M$ APs. The solid lines denote the uplink channels and the dashed lines present the backhaul links from the APs to the CPU.

formulated as GP and a generalized eigenvalue problem, and both sub-problems are solved at each iteration.

3. We prove that the proposed iterative algorithm provides the globally optimal solution for the original non-convex max-min SINR problem. The optimality of the proposed algorithm is proved through establishing the uplinkdownlink duality for cell-free Massive MIMO.

4. We present a sub-optimal max-min SINR scheme by formulating it into a standard GP which does not require an iterative approach and shows the same performance as in [4].

5. We present the complexity analysis of different schemes.

6. We present numerical results supporting the convergence analysis and the theoretical derivations of the optimality of the proposed schemes.

\section{A. Outline}

The rest of the paper is organized as follows. Section II describes the system model, and Section III provides performance analysis. The proposed max-min SINR scheme is presented in Section IV and the convergence analysis is provided in Section V. The optimality of the proposed scheme is proved in Section VI. Section VII investigates a sub-optimal max-min SINR scheme. Complexity analysis and a proposed user assignment scheme are presented in Section VIII and Section IX, respectively. Finally, Section X provides numerical results while Section XI concludes the paper.

\section{B. Notation}

The following notations are adopted in the rest of the paper. Uppercase and lowercase boldface letters are used for matrices and vectors, respectively. The notation $\mathbb{E}\{\cdot\}$ denotes expectation. $|\cdot|$ stands for absolute value. The conjugate transpose of vector $\mathbf{x}$ is $\mathbf{x}^{H}$, and $\mathbf{X}^{T}$ denotes the transpose of matrix $\mathbf{X}$. In addition, $x \sim \mathcal{C N}\left(0, \sigma^{2}\right)$ represents a zeromean circularly symmetric complex Gaussian random variable with variance $\sigma^{2}$.

\section{SYSTEM MODEL}

We consider uplink transmission in a cell-free Massive MIMO system with $M$ single-antenna APs and $K$ randomly distributed single-antenna users in the area, as shown in Fig. 1. 
The channel coefficient between the $k$ th user and the $m$ th AP, $g_{m k}$, is modeled as [4]

$$
g_{m k}=\sqrt{\beta_{m k}} h_{m k},
$$

where $\beta_{m k}$ denotes the large-scale fading and $h_{m k} \sim$ $\mathcal{C N}(0,1)$ represents small-scale fading between the $k$ th user and the $m$ th AP.

\section{A. Uplink Channel Estimation}

In order to estimate channel coefficients in the uplink, the APs employ an minimum mean-square error (MMSE) estimator. During the training phase, all $K$ users simultaneously transmit their pilot sequences of length $\tau$ symbols to the APs. Let $\sqrt{\tau} \phi_{k} \in C^{\tau \times 1}$, where $\left\|\phi_{k}\right\|^{2}=1$, be the pilot sequence assigned to the $k$ th user. Then, the received signal at the $m$ th AP is given by

$$
\mathbf{y}_{m}^{p}=\sqrt{\tau p_{p}} \sum_{k=1}^{K} g_{m k} \boldsymbol{\phi}_{k}+\mathbf{w}_{m}^{p},
$$

where vector $\mathbf{w}_{m}^{p} \in \mathbb{C}^{\tau \times 1}$ is the noise whose elements are i.i.d $\mathcal{C N}(0,1)$. Next, the APs exploit the pilot sequence $\phi_{k}$ to correlate the received signal with the pilot sequence as follows [4]:

$$
\check{\mathbf{y}}_{m, k}^{p}=\boldsymbol{\phi}_{k}^{H} \mathbf{y}_{m}^{p}=\sqrt{\tau p_{p}} g_{m k}+\sqrt{\tau p_{p}} \sum_{k^{\prime} \neq k}^{K} g_{m k^{\prime}} \boldsymbol{\phi}_{k}^{H} \phi_{k^{\prime}}+\dot{w}_{m k}^{p},
$$

where $\dot{w}_{m k}^{p} \triangleq \boldsymbol{\phi}_{k}^{H} \mathbf{w}_{m}^{p}$. The linear MMSE estimate of $g_{m k}$ is

$$
\begin{aligned}
\hat{g}_{m k} & =\frac{\mathbb{E}\left\{g_{m k} \check{\mathbf{y}}_{m, k}^{p}\right\}}{\mathbb{E}\left\{\left|\check{\mathbf{y}}_{m, k}^{p}\right|^{2}\right\}} \check{\mathbf{y}}_{m, k}^{p} \\
& =c_{m k}\left(\sqrt{\tau p_{p}} g_{m k}+\sqrt{\tau p_{p}} \sum_{k^{\prime} \neq k}^{K} g_{m k^{\prime}} \phi_{k}^{H} \boldsymbol{\phi}_{k^{\prime}}+\dot{w}_{m k}^{p}\right),
\end{aligned}
$$

where $c_{m k}$ is obtained as [4]

$$
c_{m k}=\frac{\sqrt{\tau p_{p}} \beta_{m k}}{\tau p_{p} \sum_{k^{\prime}=1}^{K} \beta_{m k^{\prime}}\left|\phi_{k}^{H} \boldsymbol{\phi}_{k^{\prime}}\right|^{2}+1} .
$$

Note that, as in [4], we assume that the large-scale fading, $\beta_{m k}$, is known. The estimated channels in (3) are used by the APs to design the receiver filter coefficients and determine power allocations at users to maximize the minimum rate of the users. In this paper, we investigate the cases of both random pilot assignment and orthogonal pilots in cell-free Massive MIMO. Here the term "orthogonal pilots" refers to the case where unique orthogonal pilots are assigned to all users, while in "random pilot assignment" each user is randomly assigned a pilot sequence from a set of orthogonal sequences of length $\tau(<K)$, following the approach of [4] and [26].

\section{B. Uplink Transmission}

In this subsection, we consider the uplink data transmission, where all users send their signals to the APs. The transmitted signal from the $k$ th user is represented by

$$
x_{k}=\sqrt{\rho q_{k}} s_{k},
$$

where $s_{k}\left(\mathbb{E}\left\{\left|s_{k}\right|^{2}\right\}=1\right)$ and $q_{k}$ denote the transmitted symbol and the transmit power from the $k$ th user, respectively. Moreover, $\rho$ refers to the normalized uplink SNR. The received signal at the $m$ th AP from all users is given by

$$
y_{m}=\sqrt{\rho} \sum_{k=1}^{K} g_{m k} \sqrt{q_{k}} s_{k}+n_{m},
$$

where $n_{m} \sim \mathcal{C N}(0,1)$ is the noise at the $m$ th AP. In addition, a matched filtering approach is employed at the APs, in that the received signal is weighted appropriately. More precisely, the received signal at the $m$ th AP, $y_{m}$, is first multiplied by $\hat{g}_{m k}^{*}$. The resulting $\hat{g}_{m k}^{*} y_{m}$ is then forwarded to the CPU for signal detection. In order to improve achievable rate, the forwarded signal is further multiplied by a receiver filter coefficient at the CPU. The aggregated received signal at the CPU can be written as

$$
\begin{aligned}
r_{k} & =\sum_{m=1}^{M} u_{m k} \hat{g}_{m k}^{*} y_{m} \\
& =\sqrt{\rho} \sum_{k^{\prime}=1}^{K} \sum_{m=1}^{M} u_{m k} \hat{g}_{m k}^{*} g_{m k^{\prime}} \sqrt{q_{k^{\prime}}} s_{k^{\prime}}+\sum_{m=1}^{M} u_{m k} \hat{g}_{m k}^{*} n_{m} .
\end{aligned}
$$

By collecting all the coefficients $u_{m k}, \forall m$ corresponding to the $k$ th user, we define $\mathbf{u}_{k}=\left[u_{1 k}, u_{2 k}, \cdots, u_{M k}\right]^{T}$ and without loss of generality, it is assumed that $\left\|\mathbf{u}_{k}\right\|=1$. The optimal solution for $\mathbf{u}_{k}, q_{k}, \forall k$ for the considered maxmin SINR approach is investigated in Section IV. Similar to [4], [6], and [13], we assume that the APs are connected to the CPU via perfect backhaul connections. Such perfect backhaul links might be established through fiber links between the APs and the CPU. Moreover, based on [27], copperbased backhaul links can provide a capacity of $750 \mathrm{Mbits} / \mathrm{s}$ for a maximum distance of $1.5 \mathrm{~km}$ between the APs and the CPU. In [28]-[30], the authors show that exploiting optimal uniform quantization and wireless microwave links with capacity $100 \mathrm{Mbits} / \mathrm{s}$ [31], the performance of limitedbackhaul cell-free Massive MIMO system closely approaches the performance of cell-free Massive MIMO with perfect backhaul links.

\section{iII. Performance Analysis}

In this section, we derive the achievable rate for the considered system model by following a similar approach to [4]. Note that the main difference between the proposed approach and the scheme in [4] is the new set of receiver filter coefficients which are introduced at the CPU to improve the achievable user rate. The benefits of the proposed approach in terms of the achievable uplink rate are demonstrated by the numerical results in Section V. In deriving the achievable rate of each user, it is assumed that the CPU exploits only the knowledge 
of channel statistics between the users and APs in detecting data from the received signal in (7). Without loss of generality, the aggregate received signal in (7) can be written as

$$
\begin{aligned}
r_{k} & \underbrace{\sqrt{\rho \mathbb{E}\left\{\sum_{m=1}^{M} u_{m k} \hat{g}_{m k}^{*} g_{m k} \sqrt{q_{k}}\right\}} s_{k}}_{\mathrm{DS}_{k}} \\
& +\underbrace{\sqrt{\rho}\left(\sum_{m=1}^{M} u_{m k} \hat{g}_{m k}^{*} g_{m k} \sqrt{q_{k}}-\mathbb{E}\left\{\sum_{m=1}^{M} u_{m k} \hat{g}_{m k}^{*} g_{m k} \sqrt{q_{k}}\right\}\right)}_{\mathrm{BU}_{k}} s_{k} \\
& +\sum_{k^{\prime} \neq k}^{\sum_{\mathrm{IUI}_{k k^{\prime}}}^{K} \sqrt{\rho} \sum_{m=1}^{M} u_{m k} \hat{g}_{m k}^{*} g_{m k^{\prime}} \sqrt{q_{k^{\prime}}}} s_{k^{\prime}}+\underbrace{\sum_{m=1}^{M} u_{m k} \hat{g}_{m k}^{*} n_{m}}_{\mathrm{TN}_{k}}
\end{aligned}
$$

where $\mathrm{DS}_{k}$ and $\mathrm{BU}_{k}$ denote the desired signal (DS) and beamforming uncertainty (BU) for the $k$ th user, respectively, and $\mathrm{IUI}_{k k^{\prime}}$ represents the inter-user-interference (IUI) caused by the $k^{\prime}$ th user. In addition, $\mathrm{TN}_{k}$ accounts for the total noise (TN) following the matched filtering. The corresponding SINR of the received signal in (8) can be defined by considering the worst-case of the uncorrelated Gaussian noise as follows [4]:

$$
\operatorname{SINR}_{k}^{\mathrm{UP}}=\frac{\left|\mathrm{DS}_{k}\right|^{2}}{\mathbb{E}\left\{\left|\mathrm{BU}_{k}\right|^{2}\right\}+\sum_{k^{\prime} \neq k}^{K} \mathbb{E}\left\{\left|\mathrm{IUI}_{k k^{\prime}}\right|^{2}\right\}+\mathbb{E}\left\{\left|\mathrm{TN}_{k}\right|^{2}\right\}}
$$

Based on the SINR definition in (9), the achievable uplink rate of the $k$ th user is defined in the following theorem:

Theorem 1: By employing the matched filtering approach at the APs, the achievable uplink rate of the kth user in the cell-free Massive MIMO system with $K$ randomly distributed single-antenna users and $M$ single-antenna APs is given by (10), shown at the bottom of this page. Note that in (10), we have

$$
\begin{aligned}
\boldsymbol{\Gamma}_{k} & =\left[\gamma_{1 k}, \gamma_{2 k}, \cdots, \gamma_{M k}\right]^{T}, \\
\boldsymbol{u}_{k} & =\left[u_{1 k}, u_{2 k}, \cdots, u_{M k}\right]^{T}, \\
\boldsymbol{\Delta}_{k k^{\prime}} & =\left[\frac{\gamma_{1 k} \beta_{1 k^{\prime}}}{\beta_{1 k}}, \frac{\gamma_{2 k} \beta_{2 k^{\prime}}}{\beta_{2 k}}, \cdots, \frac{\gamma_{M k} \beta_{M k^{\prime}}}{\beta_{M k}}\right]^{T}, \\
\boldsymbol{R}_{k} & =\operatorname{diag}\left[\gamma_{1 k}, \gamma_{2 k}, \cdots, \gamma_{M k}\right], \\
\boldsymbol{D}_{k k^{\prime}} & =\operatorname{diag}\left[\beta_{1 k^{\prime}} \gamma_{1 k}, \beta_{2 k^{\prime}} \gamma_{2 k}, \cdots, \beta_{M k^{\prime}} \gamma_{M k}\right] .
\end{aligned}
$$

Proof: Please refer to Appendix A.

Note that the achievable rate in (10) is a function of only large-scale fading which changes less often than the actual channel. Hence, the rate formula and accordingly the power coefficients only need to be calculated when the large-scale fading changes. Therefore, the APs do not need frequently to update the CPU with the instantaneous channel state and the user rates will change only when the positions of the users change. Moreover, in cell-free Massive MIMO, due to the channel hardening property, detection using only the channel statistics is nearly optimal [4].

\section{Proposed MaX-Min SINR Scheme}

In this section, we formulate the max-min user-fairness problem in the cell-free Massive MIMO, where the minimum uplink rates of all users is maximized while satisfying the per-user power constraint. This max-min rate problem can be formulated as the following optimization framework:

$$
\begin{gathered}
P_{1}: \max _{q_{k}, \mathbf{u}_{k}} \min _{k=1, \cdots, K} R_{k}^{\mathrm{UP},} \\
\text { s.t. }\left\|\mathbf{u}_{k}\right\|=1, \quad \forall k, \\
\quad 0 \leq q_{k} \leq p_{\max }^{(k)}, \quad \forall k,
\end{gathered}
$$

where $p_{\max }^{(k)}$ is the maximum transmit power available at user $k$. From (10), it can be observed that in the denominator of the expression for the uplink SINR, the power coefficients $q_{k^{\prime}}, k^{\prime} \neq k$ are coupled with the receiver filter $\mathbf{u}_{k}$. Therefore, it is not possible to define a new variable $\mathbf{w}_{k}=$ $\sqrt{q_{k}} \mathbf{u}_{k}$, and solve the problem jointly in terms of $\mathbf{u}_{k}$ and $q_{k}$. As a result, Problem $P_{1}$ is not jointly convex in terms of $\mathbf{u}_{k}$ and power allocation $q_{k}, \forall k$. Therefore, this problem cannot be directly solved through existing convex optimization software. To tackle this non-convexity issue, we decouple the original problem $P_{1}$ into two sub-problems: receiver filter coefficient design (i.e., $\mathbf{u}_{k}$ ) and the power allocation problem. To obtain a solution for Problem $P_{1}$, these sub-problems are alternately solved as explained in the following subsections.

\section{A. Receiver Filter Coefficient Design}

In this subsection, we solve the receiver coefficient design problem to maximize the uplink rate of each user for a given set of transmit power allocations at all users. By following the analysis in [17], [22], and [23], the receiver filter coefficients (i.e., $\mathbf{u}_{k}, \forall k$ ) can be obtained by independently maximizing the uplink SINR of each user. Therefore, the optimal receiver filter coefficients for all users for a given set of transmit power allocations can be determined by solving the following optimization problem (13a) and (13b), shown at the bottom of this page.

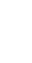
.

$$
\begin{aligned}
R_{k}^{\mathrm{UP}}=\log _{2}\left(1+\frac{\mathbf{u}_{k}^{H}\left(q_{k} \boldsymbol{\Gamma}_{k} \boldsymbol{\Gamma}_{k}^{H}\right) \mathbf{u}_{k}}{\mathbf{u}_{k}^{H}\left(\sum_{k^{\prime} \neq k}^{K} q_{k^{\prime}}\left|\boldsymbol{\phi}_{k}^{H} \boldsymbol{\phi}_{k^{\prime}}\right|^{2} \boldsymbol{\Delta}_{k k^{\prime}} \boldsymbol{\Delta}_{k k^{\prime}}^{H}+\sum_{k^{\prime}=1}^{K} q_{k^{\prime}} \mathbf{D}_{k k^{\prime}}+\frac{1}{\rho} \mathbf{R}_{k}\right) \mathbf{u}_{k}}\right) . \\
P_{2}: \max _{\mathbf{u}_{k}} \frac{\mathbf{u}_{k}^{H}\left(q_{k} \boldsymbol{\Gamma}_{k} \boldsymbol{\Gamma}_{k}^{H}\right) \mathbf{u}_{k}}{\mathbf{u}_{k}^{H}\left(\sum_{k^{\prime} \neq k}^{K} q_{k^{\prime}}\left|\boldsymbol{\phi}_{k}^{H} \boldsymbol{\phi}_{k^{\prime}}\right|^{2} \boldsymbol{\Delta}_{k k^{\prime}} \boldsymbol{\Delta}_{k k^{\prime}}^{H}+\sum_{k^{\prime}=1}^{K} q_{k^{\prime}} \mathbf{D}_{k k^{\prime}}+\frac{1}{\rho} \mathbf{R}_{k}\right) \mathbf{u}_{k}} \\
\text { s.t. }\left\|\mathbf{u}_{k}\right\|=1, \quad \forall k .
\end{aligned}
$$




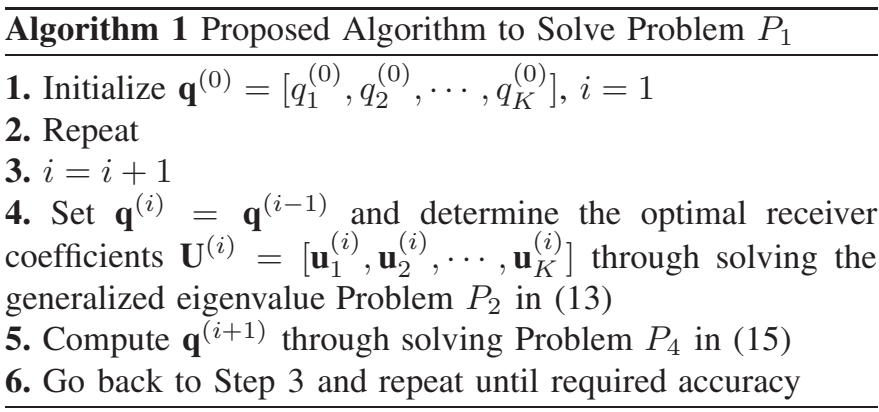

Problem $P_{2}$ is a generalized eigenvalue problem [19], where the optimal solutions can be obtained by determining the generalized eigenvalue of the matrix pair $\mathbf{A}_{k}=q_{k} \boldsymbol{\Gamma}_{k} \boldsymbol{\Gamma}_{k}^{H}$ and $\mathbf{B}_{k}=\sum_{k^{\prime} \neq k}^{K} q_{k^{\prime}}\left|\boldsymbol{\phi}_{k}^{H} \boldsymbol{\phi}_{k^{\prime}}\right|^{2} \boldsymbol{\Delta}_{k k^{\prime}} \boldsymbol{\Delta}_{k k^{\prime}}^{H}+\sum_{k^{\prime}=1}^{K} q_{k^{\prime}} \mathbf{D}_{k k^{\prime}}+\frac{1}{\rho} \mathbf{R}_{k}$ corresponding to the maximum generalized eigenvalue.

\section{B. Power Allocation}

In this subsection, we solve the power allocation problem for a given set of fixed receiver filter coefficients which can be formulated as the following max-min problem:

$$
\begin{gathered}
P_{3}: \max _{q_{k}} \min _{k=1, \cdots, K} \operatorname{SINR}_{k}^{\mathrm{UP}}, \\
\text { s.t. } 0 \leq q_{k} \leq p_{\max }^{(k)} .
\end{gathered}
$$

Without loss of generality, Problem $P_{3}$ can be rewritten by introducing a new slack variable as

$$
\begin{aligned}
P_{4}: \quad \max _{t, q_{k}} t, & \\
\text { s.t. } & 0 \leq q_{k} \leq p_{\max }^{(k)}, \quad \forall k, \\
& \operatorname{SINR}_{k}^{\mathrm{UP}} \geq t, \quad \forall k .
\end{aligned}
$$

Proposition 1: Problem $P_{4}$ can be formulated into a standard GP.

Proof: Please refer to Appendix B.

Therefore, this problem can be efficiently solved through existing convex optimization software. Based on these two sub-problems, an iterative algorithm is developed by alternately solving each sub-problem at each iteration. The proposed algorithm is summarized in Algorithm 1.

\section{Convergence Analysis}

In this section, the convergence analysis of the proposed Algorithm 1 is provided. Two sub-problems are alternately solved to determine the solution to Problem $P_{1}$. At each iteration, one of the design parameters is determined by solving the corresponding sub-problem while other design variable is fixed. Note that each sub-problem provides an optimal solution for the other given design variable. At the $i$ th iteration, the receiver filter coefficients $\mathbf{u}_{k}^{(i)}, \forall k$ are determined for a given power allocation $\mathbf{q}^{(i)}$ and similarly, the power allocation $\mathbf{q}^{(i+1)}$ is updated for a given set of receiver filter coefficients $\mathbf{u}_{k}^{(i)}, \forall k$. The optimal power allocation $\mathbf{q}^{(i+1)}$ obtained for a given $\mathbf{u}_{k}^{(i)}$ achieves an uplink rate greater than or equal to that of the previous iteration. In addition, the power allocation $\mathbf{q}^{(i)}$ is also a feasible solution in determining $\mathbf{q}^{(i+1)}$ as the receiver filter coefficients $\mathbf{u}_{k}^{(i+1)}, \forall k$ are determined for a given $\mathbf{q}^{(i)}$. This reveals that the achieved uplink rate monotonically increases with each iteration, which can be also observed from the simulation results presented in Figs. 8 and 9. As the achievable uplink max-min rate is upper bounded by a certain value for a given set of per-user power constraints, the proposed algorithm converges to a particular solution. Fortunately, the proposed Algorithm 1 converges to the optimal solution, as we will prove by establishing the uplink-downlink duality in the following section.

\section{Optimality of the Proposed MAX-MIN SINR ALGORITHM}

In this section, we prove the optimality of the proposed maxmin SINR scheme in Algorithm 1. In general, converting the original non-convex problem into two sub-problems would remove the global optimality. However, the global optimality of the proposed Algorithm 1 can be proved as follows: first, we show that the solution of the original max-min Problem $P_{1}$ can be obtained by solving an uplink problem with an equivalent total power constraint instead of the peruser power constraint. Then, an uplink-downlink duality is established by proving that the same SINRs can be achieved in both the uplink and the downlink with an equivalent total power constraint. In other words, the same SINRs in the uplink Problem $P_{1}$ can be realized by solving an equivalent downlink problem. Finally, we present a bisection approach to determine the optimal solution of the equivalent downlink problem. Since both the uplink Problem $P_{1}$ and the equivalent downlink problem achieve the same SINRs and the solution of the downlink problem is optimal, it is straightforward to conclude that Algorithm 1 yields the optimal solution for the considered uplink max-min SINR problem in $P_{1}$. The details of the proof are provided in the following subsections.

\section{A. Equivalent Max-Min Uplink Problem}

In this subsection, we show that both Problem $P_{1}$ with peruser power constraint and the uplink max-min fairness problem with the total power constraint achieve the same user rate. In the total power constraint, the maximum available transmit power is defined as the summation of all users' transmit power from the solution of Problem $P_{1}$, which can be written as follows:

$$
P_{\text {tot }}^{c}=\sum_{k=1}^{K} q_{k}^{*},
$$

where $q_{k}^{*}$ is the power allocated to the $k$ th user obtained by solving problem $P_{1}$ (Algorithm 1). The equivalent uplink maxmin problem with this total power constraint can be formulated as follows:

$$
\begin{gathered}
P_{5}: \max _{q_{k}, \mathbf{u}_{k}} \min _{k=1, \cdots, K} R_{k}^{\mathrm{UP}}, \\
\text { s.t. }\left\|\mathbf{u}_{k}\right\|=1, \quad \forall k, \\
\sum_{k=1}^{K} q_{k} \leq P_{\text {tot }}^{c} .
\end{gathered}
$$

Similar to the original Problem $P_{1}$, Problem $P_{5}$ is not jointly convex in terms of receiver filter coefficients $\mathbf{u}_{k}$ and power allocation $q_{k}, \forall k$. However, we modify Algorithm 1 to incorporate the total power constraint in Problem $P_{5}$. Similar to the 
alternate optimization approach for Problem $P_{1}$, Problem $P_{5}$ is decoupled into receiver filter coefficient design and power allocation sub problems. The same generalized eigenvalue problem in Problem $P_{2}$ is solved to determine the receiver filter coefficients whereas the GP formulation in $P_{4}$ is adapted to incorporate the total power constraint (17c). This is a convex constraint (posynomial function in terms of power allocation) and the power allocation problem (GP) with the equivalent total power constraint remains as a convex problem.

Lemma 1: Both the original Problem $P_{1}$ and Problem $P_{5}$ yield the same solution with per-user power constraint and equivalent total power constraint.

Proof: Please refer to Appendix C.

\section{B. Uplink-Downlink Duality for Cell-Free Massive MIMO}

In this subsection, we establish an uplink-downlink duality for cell-free Massive MIMO systems. In particular, it is shown that the same SINRs (or rate regions) can be realized for all users in the uplink and the downlink with the equivalent total power constraints, respectively [22]-[24], [32]. In other words, the same set of filter coefficients can be utilized in the uplink and the downlink to achieve the same SINRs for all users with different user power allocations. The following theorem defines the achievable downlink rate for cell-free Massive MIMO systems:

Theorem 2: By employing conjugate beamforming at the APs, the achievable downlink rate of the kth user in the cell-free Massive MIMO system with $K$ randomly distributed single-antenna users and $M$ single-antenna APs is given by (18), shown at the bottom of this page.

Proof: This can be derived by following the same approach as for the uplink in Theorem 1.

Note that the symbol $\boldsymbol{\Lambda}_{k^{\prime} k}$, in (18), is defined as $\boldsymbol{\Lambda}_{k^{\prime} k}=\left[\frac{\gamma_{1 k^{\prime}} \beta_{1 k}}{\beta_{1 k^{\prime}}}, \frac{\gamma_{2 k^{\prime}} \beta_{2 k}}{\beta_{2 k^{\prime}}}, \cdots, \frac{\gamma_{M k^{\prime}} \beta_{M k}}{\beta_{M k^{\prime}}}\right]^{T}$, and $\boldsymbol{\Upsilon}_{k^{\prime} k}$ denotes the diagonal matrix whose diagonal entries are $\left[\gamma_{1 k^{\prime}} \beta_{1 k}, \gamma_{2 k^{\prime}} \beta_{2 k}, \cdots, \gamma_{M k^{\prime}} \beta_{M k}\right]$. In addition, $p_{k}, \forall k$ denotes the downlink power allocation for the $k$ th user. Moreover, the uplink SINR is given in (19), shown at the bottom of this page. The following Theorem provides the required condition to establish the uplink-downlink duality for cell-free Massive MIMO systems:

Theorem 3: By employing matched filtering in the uplink and conjugate beamforming in the downlink, to realize the same SINR tuples in both the uplink and the downlink of a cell-free Massive MIMO system, with the same filter coefficients and different transmit power allocations, the following condition should be satisfied:

$$
\sum_{m=1}^{M} \sum_{k=1}^{K} \gamma_{m k}\left|w_{m k}\right|^{2}=\sum_{k=1}^{K} q_{k}^{*}=P_{t o t}^{c},
$$

where $w_{m k}$ denotes the $(m, k)$-th entry of matrix $\boldsymbol{W}$ which is defined as follows:

$$
\boldsymbol{W}=\left[\sqrt{p_{1}} \boldsymbol{u}_{1}, \sqrt{p_{2}} \boldsymbol{u}_{2}, \cdots, \sqrt{p_{K}} \boldsymbol{u}_{K}\right] .
$$

Proof: Please refer to Appendix D.

\section{Equivalent Max-Min Downlink Problem}

In this subsection, we present an optimal approach to solve the max-min SINR downlink problem with the equivalent total power constraint. This user-fairness problem can be formulated as follows:

$$
\begin{gathered}
P_{6}: \max _{p_{k}, \mathbf{u}_{k}} \min _{k=1, \cdots, K} R_{k}^{\mathrm{DL}}, \\
\text { s.t. }\left\|\mathbf{u}_{k}\right\|=1, \quad \forall k, \\
\sum_{k=1}^{K} p_{k} \leq P_{\text {tot }}^{c},
\end{gathered}
$$

where $R_{k}^{\mathrm{DL}}=\log _{2}\left(1+\operatorname{SINR}_{k}^{\mathrm{DL}}\right)$, and $\operatorname{SINR}_{k}^{\mathrm{DL}}$ is defined in (18). This problem is difficult to jointly solve in terms of transmit filter coefficients $\mathbf{u}_{k}$ 's and power allocations $p_{k}$ 's. However, similar to [4], it can be reformulated by introducing a new variable by coupling both of these variables as follows:

$$
\begin{aligned}
P_{7}: & \max _{\mathbf{W}} \min _{k=1, \cdots, K} R_{k}^{\mathrm{DL}}, \\
& \text { s.t. } \sum_{m=1}^{M} \sum_{k=1}^{K} \gamma_{m k}\left|w_{m k}\right|^{2} \leq P_{\text {tot }}^{c} .
\end{aligned}
$$

$$
\begin{aligned}
& \operatorname{SINR}_{k}^{\mathrm{DL}}(\mathbf{U}, \mathbf{p})=\frac{\mathbf{u}_{k}^{H}\left(p_{k} \boldsymbol{\Gamma}_{k} \boldsymbol{\Gamma}_{k}^{H}\right) \mathbf{u}_{k}}{\sum_{k^{\prime} \neq k}^{K} \mathbf{u}_{k^{\prime}}^{H} p_{k^{\prime}}\left|\boldsymbol{\phi}_{k^{\prime}}^{H} \boldsymbol{\phi}_{k}\right|^{2} \boldsymbol{\Lambda}_{k^{\prime} k} \boldsymbol{\Lambda}_{k^{\prime} k}^{H} \mathbf{u}_{k^{\prime}}+\sum_{k^{\prime}=1}^{K} \mathbf{u}_{k^{\prime}}^{H} p_{k^{\prime}} \mathbf{\Upsilon}_{k^{\prime} k} \mathbf{u}_{k^{\prime}}+\frac{1}{\rho}} . \\
& \operatorname{SINR}_{k}^{\mathrm{UP}}(\mathbf{U}, \mathbf{q})=\frac{\mathbf{u}_{k}^{H}\left(q_{k} \boldsymbol{\Gamma}_{k} \boldsymbol{\Gamma}_{k}^{H}\right) \mathbf{u}_{k}}{\mathbf{u}_{k}^{H}\left(\sum_{k^{\prime} \neq k}^{K} q_{k^{\prime}}\left|\boldsymbol{\phi}_{k}^{H} \boldsymbol{\phi}_{k^{\prime}}\right|^{2} \boldsymbol{\Delta}_{k k^{\prime}} \boldsymbol{\Delta}_{k k^{\prime}}^{H}+\sum_{k^{\prime}=1}^{K} q_{k^{\prime}} \mathbf{D}_{k k^{\prime}}+\frac{1}{\rho} \mathbf{R}_{m k}\right) \mathbf{u}_{k}} . \\
& P_{8}: \min _{\mathbf{W}} \sum_{m=1}^{M} \sum_{k=1}^{K} \gamma_{m k}\left|w_{m k}\right|^{2} \\
& \text { s.t. } \frac{\mathbf{w}_{k}^{H}\left(\boldsymbol{\Gamma}_{k} \boldsymbol{\Gamma}_{k}^{H}\right) \mathbf{w}_{k}}{\sum_{k^{\prime} \neq k}^{K} \mathbf{w}_{k^{\prime}}^{H}\left|\boldsymbol{\phi}_{k^{\prime}}^{H} \boldsymbol{\phi}_{k}\right|^{2} \boldsymbol{\Lambda}_{k^{\prime} k} \boldsymbol{\Lambda}_{k^{\prime} k}^{H} \mathbf{w}_{k^{\prime}}+\sum_{k^{\prime}=1}^{K} \mathbf{w}_{k^{\prime}}^{H} \mathbf{\Upsilon}_{k^{\prime} k} \mathbf{w}_{k^{\prime}}+\frac{1}{\rho}} \geq t, \\
& \sum_{m=1}^{M} \sum_{k=1}^{K} \gamma_{m k}\left|w_{m k}\right|^{2} \leq P_{\text {tot }}^{c} \text {, }
\end{aligned}
$$


It can be easily shown that Problem $P_{7}$ is quasi-convex, therefore a bisection approach can be exploited to obtain the optimal solution for the original Problem $P_{7}$ by sequentially solving the following power minimization problem for a given target SINR $t$ at all users (24a)-(24c), shown at the bottom of this page, where $\mathbf{w}_{k}$ represents the $k$ th column of the matrix $\mathbf{W}$ defined in (21). Second order cone programming (SOCP) can be exploited to reformulate Problem $P_{8}$ as a convex one. More precisely, for a given $t$, Problem $P_{8}$ can be reformulated as follows:

$$
\begin{aligned}
P_{8}^{\text {rewrite }}: & \min _{\mathbf{W}} \sum_{m=1}^{M} \sum_{k=1}^{K} \gamma_{m k}\left|w_{m k}\right|^{2}, \\
\text { s.t. } & \left\|\mathbf{z}_{k}\right\| \leq \frac{\sum_{m=1}^{M}\left[\boldsymbol{\Gamma}_{k}\right]_{m} w_{m k}}{\sqrt{t}}, \forall k, \\
& \sum_{m=1}^{M}\left[\boldsymbol{\Lambda}_{k^{\prime} k}\right]_{m} w_{m k^{\prime}} \leq \chi_{k^{\prime} k}, \forall k^{\prime} \neq k, \\
& \sum_{m=1}^{M}\left[\mathbf{\Upsilon}_{k^{\prime} k}\right]_{m} w_{m k^{\prime}}^{2} \leq \psi_{k^{\prime} k}^{2}, \forall k, \\
& \sum_{m=1}^{M} \sum_{k=1}^{K} \gamma_{m k}\left|w_{m k}\right|^{2} \leq P_{\mathrm{tot}}^{c},
\end{aligned}
$$

where $\chi_{k^{\prime} k}$ and $\psi_{k^{\prime} k}^{2}$ are slack variables, and $[\mathbf{x}]_{n}$ represents the $n$th element of vector $\mathbf{x}$. Moreover, we have

$$
\begin{array}{r}
\mathbf{z}_{k} \triangleq\left[\chi_{1 k} \boldsymbol{\phi}_{1}^{H} \boldsymbol{\phi}_{k}, \cdots, \chi_{(k-1) k} \boldsymbol{\phi}_{k-1}^{H} \boldsymbol{\phi}_{k}, \chi_{(k+1) k} \boldsymbol{\phi}_{k+1}^{H} \boldsymbol{\phi}_{k}, \cdots,\right. \\
\left.\chi_{K k} \boldsymbol{\phi}_{K}^{H} \boldsymbol{\phi}_{k}, \psi_{1 k}, \cdots, \psi_{K k}, \frac{1}{\sqrt{\rho}}\right] . \quad \text { (26) }
\end{array}
$$

It can be seen that (25b) represents second order cone (SOC) [33]. Hence, Problem $P_{8}^{\text {rewrite }}$ is a SOCP.

Therefore, the optimal solution for Problem $P_{6}$ can be derived by extracting the normalized transmit filter coefficients $\mathbf{u}_{k}$ 's and power allocations $p_{k}$ 's as

$$
\begin{aligned}
& p_{k}^{*}=\left\|\mathbf{w}_{k}^{*}\right\|^{2}, \quad \forall k, \\
& \mathbf{u}_{k}^{*}=\frac{\mathbf{w}_{k}^{*}}{\left\|\mathbf{w}_{k}^{*}\right\|}, \forall k,
\end{aligned}
$$

where $\mathbf{w}_{k}^{*}$ 's are the optimal solution of Problem $P_{7}$. Note that constraint (23b) is an equivalent total power constraint to the per-user power constraint in the original uplink maxmin SINR problem in $P_{1}$, which is a more relaxed constraint than the per-user power constraint in $P_{1}$. However, it is already shown in the previous sub-section that the same SINRs can be realized in both the uplink and the downlink with peruser and the equivalent total power constraints. In addition, the SINRs achieved in the downlink problem in $P_{7}$ are optimal and therefore the SINRs achieved in Problem $P_{1}$ is optimal. Next, let us again consider the uplink max-min SINR Problems $P_{1}$ and $P_{5}$. After solving the uplink maxmin SINR with total power (with the maximum available power $P_{\text {tot }}^{c}=\sum_{k=1}^{K} q_{k}^{*}$ defined in Problem $P_{5}$ ), and solving the uplink max-min SINR with per-user power constraints (Problem $P_{1}$ ), we observe that the obtained power allocation for all users $\left(q_{k}, \forall k\right)$ after solving Problem $P_{1}$ and Problem $P_{5}$ are exactly the same. Moreover, after solving Problem $P_{5}$ using the proposed Algorithm 1, it is observed that at least one of the users always consumes the maximum power (i.e., there always exists one user with $\left.q_{k}^{*}=p_{\max }^{(k)}\right)$. However, it is easy to prove that it is not possible to improve the max-min rate of the system by increasing the power of other users since in this case we would have to decrease the power of user with $q_{k}^{*}=p_{\max }^{(k)}$, which decreases the rate of this user, and hence the max-min rate. This validates the optimality of the proposed max-min SINR scheme in Algorithm 1.

\section{Sub-Optimal UPLink MAX-Min SINR}

In this section, we revisit the bisection search based uplink max-min SINR scheme presented in [4]. First, this bisection scheme is summarized and then, we propose another approach to solve this max-min SINR problem by formulating it into a convex optimization framework. This scheme is developed by appropriately allocating transmit powers at each user with an matched filtering technique at the APs. However, no receiver filter coefficient design has been considered at the CPU to enhance the uplink rate as in the previous section. The achievable rate of the $k$ th user is derived in (28), shown at the bottom of this page, where $\eta_{k}$ is the allocated transmit power at user $k$ [4]. For this scenario, the uplink max-min SINR problem can be formulated as the following max-min problem:

$$
\begin{aligned}
P_{9}: & \max _{\eta_{k} \geq 0} \min _{k} R_{k}^{\mathrm{UP}}, \\
& \text { s.t. } 0 \leq \eta_{k} \leq p_{\max }^{(k)} .
\end{aligned}
$$

\section{A. Bisection Search Method}

In this subsection, we present the bisection search method for this quasi-linear problem. As this problem cannot be directly solved in this present form, a series of power minimization problems is solved by setting the same target rate for all users and the corresponding target rate is modified in the next iteration according to the feasibility or infeasibility of the power minimization problem at each iteration. The feasibility of the following power minimization problem is verified for a given target SINR $t$ at all users in each iteration of the bisection search [4]:

$$
\begin{aligned}
P_{10}: \min _{\eta_{k}} & \sum_{k=1}^{K} \eta_{k}, \\
\text { s.t. } 0 \leq \eta_{k} \leq p_{\text {max }}^{(k)}, \quad \forall k, & \\
& \rho \sum_{k^{\prime} \neq k}^{K} \eta_{k^{\prime}}\left(\sum_{m=1}^{M} \gamma_{m k} \frac{\beta_{m k^{\prime}}}{\beta_{m k}}\right)^{2}\left|\boldsymbol{\phi}_{k}^{H} \boldsymbol{\phi}_{k^{\prime}}\right|^{2} t
\end{aligned}
$$

$$
R_{k}^{\mathrm{UP}}=\left(1+\frac{\rho \eta_{k}\left(\sum_{m=1}^{M} \gamma_{m k}\right)^{2}}{\rho \sum_{k^{\prime} \neq k}^{K} \eta_{k^{\prime}}\left(\sum_{m=1}^{M} \gamma_{m k} \frac{\beta_{m k^{\prime}}}{\beta_{m k}}\right)^{2}\left|\boldsymbol{\phi}_{k}^{H} \boldsymbol{\phi}_{k^{\prime}}\right|^{2}+\rho \sum_{k^{\prime}=1}^{K} \eta_{k^{\prime}} \sum_{m=1}^{M} \gamma_{m k} \beta_{m k^{\prime}}+\sum_{m=1}^{M} \gamma_{m k}}\right)
$$




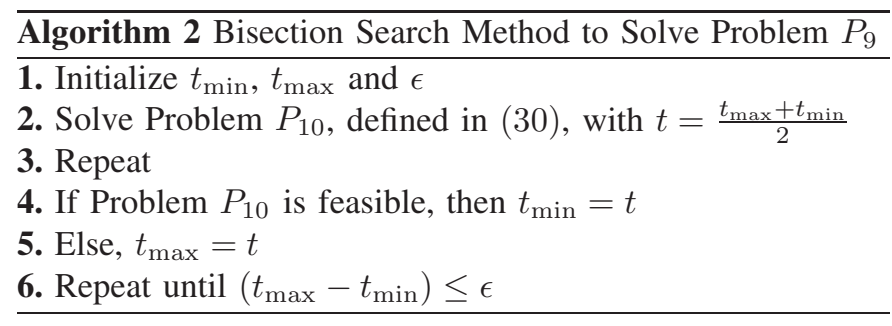

$$
\begin{aligned}
& +\rho \sum_{k^{\prime}=1}^{K} \eta_{k^{\prime}} \sum_{m=1}^{M} \gamma_{m k} \beta_{m k^{\prime}} t+\sum_{m=1}^{M} \gamma_{m k} t \\
& \leq \rho \eta_{k}\left(\sum_{m=1}^{M} \gamma_{m k}\right)^{2}, \forall k .
\end{aligned}
$$

In this bisection search approach, first an upper and lower bounds of the achievable SINR are set to $t_{\max }$ and $t_{\min }$, respectively and the initial target SINR $t$ is chosen as $\left(t_{\max }+\right.$ $\left.t_{\text {min }}\right) / 2$. If Problem $P_{10}$ is feasible for a given target SINR $t$, then the lower bound $t_{\min }$ will be set to $t$ and a new target SINR is chosen as $\left(t_{\max }+t_{\min }\right) / 2$ for the next iteration. This procedure is continued until the difference between the upper and the lower bounds is smaller than a predefined threshold $\epsilon$. This bisection search method based uplink max-min SINR scheme is summarized in Algorithm 2. Note that based on the analysis in [17], the bisection search method provides the optimal solution. In the rest of this section, we show that Problem $P_{9}$ can be reformulated as a standard GP, which does not require an iterative bisection search to find the optimal solution.

\section{B. Proposed Sub-Optimal Scheme}

In this subsection, we exploit GP (convex problem) to develop an efficient solution for Problem $P_{9}$ defined in (29). As mentioned in previous subsection, Problem $P_{9}$ cannot be directly solved through the optimization software. Consider the following optimization problem:

$$
\begin{aligned}
P_{11}: & \max _{t, \eta_{k}} t, \\
& \text { s.t. } 0 \leq \eta_{k} \leq p_{\max }^{(k)}, \quad \forall k, \\
& \operatorname{SINR}_{k}^{\mathrm{UP}} \geq t, \quad \forall k .
\end{aligned}
$$

Proposition 2: Problem $P_{11}$ can be reformulated into a GP.

Proof: The standard form of GP is defined in Appendix B. The SINR constraint in (31c) can be reformulated into the posynomial function. Following a simple transformation, the SINR constraint in (31c) can be represented by the following inequality:

$$
\eta_{k}^{-1}\left(\sum_{k^{\prime} \neq k}^{K} e_{k k^{\prime}} \eta_{k^{\prime}}+\sum_{k^{\prime}=1}^{K} f_{k k^{\prime}} \eta_{k^{\prime}}+r_{k}\right)<\frac{1}{t},
$$

where

$$
e_{k k^{\prime}}=\frac{\left(\sum_{m=1}^{M} \gamma_{m k} \frac{\beta_{m k^{\prime}}}{\beta_{m k}}\right)^{2}\left|\boldsymbol{\phi}_{k}^{H} \phi_{k^{\prime}}\right|^{2}}{\left(\sum_{m=1}^{M} \gamma_{m k}\right)^{2}},
$$

TABLE I

Computational Complexity of Different Problems

\begin{tabular}{cc}
\hline Problems & Required arithmetic operations \\
\hline Problem $P_{2}$, given by (13) & $O\left(K M^{3}\right)$ \\
\hline Problem $P_{4}$, given by (15) & $O\left(K^{\frac{7}{2}}\right)$ \\
\hline Problem $P_{10}$, given by (30) & $\log _{2}\left(\frac{t_{\max }-t_{\min }}{\epsilon}\right) O\left(K^{4}\right)$ \\
\hline Problem $P_{11}$, given by (31) & $O\left(K^{\frac{7}{2}}\right)$ \\
\hline \multicolumn{3}{c}{} \\
$f_{k k^{\prime}}=\frac{\sum_{m=1}^{M} \gamma_{m k} \beta_{m k^{\prime}}}{\left(\sum_{m=1}^{M} \gamma_{m k}\right)^{2}}$, \\
$r_{k}=\frac{(33 \mathrm{~b})}{\rho\left(\sum_{m=1}^{M} \gamma_{m k}\right.}$ \\
\end{tabular}

The transformation in (32) demonstrates that the left-hand side of (32) is a posynomial function. Hence, Problem $P_{11}$ is a standard GP, which completes the proof of Proposition 2.

Based on Proposition 2, the objective function and constraints of Problem $P_{11}$ are monomial and posynomials functions in terms of power allocaitons $\eta_{k}$ s. Hence, Problem $P_{11}$ is a standard GP, and can be efficiently solved through convex optimization software. Simulation results are provided to show that both bisection and GP based sub-optimal schemes achieve the same user rate for all users.

\section{COMPLEXITY ANALYSIS}

Here, we provide the computational complexity analysis for the proposed Algorithm 1, which solves a generalized eigenvalue problem $P_{2}$ and a GP (convex optimization problem) $P_{4}$ at each iteration. For the receiver filter coefficient design in $P_{2}$, given by (13), an eigenvalue solver requires approximately $\mathcal{O}\left(K M^{3}\right)$ flops [34], [35]. Note that the complexity analysis of an eigenvalue solver takes into account the matrix inversion as well. In addition, a standard GP in Problem $P_{4}$, defined in (15), can be solved with complexity equivalent to $\mathcal{O}\left(K^{\frac{7}{2}}\right)$ [36, Ch. 10]. The proposed sub-optimal scheme in Section VII solves a GP in Problem $P_{11}$, defined in (31), which can be solved with $\mathcal{O}\left(K^{\frac{7}{2}}\right)$ [36, Ch. 10]. However, for the scheme in [4], the iterative bisection search method in Algorithm 2 solves a SOCP at each iteration. The complexity of SOCP is $\mathcal{O}\left(K^{4}\right)$ in each iteration [37], [38]. Note that the total number of iterations to solve Problem $P_{9}$ via a bisection search method is given by $\log _{2}\left(\frac{t_{\max }-t_{\min }}{\epsilon}\right)$, where $\epsilon$ refers to a predetermined threshold [33]. The number of arithmetic operations required for Algorithm 1, Algorithm 2, and the proposed sub-optimal scheme are provided in Table I.

\section{Proposed User Assignment Scheme}

In practice, the total backhaul capacity required between the $m$ th AP and the CPU increases linearly with the total number of users served by the $m$ th AP, which motivates the need to pick a proper set of active users for each AP [28]. In [28], we proposed a user assignment algorithm which can reduce 
the required capacity of backhaul link by assigning a limited number of users to each AP, however, this paper assumes perfect backhaul links. Hence, for simplicity we assume here that only $t_{m} \%$ of the total number of users can be supported by the $m$ th AP. Hence, we have

$$
K_{m} \leq\left(\frac{\mathrm{th}_{m}}{100} \times K\right),
$$

where $K_{m}$ denotes the size of the set of active users for the $m$ th AP. First, we find an upper bound on the size of the set of active users for each AP. In the next step, we propose for all APs that the users are sorted according to $\beta_{m k}, \forall k$, and find the $K_{m}$ users which have the highest values of $\beta_{m k}$ among all users. If a user is not selected by any AP, we propose to find the AP which has the best link to this user. Then, we add the user to the set of active users for this AP and drop the user which has the lowest $\beta_{m k}, \forall k$, among active users for that AP which have links to other APs as well. We next solve the original max-min SINR problem with $\tilde{\gamma}_{m k} \leftarrow \gamma_{m k}$, where $\tilde{\gamma}_{m k}$ is given by

$$
\tilde{\gamma}_{m k}= \begin{cases}\gamma_{m k}, & m \in \mathcal{S}_{k} \\ 0, & \text { otherwise },\end{cases}
$$

where $\mathcal{S}_{k}$ refers to the set of active APs for the $k$ th user. Note that optimum user assignment scheme can be considered in future work.

\section{Numerical Results AND Discussion}

In this section, we provide numerical simulation results to validate the performance of the proposed max-min SINR scheme with different parameters. A cell-free Massive MIMO system with $M$ APs and $K$ single-antenna users is considered in a $D \times D$ simulation area, where both APs and users are uniformly located at random. In the following subsections, we define the simulation parameters and then present the corresponding simulation results.

\section{A. Simulation Parameters}

The channel coefficients between users and APs are modeled in (1) where the coefficient $\beta_{m k}$ is given by [4]

$$
\beta_{m k}=\mathrm{PL}_{m k} 10 \frac{\sigma_{s h} z_{m k}}{10},
$$

where $\mathrm{PL}_{m k}$ is the path loss from the $k$ th user to the $m$ th AP and the second term in (36), $10 \frac{\frac{\sigma_{s h} z_{m k}}{10}}{10}$, denotes the shadow fading with standard deviation $\sigma_{s h}=8 \mathrm{~dB}$, and $z_{m k} \sim$ $\mathcal{N}(0,1)$. In the simulation, an uncorrelated shadowing model is considered and a three-slope model for the path loss is given by [4], [39]

$$
\mathrm{PL}_{m k}= \begin{cases}-L-35 \log _{10}\left(d_{m k}\right), & d_{m k}>d_{1}, \\ -L-15 \log _{10}\left(d_{1}\right)-20 \log _{10}\left(d_{m k}\right), & d_{0}<d_{m k} \leq d_{1}, \\ -L-15 \log _{10}\left(d_{1}\right)-20 \log _{10}\left(d_{0}\right), & d_{m k} \leq d_{0},\end{cases}
$$

and $L=46.3+33.9 \log _{10}(f)-13.82 \log _{10}\left(h_{A P}\right)-$ $\left(1.1 \log _{10}(f)-0.7\right) h_{k}+\left(1.56 \log _{10}(f)-0.8\right)$, where $f$ denotes the carrier frequency (in $\mathrm{MHz}$ ), $h_{A P}$ and $h_{k}$ represent the AP antenna height (in $\mathrm{m}$ ) and user height (in $\mathrm{m}$ ),

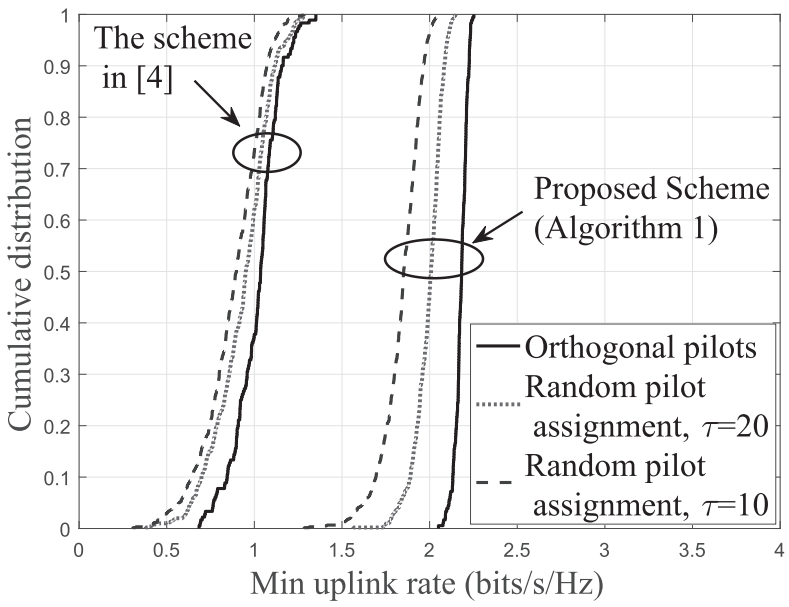

Fig. 2. The cumulative distribution of the min uplink rate, with orthogonal and random pilots for $M=120, K=30$ and $D=1 \mathrm{~km}^{2}$.

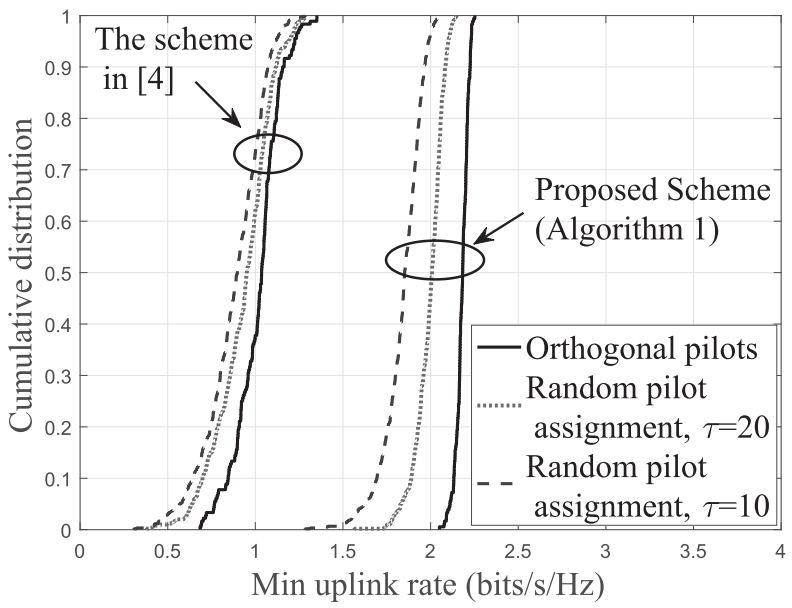

Fig. 3. The cumulative distribution of the min uplink rate, with orthogonal and random pilots for $M=120, K=30$ and $D=1 \mathrm{~km}^{2}$.

respectively. The noise power is given by $p_{n}=\mathrm{BW} \times k_{B} \times$ $T_{0} \times W$, where $\mathrm{BW}=20 \mathrm{MHz}$ denotes the bandwidth, $k_{B}=1.381 \times 10^{-23}$ represents the Boltzmann constant, and $T_{0}=290$ (Kelvin) denotes the noise temperature. Moreover, $W=9 \mathrm{~dB}$, and denotes the noise figure. It is assumed that that $\bar{p}_{p}$ and $\bar{\rho}$ denote the pilot sequence and the uplink data powers, respectively, where $p_{p}=\frac{p_{p}}{p_{n}}$ and $\rho=\frac{\bar{\rho}}{p_{n}}$. In simulations, we set $\bar{p}_{p}=200 \mathrm{~mW}$ and $\bar{\rho}=200 \mathrm{~mW}$. Similar to [4], we assume that the simulation area is wrapped around at the edges which can simulate an area without boundaries. Hence, the square simulation area has eight neighbours. We evaluate the average rate of the system over 300 random realizations of the locations of APs, users and shadow fading. Furthermore, to consider the channel estimation overhead in our comparison, we exploit the net throughput of the system which is defined as [4] $R_{\text {net, } \mathrm{k}}=\mathrm{BW} \frac{1-\frac{\tau}{\tau_{c}}}{2} R_{k}$, where $\tau_{c}$ represents the coherence interval in samples.

\section{B. Simulation Results}

1) Performance of the Proposed Max-Min SINR Algorithm: In this subsection, we evaluate the performance of the proposed uplink max-min SINR scheme. To assess the perfor- 


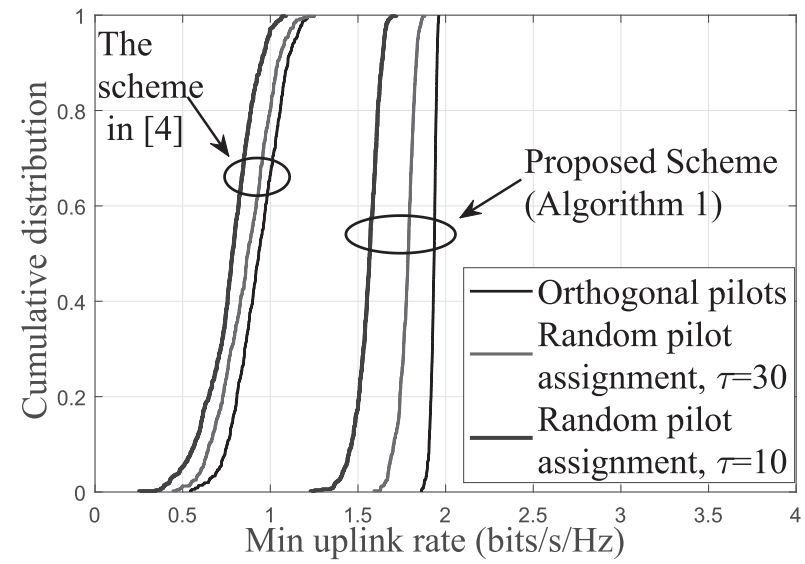

Fig. 4. The cumulative distribution of the min uplink rate, with random pilots for $M=150, K=50$ and $D=1 \mathrm{~km}^{2}$.

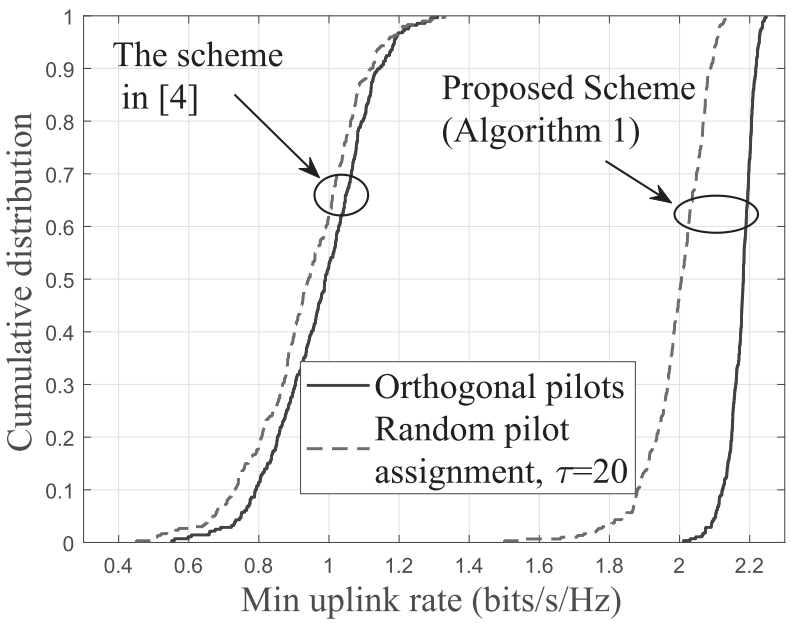

Fig. 5. The cumulative distribution of the min uplink rate with proposed user assignment scheme in Section IX, with orthogonal and random pilots for $M=120, K=30, K_{m}=20, \forall m$, and $D=1 \mathrm{~km}^{2}$. mance, a cell-free Massive MIMO system is considered with 120 APs $(M=120)$ and 30 users $(K=30)$ who are randomly distributed over the simulation area of size $1 \times 1 \mathrm{~km}^{2}$. Fig. 3 presents the cumulative distribution of the achievable uplink rates for the proposed Algorithm 1 and the scheme in [4], for the cases of orthogonal and random pilots. As seen in Fig. 3, the performance of the proposed scheme is almost three times than that of the scheme in [4]. Next, the performance of the algorithm is evaluated for a system with 150 APs $(M=150)$ and 50 users $(K=50) .{ }^{1}$ Fig. 4 similarly compares the rate of the proposed algorithm with the scheme in [4]. The simulation results in Figs. 3 and 4 show that the proposed Algorithm 1 achieves more than double the $10 \%$ outage capacity compared to the scheme in [4]. Moreover, Figs. 3 and 4 demonstrate that the rate of the proposed max-min SINR approach is more concentrated around the median value.

2) User Assignment: In this subsection, the performance of the proposed uplink max-min SINR scheme with the proposed user assignment scheme in Section IX is investigated. We set

${ }^{1}$ The analysis in [40] demonstrates that in the limit of Massive MIMO $\left(M, K \rightarrow \infty\right.$ and $\left.\alpha=\frac{M}{K}\right)$, when $\alpha \geq 4$, linear precoding is "virtually optimal", and can be used instead of dirty paper coding (DPC). In this paper, we consider the two cases $\alpha=\frac{120}{30}=4$ and $\alpha=\frac{150}{50}=3$.

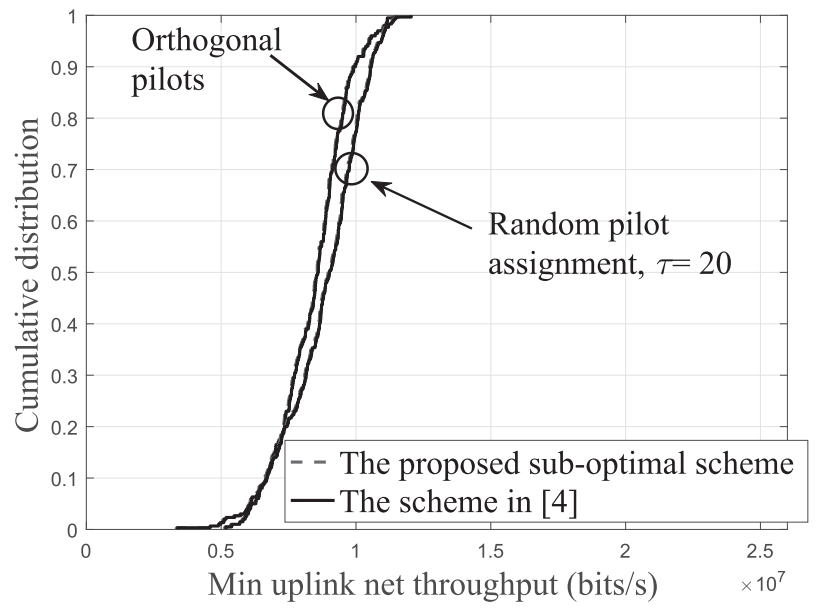

Fig. 6. The cumulative distribution of the min uplink net throughput, with orthogonal and random pilots for $M=120, K=30, D=1 \mathrm{~km}^{2}$ and $\tau_{c}=200$.

120 APs $(M=120)$ and 30 users $(K=30)$, and assume $66.66 \%$ of the total number of users can be supported by each AP. Based on the analysis in Section IX, this results in a total number of users supported users by each AP of $K_{m}=20, \forall m$. Fig. 5 presents the cumulative distribution of the achievable uplink rates for the proposed Algorithm 1 and the scheme in [4] with the proposed user assignment algorithm in Section IX, for the cases of orthogonal and random pilots. As seen in Fig. 5, the performance of the proposed scheme is significantly better than that of the scheme in [4]. In addition, it can be observed from figure that the rate of the proposed Algorithm 1 is more concentrated around the median. Interestingly, by comparing the results in Figs. 3 and 5, the performance degradation is negligible exploiting the proposed user assignment scheme whereas based on the analysis in [28], the backhaul rate is significantly reduced.

3) Performance of the Proposed Sub-Optimal Scheme: In this subsection, we study the effect of the proposed suboptimal scheme on the system performance. Fig. 6 compares the cumulative distribution of the achievable uplink net throughput for our proposed sub-optimal scheme with scheme in [4]. In order to generate the numerical results for the scheme in [4], the iterative bisection search method in Algorithm 2 is used whereas the proposed sub-optimal scheme solves the standard GP with polynomial time complexity. In Fig. 6, the same cell-free Massive MIMO system is considered with 120 APs $(M=120)$ and 30 users $(K=30)$. Figs. 6 and 7 compare the performance of the proposed sub-optimal approach with the scheme in [4] for different system parameters. As evidenced from these numerical results, both proposed GP approach and the bisection search scheme in [4] shows the same performance in terms of the achieved user rate. However, the scheme in [4] is developed through iterative bisection search in which a SOCP is solved at each iteration, whereas the proposed GP approach does not require any iterative methods and solves the problem with polynomial time complexity.

4) Convergence: Next, we provide simulation results to validate the convergence of the proposed algorithm for a set 


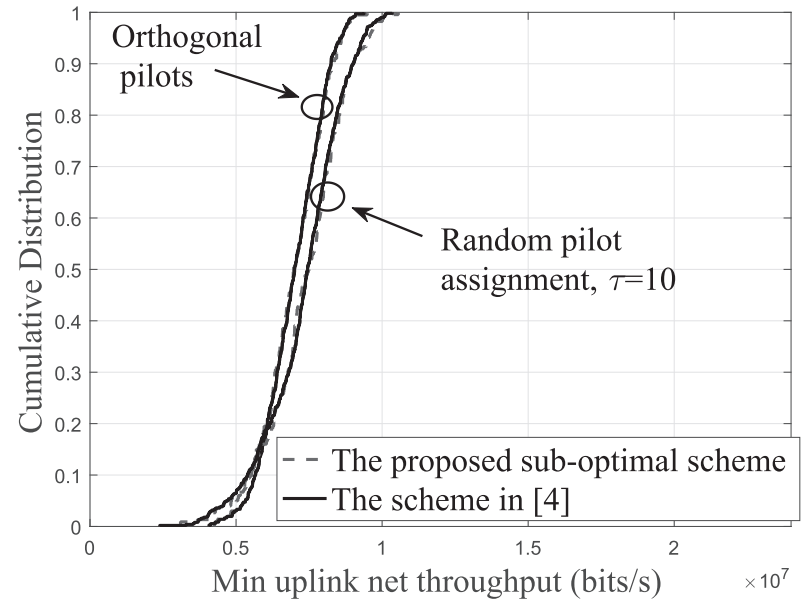

Fig. 7. The cumulative distribution of the min uplink net throughput, with orthogonal and random pilots for $M=150, K=50, D=1 \mathrm{~km}^{2}$ and $\tau_{c}=200$.

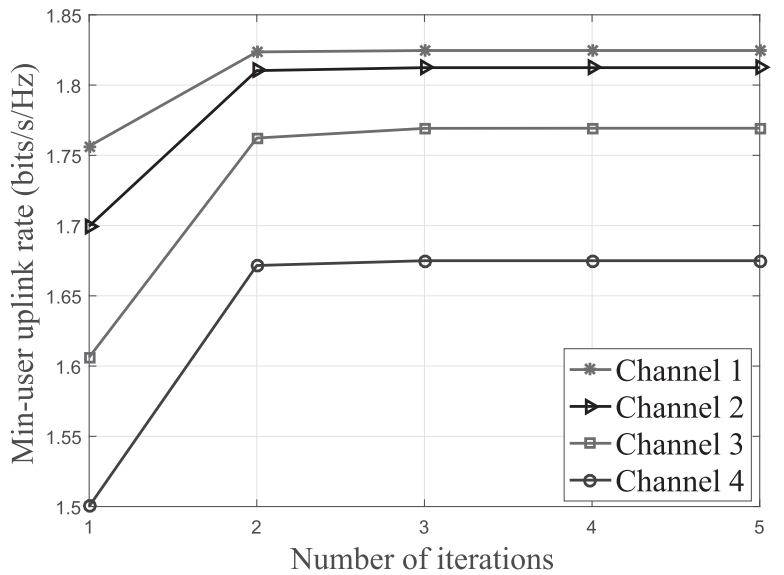

Fig. 8. The convergence of the proposed max-min SINR approach (Algorithm 1) for $M=120, K=30$ and $D=1 \mathrm{~km}$ with orthogonal pilots.

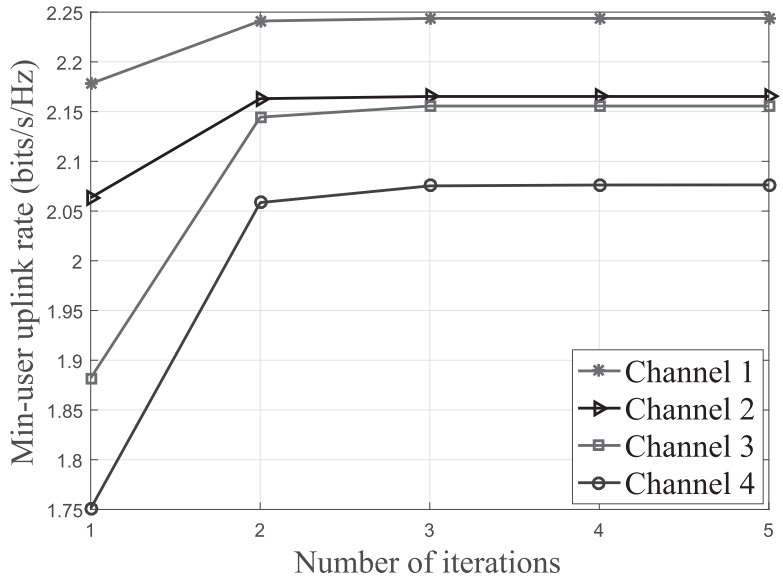

Fig. 9. The convergence of the proposed max-min SINR approach (Algorithm 1) for $M=150, K=50, D=1 \mathrm{~km}$, and the length of the pilot sequences is set to $30(\tau=30)$.

of different channel realizations. These results are generated over the simulation area of size $1 \times 1 \mathrm{~km}^{2}$ with random and orthogonal pilot sequences. Fig. 8 investigates the convergence of the proposed Algorithm 1 with 120 APs $(M=120)$

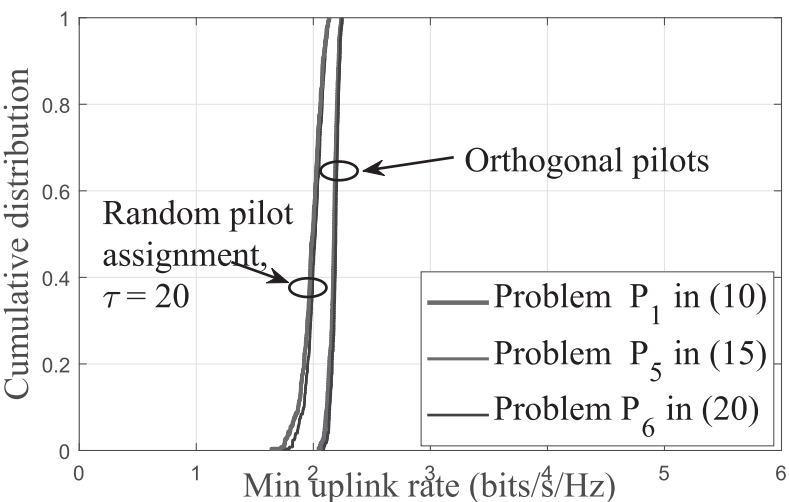

Fig. 10. The cumulative distribution of the min uplink rate for the original problem with per-user power constraint (Problem $P_{1}$ ), the equivalent uplink problem with total power constraint (Problem $P_{5}$ ), and the equivalent downlink problem (Problem $P_{6}$ ), with orthogonal and random pilots for $M=120, K=30$ and $D=1 \mathrm{~km}$

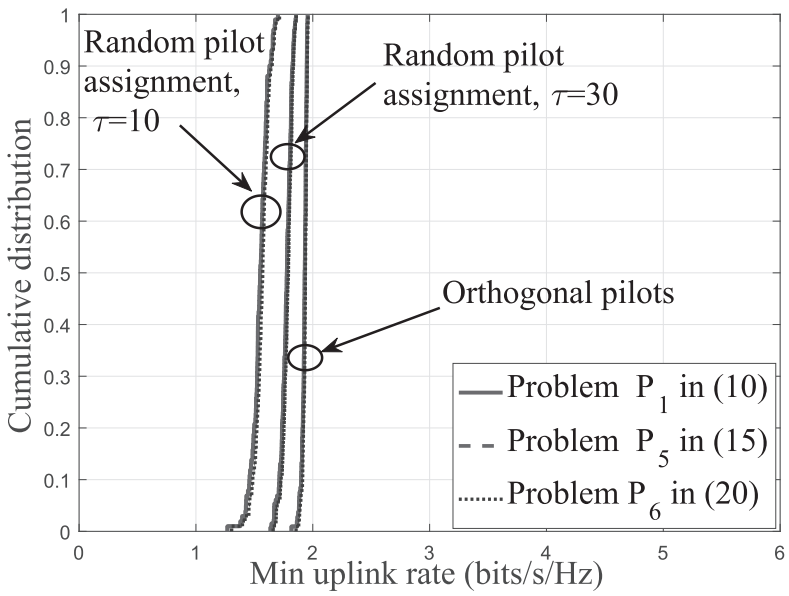

Fig. 11. The cumulative distribution of the min uplink rate for the original problem with per-user power constraint (Problem $P_{1}$ ), the equivalent uplink problem with total power constraint (Problem $P_{5}$ ), and the equivalent downlink problem (Problem $P_{6}$ ), with orthogonal and random pilots for $M=150, K=50$ and $D=1 \mathrm{~km}$.

and 30 users $(K=30)$ and orthogonal pilot sequences, whereas Fig. 9 demonstrates the convergence of the proposed Algorithm 1 for the case of $M=150$ APs and $K=50$. The figures confirm that the proposed algorithm converges after a few iterations, while the minimum rate of the users increases with the iteration number.

5) Uplink-Downlink Duality in Cell-Free Massive MIMO System: Here, the simulation results are provided to support the theoretical derivations of the uplink-downlink duality and the optimality of Algorithm 1. It is assumed that users are randomly distributed through the simulation area of size $1 \times 1 \mathrm{~km}^{2}$. Figs. 10 and 11 compare the cumulative distribution of the achievable uplink rates between the original uplink max-min problem (Problem $P_{1}$ ), the equivalent uplink problem (Problem $P_{5}$ ) and the equivalent downlink problem (Problem $P_{6}$ ). In Fig. 10, the minimum uplink rate is obtained for a system with 120 APs $(M=120)$ and 30 users $(K=30)$ whereas Fig. 11 presents the same results for 150 APs $(M=150)$ and 50 users $(K=50)$. The simulation results provided in Figs. 10 and 11 validate our result that 
the problem formulations $P_{1}, P_{5}$ and $P_{6}$ are equivalent and achieve the same minimum user rate. In addition, these results support our result on the uplink-downlink duality for cell-free Massive MIMO in Section VI and the proof of optimality of Algorithm 1.

\section{Conclusions}

We have considered cell-free Massive MIMO which has the potential to meet the capacity requirements of $5 \mathrm{G}$. Compared to the collocated Massive MIMO, the distributed version brings the APs much closer to the "cell edge" users, which leads to a uniformly good service for all users. We have investigated the uplink max-min SINR problem in cell-free Massive MIMO systems and proposed an optimal solution to maximize the minimum uplink user rate. To realize the solution, the original max-min problem was divided into two sub-problems which were iteratively solved by formulating them respectively as a generalized eigenvalue problem and as GP. The optimality of the proposed solution has been validated by establishing the uplink-downlink duality for cellfree Massive MIMO systems. Next, a novel sub-optimal scheme was developed through formulating the max-min power allocation problem as a standard GP, which efficiently and globally solves the max-min SINR problem. Simulation results have been provided to demonstrate the effectiveness and the optimality of the proposed schemes in comparison with the existing schemes. In addition, these results confirm that the proposed max-min SINR algorithm can significantly improve the uplink user rate, compared to existing algorithms.

\section{APPENDIX A}

\section{ProOF OF THEOREM 1}

The desired signal for user $k$ is given by

$\mathrm{DS}_{k}=\sqrt{\rho \mathbb{E}}\left\{\sum_{m=1}^{M} u_{m k} \hat{g}_{m k}^{*} g_{m k} \sqrt{q_{k}}\right\}=\sqrt{\rho q_{k}} \sum_{m=1}^{M} u_{m k} \gamma_{m k}$.

Hence,

$$
\left|\mathrm{DS}_{k}\right|^{2}=\rho q_{k}\left(\sum_{m=1}^{M} u_{m k} \gamma_{m k}\right)^{2} .
$$

Moreover, the term $\mathbb{E}\left\{\left|\mathrm{BU}_{k}\right|^{2}\right\}$ can be obtained as

$$
\begin{aligned}
\mathbb{E}\left\{\left|\mathrm{BU}_{k}\right|^{2}\right\} & \\
=\rho \mathbb{E} & \left\{\mid \sum_{m=1}^{M} u_{m k} \hat{g}_{m k}^{*} g_{m k} \sqrt{q_{k}}\right. \\
& \left.-\left.\rho \mathbb{E}\left\{\sum_{m=1}^{M} u_{m k} \hat{g}_{m k}^{*} g_{m k} \sqrt{q_{k}}\right\}\right|^{2}\right\} \\
= & \rho \sum_{m=1}^{M} q_{k} u_{m k}^{2}\left(\mathbb{E}\left\{\left|\hat{g}_{m k}^{*} g_{m k}-\mathbb{E}\left\{\hat{g}_{m k}^{*} g_{m k}\right\}\right|^{2}\right\}\right) \\
= & \rho q_{k} \sum_{m=1}^{M} u_{m k}^{2} \gamma_{m k} \beta_{m k},
\end{aligned}
$$

where the last equality comes from the analysis in 835 [4, Appendix A], and using the following fact that; $\gamma_{m k}=$ $\mathbb{E}\left\{\left|\hat{g}_{m k}\right|^{2}\right\}=\sqrt{\tau p_{p}} \beta_{m k} c_{m k}$. The term $\mathbb{E}\left\{\left|\mathrm{IUI}_{k k^{\prime}}\right|^{2}\right\}$ is derived as

$$
\begin{aligned}
\mathbb{E}\left\{\left|\mathrm{IUI}_{k k^{\prime}}\right|^{2}\right\} & \rho \mathbb{E}\left\{\left|\sum_{m=1}^{M} u_{m k} \hat{g}_{m k}^{*} g_{m k^{\prime}} \sqrt{q_{k^{\prime}}}\right|^{2}\right\} \\
= & p \mathbb{E}\left\{\mid \sum_{m=1}^{M} c_{m k} u_{m k} g_{m k^{\prime}} \sqrt{q_{k^{\prime}}}\right. \\
\times & \left.\times\left.\left(\sqrt{\tau p_{p}} \sum_{i=1}^{K} g_{m i} \boldsymbol{\phi}_{k}^{H} \boldsymbol{\phi}_{i}+\boldsymbol{\phi}_{k}^{H} \mathbf{n}_{p, m}\right)^{*}\right|^{2}\right\} \\
= & \underbrace{q_{k^{\prime}} \mathbb{E}\left\{\left|\sum_{m=1}^{M} c_{m k} u_{m k} g_{m k^{\prime}} \tilde{n}_{m k}^{*}\right|^{2}\right\}}_{B} \underbrace{}_{A} \underbrace{\rho \tau p_{p} \mathbb{E}\left\{q_{k^{\prime}}\left|\sum_{m=1}^{M} c_{m k} u_{m k} g_{m k^{\prime}}\left(\sum_{i=1}^{K} g_{m i} \boldsymbol{\phi}_{k}^{H} \boldsymbol{\phi}_{i}\right)^{*}\right|^{2}\right\}},
\end{aligned}
$$

where the third equality in (41) is due to the fact that for two independent random variables $X$ and $Y$ and $\mathbb{E}\{X\}=$ 0 , we have $\mathbb{E}\left\{|X+Y|^{2}\right\}=\mathbb{E}\left\{|X|^{2}\right\}+\mathbb{E}\left\{|Y|^{2}\right\}$ [4]. Since $\tilde{n}_{m k}=\boldsymbol{\phi}_{k}^{H} \mathbf{n}_{p, m} \sim \mathcal{C N}(0,1)$ is independent of the term $g_{m k^{\prime}}$, the term $A$ in (41) is given immediately by

$$
A=q_{k^{\prime}} \sum_{m=1}^{M} c_{m k}^{2} u_{m k}^{2} \beta_{m k^{\prime}} .
$$

The term $B$ in (41) can be obtained as

$$
\begin{aligned}
B & =\underbrace{\tau p_{p} q_{k^{\prime}} \mathbb{E}\left\{\left.\left.\left|\sum_{m=1}^{M} c_{m k} u_{m k}\right| g_{m k^{\prime}}\right|^{2} \boldsymbol{\phi}_{k}^{H} \boldsymbol{\phi}_{k^{\prime}}\right|^{2}\right\}}_{C} \\
& +\underbrace{\tau p_{p} q_{k^{\prime}} \mathbb{E}\left\{\left|\sum_{m=1}^{M} c_{m k} u_{m k} g_{m k^{\prime}}\left(\sum_{i \neq k^{\prime}}^{K} g_{m i} \boldsymbol{\phi}_{k}^{H} \boldsymbol{\phi}_{i}\right)^{*}\right|^{2}\right\}}_{D} .
\end{aligned}
$$

The first term in (43) is given by

$$
\begin{aligned}
C= & \tau p_{p} q_{k^{\prime}} \mathbb{E}\left\{\left.\left.\left|\sum_{m=1}^{M} c_{m k} u_{m k}\right| g_{m k^{\prime}}\right|^{2} \boldsymbol{\phi}_{k}^{H} \boldsymbol{\phi}_{k^{\prime}}\right|^{2}\right\} \\
= & 2 \tau p_{p} q_{k^{\prime}}\left|\boldsymbol{\phi}_{k}^{H} \boldsymbol{\phi}_{k^{\prime}}\right|^{2} \sum_{m=1}^{M} c_{m k}^{2} u_{m k}^{2} \beta_{m k^{\prime}}^{2}+\tau p_{p} q_{k^{\prime}} \\
& \mathbb{E}\left\{\left|\boldsymbol{\phi}_{k}^{H} \boldsymbol{\phi}_{k^{\prime}}\right|^{2} \sum_{m=1}^{M} \sum_{n \neq m}^{M} c_{m k} c_{n k} u_{m k} u_{n k}\left|g_{m k^{\prime}}\right|^{2}\left|g_{n k^{\prime}}\right|^{2}\right\} \\
= & \tau p_{p} q_{k^{\prime}}\left|\boldsymbol{\phi}_{k}^{H} \boldsymbol{\phi}_{k^{\prime}}\right|^{2} \sum_{m=1}^{M} c_{m k}^{2} u_{m k}^{2} \beta_{m k^{\prime}}^{2} \\
+ & q_{k^{\prime}}\left|\boldsymbol{\phi}_{k}^{H} \boldsymbol{\phi}_{k^{\prime}}\right|^{2}\left(\sum_{m=1}^{M} u_{m k} \gamma_{m k} \frac{\beta_{m k^{\prime}}}{\beta_{m k}}\right)^{2},
\end{aligned}
$$


where the last equality is derived based on the fact $\gamma_{m k}=$ $\sqrt{\tau p_{p}} \beta_{m k} c_{m k}$. The second term in (43) can be obtained as

$$
\begin{aligned}
D & =\tau p_{p} q_{k^{\prime}} \mathbb{E}\left\{\left|\sum_{m=1}^{M} c_{m k} u_{m k} g_{m k^{\prime}}\left(\sum_{i \neq k^{\prime}}^{K} g_{m i} \boldsymbol{\phi}_{k}^{H} \boldsymbol{\phi}_{i}\right)^{*}\right|^{2}\right\} \\
& =\tau p_{p} \sum_{m=1}^{M} \sum_{i \neq k^{\prime}}^{K} q_{k^{\prime}} c_{m k}^{2} u_{m k}^{2} \beta_{m k^{\prime}} \beta_{m i}\left|\boldsymbol{\phi}_{k}^{H} \boldsymbol{\phi}_{i}\right|^{2} .
\end{aligned}
$$

Hence, (41) can be written as

$$
\begin{aligned}
\mathbb{E}\left\{\left|\mathrm{IUI}_{k k^{\prime}}\right|^{2}\right\}= & \underbrace{q_{k^{\prime}} \sum_{m=1}^{M} c_{m k}^{2} u_{m k}^{2} \beta_{m k^{\prime}}}_{C_{1}} \\
& +\tau p_{p} q_{k^{\prime}}\left|\boldsymbol{\phi}_{k}^{H} \boldsymbol{\phi}_{k^{\prime}}\right|^{2} \sum_{m=1}^{M} c_{m k}^{2} u_{m k}^{2} \beta_{m k^{\prime}}^{2} \\
& +\tau p_{p} q_{k^{\prime}} \sum_{m=1}^{M} \sum_{i \neq k^{\prime}}^{K} c_{m k}^{2} u_{m k}^{2} \beta_{m k^{\prime}} \beta_{m i}\left|\boldsymbol{\phi}_{k}^{H} \boldsymbol{\phi}_{i}\right|^{2} \\
& q_{k^{\prime}}\left|\boldsymbol{\phi}_{k}^{H} \boldsymbol{\phi}_{k^{\prime}}\right|^{2}\left(\sum_{m=1}^{M} u_{m k} \gamma_{m k} \frac{\beta_{m k^{\prime}}}{\beta_{m k}}\right)^{2},
\end{aligned}
$$

and

$$
\begin{aligned}
C_{2}= & \tau p_{p} q_{k^{\prime}}\left|\boldsymbol{\phi}_{k}^{H} \boldsymbol{\phi}_{k^{\prime}}\right|^{2} \sum_{m=1}^{M} c_{m k}^{2} u_{m k}^{2} \beta_{m k^{\prime}}^{2} \\
& +\underbrace{\tau p_{p} q_{k^{\prime}} \sum_{m=1}^{M} \sum_{i \neq k^{\prime}}^{M} c_{m k}^{2} u_{m k}^{2} \beta_{m k^{\prime}} \beta_{m i}\left|\boldsymbol{\phi}_{k}^{H} \boldsymbol{\phi}_{i}\right|^{2}}_{C_{3}} .
\end{aligned}
$$

For the last term of (47), we have

$$
\begin{aligned}
& C_{3}=\tau p_{p} q_{k^{\prime}} \sum_{m=1}^{M} \sum_{i \neq k^{\prime}}^{K} c_{m k}^{2} u_{m k}^{2} \beta_{m k^{\prime}} \beta_{m i}\left|\phi_{k}^{H} \boldsymbol{\phi}_{i}\right|^{2} \\
&= \tau p_{p} q_{k^{\prime}}\left(\sum_{m=1}^{M} u_{m k}^{2} c_{m k} \beta_{m k^{\prime}} \sum_{i=1}^{K} c_{m k} \beta_{m i}\left|\boldsymbol{\phi}_{k}^{H} \boldsymbol{\phi}_{i}\right|^{2}\right. \\
&\left.\quad-\sum_{m=1}^{M} u_{m k}^{2} c_{m k}^{2} \beta_{m k^{\prime}}\left|\boldsymbol{\phi}_{k}^{H} \boldsymbol{\phi}_{k^{\prime}}\right|^{2}\right) \\
&=\sqrt{\tau p_{p}} q_{k^{\prime}} \sum_{m=1}^{M} u_{m k}^{2} c_{m k} \beta_{m k^{\prime}} \beta_{m k}-q_{k^{\prime}} \sum_{m=1}^{M} u_{m k}^{2} c_{m k}^{2} \beta_{m k^{\prime}} \\
&-\tau p_{p} q_{k^{\prime}} \sum_{m=1}^{M} u_{m k}^{2} c_{m k}^{2} \beta_{m k^{\prime}}\left|\boldsymbol{\phi}_{k}^{H} \boldsymbol{\phi}_{k^{\prime}}\right|^{2},
\end{aligned}
$$

where in the last step, we used equation (4). As a result, $C_{1}+C_{2}=\sqrt{\tau p_{p}} q_{k^{\prime}} \sum_{m=1}^{M} u_{m k}^{2} c_{m k} \beta_{m k^{\prime}} \beta_{m k}$. Then finally we have

$$
\begin{aligned}
\mathbb{E}\left\{\left|\mathrm{IUI}_{k k^{\prime}}\right|^{2}\right\}= & \rho q_{k^{\prime}}\left(\sum_{m=1}^{M} u_{m k}^{2} \beta_{m k^{\prime}} \gamma_{m k}\right) \\
& +\rho q_{k^{\prime}}\left|\boldsymbol{\phi}_{k}^{H} \boldsymbol{\phi}_{k^{\prime}}\right|^{2}\left(\sum_{m=1}^{M} u_{m k} \gamma_{m k} \frac{\beta_{m k^{\prime}}}{\beta_{m k}}\right)^{2} .
\end{aligned}
$$

The total noise for user $k$ is given by

$$
\mathbb{E}\left\{\left|\mathrm{TN}_{k}\right|^{2}\right\}=\mathbb{E}\left\{\left|\sum_{m=1}^{M} u_{m k} \hat{g}_{m k}^{*} n_{m}\right|^{2}\right\}=\sum_{m=1}^{M} u_{m k}^{2} \gamma_{m k},
$$

where the last equality is due to the fact that the terms $\hat{g}_{m k}$ and $n_{m}$ are uncorrelated. Finally, by substituting (39), (40), (49) and (50) into (9), SINR of $k$ th user is obtained by (10). which completes the proof of Theorem 1.

\section{APPENDIX B}

PROOF OF PROPOSITION 1

The standard form of GP is defined as follows [33]:

$P_{12}: \min f_{0}(\mathbf{x})$,

s.t. $f_{i}(\mathbf{x}) \leq 1, i=1, \cdots, m, g_{i}(\mathbf{x})=1, i=1, \cdots, p$,

where $f_{0}$ and $f_{i}$ are posynomial and $g_{i}$ are monomial functions. Moreover, $\mathbf{x}=\left\{x_{1}, \cdots, x_{n}\right\}$ represent the optimization variables. The SINR constraint in (15c) is not a posynomial function in its form, however it can be rewritten into the following posynomial function:

$$
\begin{array}{r}
\mathbf{u}_{k}^{H}\left(\sum_{k^{\prime} \neq k}^{K} q_{k^{\prime}}\left|\boldsymbol{\phi}_{k}^{H} \boldsymbol{\phi}_{k^{\prime}}\right|^{2} \boldsymbol{\Delta}_{k k^{\prime}} \boldsymbol{\Delta}_{k k^{\prime}}^{H}+\sum_{k^{\prime}=1}^{K} q_{k^{\prime}} \mathbf{D}_{k k^{\prime}}+\frac{1}{\rho} \mathbf{R}_{k}\right) \mathbf{u}_{k} \\
\mathbf{u}_{k}^{H}\left(q_{k} \boldsymbol{\Gamma}_{k} \boldsymbol{\Gamma}_{k}^{H}\right) \mathbf{u}_{k} \\
<\frac{1}{t}, \forall k .
\end{array}
$$

By applying a simple transformation, (52) is equivalent to the following inequality:

$$
q_{k}^{-1}\left(\sum_{k^{\prime} \neq k}^{K} a_{k k^{\prime}} q_{k^{\prime}}+\sum_{k^{\prime}=1}^{K} b_{k k^{\prime}} q_{k^{\prime}}+c_{k}\right)<\frac{1}{t},
$$

where

$$
\begin{aligned}
a_{k k^{\prime}} & =\frac{\mathbf{u}_{k}^{H}\left(\left|\boldsymbol{\phi}_{k}^{H} \boldsymbol{\phi}_{k^{\prime}}\right|^{2} \boldsymbol{\Delta}_{k k^{\prime}} \boldsymbol{\Delta}_{k k^{\prime}}^{H}\right) \mathbf{u}_{k}}{\mathbf{u}_{k}^{H}\left(\boldsymbol{\Gamma}_{k} \boldsymbol{\Gamma}_{k}^{H}\right) \mathbf{u}_{k}}, \\
b_{k k^{\prime}} & =\frac{\mathbf{u}_{k}^{H} \mathbf{D}_{k k^{\prime}} \mathbf{u}_{k}}{\mathbf{u}_{k}^{H}\left(\boldsymbol{\Gamma}_{k} \boldsymbol{\Gamma}_{k}^{H}\right) \mathbf{u}_{k}}, c_{k}=\frac{\mathbf{u}_{k}^{H} \mathbf{R}_{k} \mathbf{u}_{k}}{\rho \mathbf{u}_{k}^{H}\left(\boldsymbol{\Gamma}_{k} \boldsymbol{\Gamma}_{k}^{H}\right) \mathbf{u}_{k}} .
\end{aligned}
$$

The transformation in (53) shows that the left-hand side of (52) is a posynomial function. Therefore, the power allocation problem $P_{4}$ is a standard GP (convex problem), where the objective function and constraints are monomial and posynomial, respectively, which completes the proof of Proposition 1.

\section{APPENDIX C}

\section{PROOF OF LEMMA 1}

This lemma is proven by exploiting the unique optimal solution of uplink max-min SINR problem with total power through an eigensystem [22]. This problem is iteratively solved and the optimal receiver filter coefficient $\tilde{\mathbf{U}}$ is determined by solving Problem $P_{3}$. Next, we scale the power allocation at 


$$
\operatorname{SINR}_{k}^{\mathrm{UP}}=\frac{q_{k} \mathbf{u}_{k}^{H}(\overbrace{\boldsymbol{\Gamma}_{k} \boldsymbol{\Gamma}_{k}^{H}}^{\tilde{\mathbf{D}}_{k}}) \mathbf{u}_{k}}{\mathbf{u}_{k}^{H}(\sum_{k^{\prime} \neq k}^{K} q_{k^{\prime}} \underbrace{\left|\phi_{k}^{H} \boldsymbol{\phi}_{k^{\prime}}\right|^{2} \boldsymbol{\Delta}_{k k^{\prime}} \boldsymbol{\Delta}_{k k^{\prime}}^{H}}_{\tilde{\mathbf{R}}_{k k^{\prime}}}+\sum_{k^{\prime}=1}^{K} q_{k^{\prime}} \underbrace{\mathbf{D}_{k k^{\prime}}}_{\tilde{\tilde{\mathbf{R}}}_{k k^{\prime}}}+\frac{1}{\rho} \mathbf{R}_{k}) \mathbf{u}_{k}} .
$$

each user such that the per-user power constraints are satisfied. Let us consider the following optimization problem for a given receiver filter coefficient $\tilde{\mathbf{U}}$ :

$$
P_{11}: C_{k}^{\mathrm{UP}}\left(\tilde{\mathbf{U}}, P_{\mathrm{tot}}\right)=\max _{q_{k}} \min _{k=1, \cdots, K} \operatorname{SINR}_{k}^{\mathrm{UP}}(\tilde{\mathbf{U}}, \mathbf{q}),
$$

$$
\text { subject to } \sum_{k=1}^{K} q_{k} \leq P_{\text {tot }} \text {. }
$$

The optimal solution of Problem $P_{11}$ can be determined by finding the unique eigenvector of an eigensystem and the power allocation $\tilde{\mathbf{q}}$ satisfies the condition $\sum_{k=1}^{K} \tilde{q}_{k}=P_{\text {tot }}$ [22]. The SINRs of all users defined in (10), can be collectively written as

$$
\tilde{\mathbf{q}} \frac{1}{C_{k}^{\mathrm{UP}}\left(\tilde{\mathbf{U}}, P_{\mathrm{tot}}\right)}=\mathbf{D} \Psi(\tilde{\mathbf{U}}) \tilde{\mathbf{q}}+\mathbf{D} \boldsymbol{\sigma}(\tilde{\mathbf{U}})
$$

where $\boldsymbol{\sigma}(\tilde{\mathbf{U}}) \in \mathbb{C}^{K \times 1}, \sigma_{k}\left(\mathbf{u}_{k}\right)=\frac{1}{\rho} \sum_{m=1}^{M} \tilde{u}_{m k} \gamma_{m k}$ and $\mathbf{D}$ and $\Psi(\tilde{\mathbf{U}})$ are defined as

$$
\begin{array}{r}
\mathbf{D}=\operatorname{diag}\left[\frac{1}{\tilde{\mathbf{u}}_{1}^{H} \tilde{\mathbf{D}}_{1} \tilde{\mathbf{u}}_{1}}, \cdots, \frac{1}{\tilde{\mathbf{u}}_{K}^{H} \tilde{\mathbf{D}}_{K} \tilde{\mathbf{u}}_{K}}\right], \\
{[\Psi(\tilde{\mathbf{U}})]_{k k^{\prime}}=\left\{\begin{array}{l}
\tilde{\mathbf{u}}_{k}^{H} \tilde{\tilde{\mathbf{R}}}_{k k} \tilde{\mathbf{u}}_{k}, \\
\tilde{\mathbf{u}}_{k}^{H} \tilde{\mathbf{R}}_{k k^{\prime}} \tilde{\mathbf{u}}_{k}+\tilde{\mathbf{u}}_{k}^{H} \tilde{\tilde{\mathbf{R}}}_{k k^{\prime}} \tilde{\mathbf{u}}_{k}, k \neq k^{\prime},
\end{array}\right.}
\end{array}
$$

where using (10), $\tilde{\mathbf{D}}_{k} \tilde{\mathbf{R}}_{k k^{\prime}}$ and $\tilde{\tilde{\mathbf{R}}}_{k k^{\prime}}$ are defined as (58), shown at the top of this page.

Having both sides of (56) multiplied by $\mathbf{1}^{T}=[1, \cdots, 1]^{T}$, we obtain $\frac{1}{C_{k}^{\mathrm{UP}}\left(\tilde{\mathbf{U}}, P_{\text {tot }}\right)}=\frac{1}{P_{\text {tot }}} \mathbf{1}^{T} \tilde{\mathbf{D}} \Psi(\tilde{\mathbf{U}}) \tilde{\mathbf{q}}+\frac{1}{P_{\text {tot }}} \mathbf{1}^{T} \mathbf{D} \boldsymbol{\sigma}(\tilde{\mathbf{U}})$, which can be combined with (56) to define the following eigensystem:

$$
\boldsymbol{\Lambda}\left(\tilde{\mathbf{U}}, P_{\mathrm{tot}}\right) \tilde{\mathbf{q}}_{\mathrm{ext}}=\frac{1}{C_{k}^{\mathrm{UP}}\left(\tilde{\mathbf{U}}, P_{\mathrm{tot}}\right)} \tilde{\mathbf{q}}_{\mathrm{ext}}, \quad\left[\tilde{\mathbf{q}}_{\mathrm{ext}}\right]_{K+1}=1,
$$

where the extended coupling matrix $\boldsymbol{\Lambda}\left(\tilde{\mathbf{D}}, P_{\text {tot }}\right)$ is given by

$$
\boldsymbol{\Lambda}\left(\tilde{\mathbf{D}}, P_{\mathrm{tot}}\right)=\left[\begin{array}{cc}
\mathbf{D} \boldsymbol{\Psi}^{T}(\tilde{\mathbf{U}}) & \mathbf{D} \boldsymbol{\sigma}(\tilde{\mathbf{U}}) \\
\frac{1}{P_{\mathrm{tot}}} \mathbf{1}^{T} \mathbf{D} \Psi^{T}(\tilde{\mathbf{U}}) & \frac{1}{P_{\mathrm{tot}}} \mathbf{1}^{T} \mathbf{D} \boldsymbol{\sigma}(\tilde{\mathbf{U}})
\end{array}\right] .
$$

The optimal power allocation $\tilde{\mathbf{q}}$ is obtained by determining the eigenvector corresponding to the maximum eigenvalue of $\Lambda\left(\tilde{\mathbf{U}}, P_{\text {tot }}\right)$ and scaling the last element to one as follows:

$$
\tilde{\mathbf{q}}_{\mathrm{ext}}=\left[\begin{array}{c}
\tilde{\mathbf{q}} \\
1
\end{array}\right], \boldsymbol{\Lambda}\left(\tilde{\mathbf{U}}, P_{\mathrm{tot}}\right) \tilde{\mathbf{q}}_{\mathrm{ext}}=\lambda_{\max }\left(\boldsymbol{\Lambda}\left(\tilde{\mathbf{U}}, P_{\mathrm{tot}}\right)\right) \tilde{\mathbf{q}}_{\mathrm{ext}}
$$

Note that the dominant eigenvector can be scaled by any positive value to satisfy a particular condition. As such, we further scale $\tilde{\mathbf{q}}$ to satisfy the per-user power constraints as follows:

$$
\tilde{\mathbf{q}}=\left[\begin{array}{c}
\frac{\hat{q}_{1}}{\max (\hat{\mathbf{q}})} \\
\vdots \\
\frac{\hat{q}_{K}}{\max (\hat{\mathbf{q}})}
\end{array}\right], \text { where } \hat{\mathbf{q}}=\left[\begin{array}{c}
\tilde{q}_{1}^{(1)} \\
p_{\max }^{(1)} \\
\vdots \\
\tilde{q}_{K} \\
p_{\max }^{(K)}
\end{array}\right],
$$

where first the ratios between each component of the allocated power, $\tilde{q}_{k}, \forall k$, and the maximum available power, $p_{\max }^{(k)}, \forall k$, are calculated. Then the power allocation $\tilde{\mathbf{q}}$ is obtained by dividing all components of $\tilde{\mathbf{q}}$ by the maximum value among the components of $\hat{\mathbf{q}}$, i.e., $\max (\hat{\mathbf{q}})$. In the next iteration, the same max-min problem is solved with a new total power constraint obtained by summing up the allocated power to all users in the previous iteration, i.e., $P_{\text {tot }}=\sum_{k=1}^{K} \tilde{q}_{k}$. At the convergence, the per-user power constraints are satisfied with achieving the same uplink SINR for all users. Interestingly, if this max-min problem is solved with the corresponding total power constraint, then it will converge to the same optimal solution of max-min problem with per-user power constraints. This is due to the property that the eigensystem exploited to obtain the power allocation in (58) has a unique positive eigenvalue and a corresponding unique eigenvector. Therefore, Problems $P_{1}$ and $P_{5}$ are equivalent and have the same optimal solution.

\section{APPENDIX D \\ PROOF OF THEOREM 3}

To achieve the same SINR tuples in both the uplink and the downlink, the following condition should be satisfied:

$$
\operatorname{SINR}_{k}^{\mathrm{DL}}(\mathbf{U}, \mathbf{p})=\operatorname{SINR}_{k}^{\mathrm{UP}}(\mathbf{U}, \mathbf{q}), \forall k .
$$

By substituting uplink and downlink SINRs, in (19) and (18), respectively, in equation (62) and summing all equations by both sides, we have

$$
p_{1} \sum_{m=1}^{M} u_{m 1}^{2} \gamma_{m 1}+\cdots+p_{K} \sum_{m=1}^{M} u_{m K}^{2} \gamma_{m K}=\sum_{k=1}^{K} q_{k} .
$$

Therefore, this condition between the total transmit power on the uplink and the equivalent total transmit power on the downlink should be satisfied to realize the same SINRs for all set of users, which completes the proof of Theorem 3.

\section{REFERENCES}

[1] F. Boccardi, R. W. Heath, A. Lozano, T. L. Marzetta, and P. Popovski, "Five disruptive technology directions for 5G," IEEE Commun. Mag., vol. 52, no. 2, pp. 74-80, Feb. 2014 
[2] A. Zappone, L. Sanguinetti, G. Bacci, E. Jorswieck, and M. Debbah, "Energy-efficient power control: A look at 5G wireless technologies," IEEE Trans. Signal Process., vol. 64, no. 7, pp. 1668-1683, Apr. 2016.

[3] E. Björnson, E. A. Jorswieck, M. Debbah, and B. Ottersten, "Multiobjective signal processing optimization: The way to balance conflicting metrics in 5G systems," IEEE Signal Process. Mag., vol. 31, no. 6, pp. 14-23, Nov. 2014.

[4] H. Q. Ngo, A. Ashikhmin, H. Yang, E. G. Larsson, and T. L. Marzetta, "Cell-free massive MIMO versus small cells," IEEE Trans. Wireless Commun., vol. 16, no. 3, pp. 1834-1850, Mar. 2017.

[5] M. Bashar, K. Cumanan, A. G. Burr, M. Debbah, and H. Q. Ngo, "Enhanced max-min SINR for uplink cell-free massive MIMO systems," in Proc. IEEE ICC, May 2018, pp. 1-7.

[6] E. Nayebi, A. Ashikhmin, T. L. Marzetta, H. Yang, and B. D. Rao, "Precoding and power optimization in cell-free massive MIMO systems," IEEE Trans. Wireless Commun., vol. 16, no. 7, pp. 4445-4459, Jul. 2017.

[7] G. Interdonato, H. Q. Ngo, E. G. Larsson, and P. Frenger, "On the performance of cell-free massive MIMO with short-term power constraints," in Proc. IEEE CAMAD, Oct. 2016, pp. 1-6.

[8] S. Buzzi and C. D'Andrea, "Cell-free massive MIMO: User-centric approach," IEEE Wireless Commun. Lett., vol. 6, no. 6, pp. 706-709, Dec. 2017.

[9] M. K. Karakayali, G. J. Foschini, and R. A. Valenzuela, "Network coordination for spectrally efficient communications in cellular systems," IEEE Wireless Commun., vol. 13, no. 4, pp. 56-61, Aug. 2006.

[10] E. Björnson, R. Zakhour, D. Gesbert, and B. Ottersten, "Cooperative multicell precoding: Rate region characterization and distributed strategies with instantaneous and statistical CSI," IEEE Trans. Signal Process., vol. 58, no. 8, pp. 4298-4310, Aug. 2010.

[11] S.-H. Park, O. Simeone, O. Sahin, and S. Shamai (Shitz), "Fronthaul compression for cloud radio access networks: Signal processing advances inspired by network information theory," IEEE Signal Process. Mag., vol. 31, no. 6, pp. 69-79, Nov. 2014.

[12] S.-H. Park, O. Simeone, and S. Shamai (Shitz), "Joint optimization of cloud and edge processing for fog radio access networks," IEEE Trans. Wireless Commun., vol. 15, no. 11, pp. 7621-7632, Nov. 2016.

[13] H. Q. Ngo, L.-N. Tran, T. Q. Duong, M. Matthaiou, and E. G. Larsson, "On the total energy efficiency of cell-free massive MIMO," IEEE Trans. Green Commun. Netw., vol. 2, no. 1, pp. 25-39, Mar. 2017.

[14] K. Cumanan, R. Krishna, L. Musavian, and S. Lambotharan, "Joint beamforming and user maximization techniques for cognitive radio networks based on branch and bound method," IEEE Trans. Wireless Commun., vol. 9, no. 10, pp. 3082-3092, Oct. 2010.

[15] K. Cumanan, L. Musavian, S. Lambotharan, and A. B. Gershman, "SINR balancing technique for downlink beamforming in cognitive radio networks," IEEE Signal Process. Lett., vol. 17, no. 2, pp. 133-136, Feb. 2010 .

[16] K. Cumanan, J. Tang, and S. Lambotharan, "Rate balancing based linear transceiver design for multiuser MIMO system with multiple linear transmit covariance constraints," in Proc. IEEE ICC, Jun. 2011, pp. 1-5.

[17] A. Wiesel, Y. C. Eldar, and S. Shamai (Shitz), "Linear precoding via conic optimization for fixed MIMO receivers," IEEE Trans. Signal Process., vol. 54, no. 1, pp. 161-176, Jan. 2006.

[18] D. W. H. Cai, T. Q. S. Quek, and C. W. Tan, "A unified analysis of maxmin weighted SINR for MIMO downlink system," IEEE Trans. Signal Process., vol. 59, no. 8, pp. 3850-3862, Aug. 2011.

[19] G. Golub and C. F. Van Loan, Matrix Computations, 2nd ed. Baltimore, MD, USA: The Johns Hopkins Univ. Press, 1996.

[20] S. P. Boyd, S.-J. Kim, A. Hassibi, and L. Vandenberghe, "A tutorial on geometric programming," Optim. Eng., vol. 8, no. 1, pp. 67-128, Apr. 2007.

[21] M. Chiang, C. W. Tan, D. P. Palomar, D. O'Neill, and D. Julian, "Power control by geometric programming," in Resource Allocation in Next Generation Wireless Networks. W. Li and Y. Pan, Eds. Commack, NY, USA: Nova, 2006.

[22] M. Schubert and H. Boche, "Solution of the multiuser downlink beamforming problem with individual SINR constraints," IEEE Trans. Veh. Technol., vol. 53, no. 1, pp. 18-28, Jan. 2004.

[23] M. Schubert and H. Boche, "Iterative multiuser uplink and downlink beamforming under SINR constraints," IEEE Trans. Signal Process., vol. 53, no. 7, pp. 2324-2334, Jul. 2005.

[24] D. N. C. Tse and P. Viswanath, "Downlink-uplink duality and effective bandwidths," in Proc. IEEE ISIT, Jun./Jul. 2002, p. 52.
[25] M. Chiang, P. Hande, T. Lan, and C. W. Tan, "Power control in wireless cellular networks," in Foundation and Trends in Networking. Boston, MA, USA: Now, 2007.

[26] H. Ahmadi, A. Farhang, N. Marchetti, and A. MacKenzie, "A game theoretic approach for pilot contamination avoidance in massive MIMO," IEEE Wireless Commun. Lett., vol. 5, no. 1, pp. 12-15, Feb. 2016.

[27] M. A. Imran, S. A. R. Zaidi, and Z. Shakir, Access, Fronthaul and Backhaul Networks for $5 G$ \& Beyond. Edison, NJ, USA: Institution of Engineering and Technology, 2017.

[28] M. Bashar, K. Cumanan, A. G. Burr, H. Q. Ngo, and M. Debbah, "Cell-free massive MIMO with limited backhaul," in Proc. IEEE ICC, May 2018, pp. 1-7.

[29] M. Bashar, K. Cumanan, A. G. Burr, H. Q. Ngo, and M. Debbah, "Maxmin SINR of cell-free Massive MIMO uplink with optimal uniform quantization," IEEE Trans. Commun., submitted for publication.

[30] A. Burr, M. Bashar, and D. Maryopi, "Cooperative access networks: Optimum fronthaul quantization in distributed massive MIMO and cloud RAN," in Proc. IEEE VTC, Jun. 2018, pp. 1-7.

[31] A. J. Fehske, P. Marsch, and G. P. Fettweis, "Bit per Joule efficiency of cooperating base stations in cellular networks," in Proc. IEEE GLOBECOM Workshops (GC Wkshps), Dec. 2010, pp. 1406-1411.

[32] F. Rashid-Farrokhi, K. J. R. Liu, and L. Tassiulas, "Transmit beamforming and power control for cellular wireless systems," IEEE J. Sel. Areas Commun., vol. 16, no. 8, pp. 1437-1450, Oct. 1998.

[33] S. Boyd and L. Vandenberghe, Convex Optimization. Cambridge, U.K.: Cambridge Univ. Press, 2004.

[34] C.-C. Chien, H.-J. Su, and H.-J. Li, "Joint beamforming and power allocation for MIMO relay broadcast channel with individual SINR constraints," IEEE Trans. Veh. Technol., vol. 63, no. 4, pp. 1660-1677, May 2004.

[35] G. H. Golub and C. F. Van Loan, Matrix Computations, 3rd ed. Baltimore, MD, USA: The Johns Hopkins Univ. Press, 1996.

[36] Y. Nesterov and A. Nemirovskii, Interior-Point Polynomial Algorithms in Convex Programming (Studies in Applied Mathematics). Philadelphia, PA, USA: SIAM, 1994

[37] M. S. Lobo, L. Vandenberghe, S. Boyd, and H. Lebret, "Applications of second-order cone programming," Linear Algebra Appl., vol. 284 nos. 1-3, pp. 193-228, Nov. 1998.

[38] J. F. Sturm, "Using SeDuMi 1.02, a MATLAB toolbox for optimization over symmetric cones," Optim. Methods Softw., vol. 11, nos. 1-4, pp. 625-653, 1999.

[39] A. Tang, J. Sun, and K. Gong, "Mobile propagation loss with a low base station antenna for NLOS street microcells in urban area," in Proc. IEEE VTC, May 2001, pp. 333-336.

[40] F. Rusek et al., "Scaling up MIMO: Opportunities and challenges with very large arrays," IEEE Signal Process. Mag., vol. 30, no. 1, pp. 40-60, Jan. 2013.

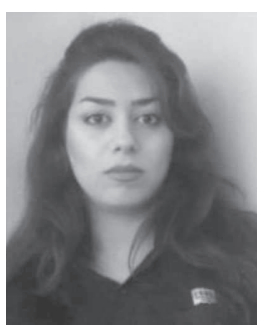

Manijeh Bashar received the B.Sc. degree in electrical engineering from the University of Guilan, Rasht, Iran, in 2009, and the M.Sc. degree in communication systems engineering from the Shiraz University of Technology, Shiraz, Iran, in 2013. She is currently pursuing the Ph.D. degree with the University of York, U.K. She is currently a Research Fellow with the Fifth Generation (5G) Innovation Centre, University of Surrey, U.K.

In 2017, she was an Academic Visitor with the Department of Electronics and Nanoengineering, Aalto University, Espoo, Finland. Her current research interests include cooperative communications for $5 \mathrm{G}$ networks, including distributed massive multiple input multiple output (MIMO), Cloud-RAN, Fog-RAN, nonorthogonal multiple access, network slicing, convex optimization techniques, and millimeter-wave channel modeling. She received the Short Term Scientific Mission Scholarship Award from European COST-IC1004 "Cooperative Radio Communications for Green Smart Environments." She received the First Place (based on jury) at the IEEE WCNC 2018 3-min Ph.D. Thesis Competition for her research in cell-free massive MIMO. 


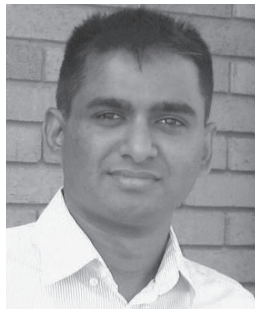

Kanapathippillai Cumanan received the B.Sc degree (Hons.) in electrical and electronic engineering from the University of Peradeniya, Sri Lanka, in 2006, and the Ph.D. degree in signal processing for wireless communications from Loughborough University, Loughborough, U.K., in 2009.

In 2006, he was a Teaching Assistant with the Department of Electrical and Electronic Engineering, University of Peradeniya. In 2011, he was an Academic Visitor with the Department of Electrical and Computer Engineering, National University of Singapore, Singapore. He was with the School of Electronic, Electrical and System Engineering, Loughborough University. From 2012 to 2014, he was a Research Associate with the School of Electrical and Electronic Engineering, Newcastle University, U.K. He is currently a Lecturer with the Department of Electronic Engineering, University of York, U.K. His research interests include non-orthogonal multiple access, massive multiple input multiple output, physical-layer security, cognitive radio networks, convex optimization techniques, and resource allocation techniques.

Between 2006 and 2007, he was a Research Student with Cardiff University, U.K. He received the Overseas Research Student Award Scheme from Cardiff University.

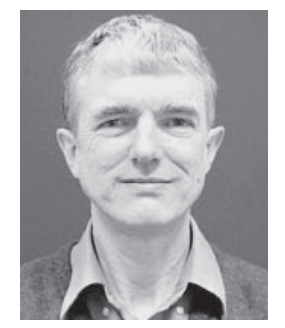

Alister G. Burr was born in London, U.K., in 1957. He received the B.Sc. degree in electronic engineering from The University of Southampton, Southampton, U.K., in 1979, and the Ph.D. degree from the University of Bristol, Bristol, U.K., in 1984. Between 1975 and 1985, he was with the Thorn-EMI Central Research Laboratories, London. In 1985, he joined the Department of Electronics (currently the Department of Electronic Engineering), University of York, York, U.K., where he has been a Professor of communications since 2000. He has authored or co-authored around 250 papers in refereed international conferences and journals. He has authored the book entitled Modulation and Coding for Wireless Communications (Englewood Cliffs, NJ, USA: PrenticeHall/ PHEI, 2001), and has co-authored the book entitled Wireless PhysicalLayer Network Coding (Cambridge, U.K.: Cambridge Univ. Press, 2018). His research interests include wireless communication systems, especially multiple input multiple output, cooperative systems, physical-layer network coding, and iterative detection and decoding techniques. He received the Senior Research Fellowship by the U.K. Royal Society in 1999 and the J. Langham Thompson Premium from the Institution of Electrical Engineers in 2002. He has also given more than 15 invited presentations, including three keynote presentations. He was the Chair, working group 2, of a series of European COST Programs, including IC1004 "Cooperative Radio Communications for Green Smart Environments" (which have been influential in 3GPP standardization). He was an Associate Editor of IEEE COMMUNICATIONS LETTERS, the Workshop Chair of the 2016 IEEE International Conference on Communications, and the Technical Program Committee Co-Chair of the 2018 Annual IEEE International Symposium on Personal, Indoor and Mobile Radio Communications.

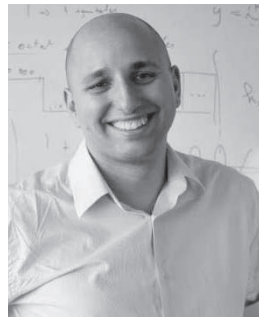

Mérouane Debbah received the M.Sc. and $\mathrm{Ph} . \mathrm{D}$. degrees from the Ecole Normale Supérieure Paris-Saclay, France. In 1996, he joined the Ecole Normale Supérieure Paris-Saclay. He was with Motorola Labs, Saclay, France, from 1999 to 2002, and also with the Vienna Research Center for Telecommunications, Vienna, Austria, until 2003. From 2003 to 2007, he was an Assistant Professor with the Mobile Communications Department, Institut Eurecom, Sophia Antipolis, France. From 2007 to 2014, he was the Director of the Alcatel-Lucent Chair on Flexible Radio. Since 2007, he has been a Full Professor with CentraleSupelec, Gif-sur-Yvette, France. Since 2014, he has been a Vice-President of the Huawei France Research and Development Center and the Director of the Mathematical and Algorithmic Sciences Lab. He has managed eight EU projects and more than 24 national and international projects. His research interests lie in fundamental mathematics, algorithms, statistics, information, and communication sciences research. He is an IEEE Fellow, a WWRF Fellow, and a Membre émérite SEE. He was a recipient of the ERC Grant MORE (Advanced Mathematical Tools for Complex Network Engineering). He was a recipient of the Mario Boella Award in 2005, the IEEE Glavieux Prize Award in 2011, and the Qualcomm Innovation Prize Award in 2012. He received 19 best paper awards, among which the 2007 IEEE GLOBECOM Best Paper Award, the Wi-Opt 2009 Best Paper Award, the 2010 Newcom++ Best Paper Award, the WUN CogCom Best Paper 2012 and 2013 Award, the 2014 WCNC Best Paper Award, the 2015 ICC Best Paper Award, the 2015 IEEE Communications Society Leonard G. Abraham Prize, the 2015 IEEE Communications Society Fred W. Ellersick Prize, the 2016 IEEE Communications Society Best Tutorial Paper Award, the 2016 European Wireless Best Paper Award, the 2017 Eurasip Best Paper Award, the 2018 IEEE Marconi Prize Paper Award, and the Valuetools 2007, Valuetools 2008, CrownCom 2009, Valuetools 2012, SAM 2014, and 2017 IEEE Sweden VT-COM-IT Joint Chapter best student paper awards. He is an Associate Editor-in-Chief of the journal Random Matrix: Theory and Applications. He was an Associate Area Editor and Senior Area Editor of the IEEE TRANSACTIONS ON SIGNAL PROCESSING from 2011 to 2013 and from 2013 to 2014, respectively.

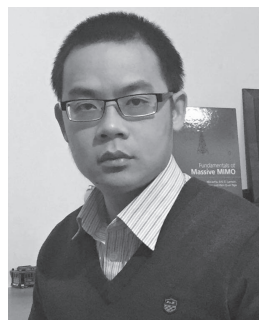

Hien Quoc Ngo received the B.S. degree in electrical engineering from the Ho Chi Minh City University of Technology, Vietnam, in 2007, the M.S. degree in electronics and radio engineering from Kyung Hee University, South Korea, in 2010, and the Ph.D. degree in communication systems from Linköping University (LiU), Sweden, in 2015. In 2014, he visited the Nokia Bell Labs, Murray Hill, NY, USA. From 2016 to 2017, he was a VR Researcher with the Department of Electrical Engineering (ISY), LiU. He was also a Visiting Research Fellow with the School of Electronics, Electrical Engineering and Computer Science, Queen's University Belfast, U.K., funded by the Swedish Research Council.

$\mathrm{He}$ is currently a Lecturer with Queen's University Belfast. He has co-authored many research papers in wireless communications and the textbook Fundamentals of Massive MIMO (Cambridge University Press, 2016). His main research interests include massive (large-scale) multipleinput multiple-output (MIMO) systems, cell-free massive MIMO, physicallayer security, and cooperative communications.

Dr. Ngo was a recipient of the IEEE ComSoc Stephen O. Rice Prize in Communications Theory in 2015, the IEEE ComSoc Leonard G. Abraham Prize in 2017, and the IEEE Sweden VT-COM-IT Joint Chapter Best Student Journal Paper Award in 2015. He received the Best Ph.D. Award from EURASIP in 2018. He was an IEEE Communications Letters Exemplary Reviewer for 2014, an IEEE Transactions on Communications Exemplary Reviewer for 2015, and an IEEE Wireless Communications Letters Exemplary Reviewer for 2016. He currently serves as an Editor for IEEE Wireless Communications Letters, Digital Signal Processing, and the IEICE Transactions on Fundamentals of Electronics, Communication and Computer Sciences. He was a Guest Editor of the IET Communications, special issue on "Recent Advances on 5G Communications" and the IEEE ACCESS, special issue on "Modelling, Analysis, and Design of 5G Ultra-Dense Networks," in 2017. He has been a member of Technical Program Committees for several IEEE conferences, such as ICC, GLOBECOM, WCNC, and VTC. 